\title{
Simplex and Polygon Equations
}

\author{
Aristophanes DIMAKIS ${ }^{\dagger}$ and Folkert MÜLLER-HOISSEN ${ }^{\ddagger}$ \\ $\dagger$ Department of Financial and Management Engineering, University of the Aegean, \\ 82100 Chios, Greece \\ E-mail:dimakis@aegean.gr \\ $\ddagger$ Max Planck Institute for Dynamics and Self-Organization, $3707 r$ Göttingen, Germany \\ E-mail: folkert.mueller-hoissen@ds.mpg.de
}

Received October 23, 2014, in final form May 26, 2015; Published online June 05, 2015

http://dx.doi.org/10.3842/SIGMA.2015.042

\begin{abstract}
It is shown that higher Bruhat orders admit a decomposition into a higher Tamari order, the corresponding dual Tamari order, and a "mixed order". We describe simplex equations (including the Yang-Baxter equation) as realizations of higher Bruhat orders. Correspondingly, a family of "polygon equations" realizes higher Tamari orders. They generalize the well-known pentagon equation. The structure of simplex and polygon equations is visualized in terms of deformations of maximal chains in posets forming 1-skeletons of polyhedra. The decomposition of higher Bruhat orders induces a reduction of the $N$-simplex equation to the $(N+1)$-gon equation, its dual, and a compatibility equation.
\end{abstract}

Key words: higher Bruhat order; higher Tamari order; pentagon equation; simplex equation 2010 Mathematics Subject Classification: 06A06; 06A07; 52Bxx; 82B23

\section{Introduction}

The famous (quantum) Yang-Baxter equation is

$$
\hat{\mathcal{R}}_{12} \hat{\mathcal{R}}_{13} \hat{\mathcal{R}}_{23}=\hat{\mathcal{R}}_{23} \hat{\mathcal{R}}_{13} \hat{\mathcal{R}}_{12},
$$

where $\hat{\mathcal{R}} \in \operatorname{End}(V \otimes V)$, for a vector space $V$, and boldface indices specify the two factors of a threefold tensor product on which $\hat{\mathcal{R}}$ acts. This equation plays an important role in exactly solvable two-dimensional models of statistical mechanics, in the theory of integrable systems, quantum groups, invariants of knots and three-dimensional manifolds, and conformal field theory (see, e.g., [15, 22, 34, 47, 48]).

A set-theoretical version of the Yang-Baxter equation considers $\hat{\mathcal{R}}$ as a map $\hat{\mathcal{R}}: \mathcal{U} \times \mathcal{U} \rightarrow \mathcal{U} \times \mathcal{U}$, where $\mathcal{U}$ is a set (not necessarily supplied with further structure). Nontrivial examples of "settheoretical solutions" of the Yang-Baxter equation, for which Veselov later introduced the name Yang-Baxter maps [98, 99], apparently first appeared in [91] (cf. [99]), and Drinfeld's work [29] stimulated much interest in this subject. Meanwhile quite a number of examples and studies of such maps have appeared (see, e.g., [1, 32, 35, 42, 96]).

The Yang-Baxter equation is a member of a family, called simplex equations [18] (also see, e.g., $[39,68,74,75,77])$. The $N$-simplex equation is an equation imposed on a map $\hat{\mathcal{R}}: V^{\otimes N} \rightarrow$ $V^{\otimes N}$, respectively $\hat{\mathcal{R}}: \mathcal{U}^{N} \rightarrow \mathcal{U}^{N}$ for the set-theoretical version. The next-to-Yang-Baxter equation, the 3 -simplex equation,

$$
\hat{\mathcal{R}}_{123} \hat{\mathcal{R}}_{145} \hat{\mathcal{R}}_{246} \hat{\mathcal{R}}_{356}=\hat{\mathcal{R}}_{356} \hat{\mathcal{R}}_{246} \hat{\mathcal{R}}_{145} \hat{\mathcal{R}}_{123},
$$

is also called tetrahedron equation or Zamolodchikov equation. This equation acts on $V^{\otimes 6}$. A set of tetrahedron equations first appeared as factorization conditions for the $S$-matrix in 
Zamolodchikov's $(2+1)$-dimensional scattering theory of straight lines ("straight strings"), and in a related three-dimensional exactly solvable lattice model [105, 106]. This has been inspired by Baxter's eight-vertex lattice model [11, 12] and stimulated further important work [13, 14], also see the survey [95]. Meanwhile the tetrahedron equation has been the subject of many publications (see, in particular, [20, 21, 43, 59, 68, 70, 76, 89]). An equation of similar structure as the above 3 -simplex equation, but acting on $V^{\otimes 4}$, has been proposed in [39].

In a similar way as the 2 -simplex (Yang-Baxter) equation describes a factorization condition for the scattering matrix of particles in two space-time dimensions [23, 103], as just mentioned, the 3 -simplex equation describes a corresponding condition for straight lines on a plane $[105,106]$. Manin and Schechtman [78, 79] looked for what could play the role of the permutation group, which acts on the particles in the Yang-Baxter case, for the higher simplex equations. They were led in this way to introduce the higher Bruhat order $B(N, n)$, with positive integers $n<N$. This is a partial order on the set of certain equivalence classes of "admissible" permutations of $\left(\begin{array}{c}{[N]} \\ n\end{array}\right)$, which is the set of $n$-element subsets of $[N]:=\{1,2, \ldots, N\}$ (see Section 2.1). The $N$-simplex equation is directly related to the higher Bruhat order $B(N+1, N-1)$.

Let us consider the local Yang-Baxter equation ${ }^{1}$

$$
\hat{\mathcal{L}}_{12}(x) \hat{\mathcal{L}}_{13}(y) \hat{\mathcal{L}}_{\mathbf{2 3}}(z)=\hat{\mathcal{L}}_{\mathbf{2 3}}\left(z^{\prime}\right) \hat{\mathcal{L}}_{\mathbf{1 3}}\left(y^{\prime}\right) \hat{\mathcal{L}}_{\mathbf{1 2}}\left(x^{\prime}\right),
$$

where the $\hat{\mathcal{L}}_{\boldsymbol{i} j}$ depend on variables in such a way that this equation uniquely determines a map $(x, y, z) \mapsto\left(x^{\prime}, y^{\prime}, z^{\prime}\right)$. Then this map turns out to be a set-theoretical solution of the tetrahedron equation. Here we wrote $\hat{\mathcal{L}}_{\boldsymbol{i} \boldsymbol{j}}$ instead of $\hat{\mathcal{R}}_{\boldsymbol{i j}}$ in order to emphasize that such a "localized" equation may be regarded as a "Lax system" for the tetrahedron equation, i.e., the latter arises as a consistency condition of the system. This is a familiar concept in integrable systems theory. If the variables $x, y, z$ are elements of a (finite-dimensional, real or complex) vector space, and if the maps $\hat{\mathcal{L}}_{\boldsymbol{i j}}$ depend linearly on them, then $\hat{\mathcal{L}}_{\boldsymbol{i j}}(x)=x_{a} \hat{\mathcal{L}}_{\boldsymbol{i j}}^{a}$, using the summation convention and expressing $x=x_{a} E^{a}$ in a basis $E^{a}, a=1, \ldots, m$. In this case the above equation takes the form

$$
\hat{\mathcal{L}}_{\mathbf{1 2}}^{a} \hat{\mathcal{L}}_{\mathbf{1 3}}^{b} \hat{\mathcal{L}}_{\mathbf{2 3}}^{c}=\hat{\mathcal{R}}_{\text {def }}^{a b c} \hat{\mathcal{L}}_{\mathbf{2 3}}^{d} \hat{\mathcal{L}}_{\mathbf{1 3}}^{e} \hat{\mathcal{L}}_{\mathbf{1 2}}^{f}
$$

where the coefficients $\hat{\mathcal{R}}_{d e f}^{a b c}$ are defined by $z_{d}^{\prime} y_{e}^{\prime} x_{f}^{\prime}=x_{a} y_{b} z_{c} \hat{\mathcal{R}}_{d e f}^{a b c}$. The last system is also known as the tetrahedral Zamolodchikov algebra (also see $[16,59]$ ). Analogously, there is a Lax system for the Yang-Baxter equation [96], consisting of 1-simplex equations, which is the ZamolodchikovFaddeev algebra [69], and this structure extends to all simplex equations [73, 74, 75, 77]. The underlying idea of relaxing a system of $N$-simplex equations in the above way, by introducing an object $\hat{\mathcal{R}}$, such that consistency imposes the $(N+1)$-simplex equation on it, is the "obstruction method" in $[25,44,73,74,75,77,82]$. Also see $[9,50,51,58,93]$ for a formulation in the setting of 2-categories. Indeed, the obstruction method corresponds to the introduction of laxness ("laxification" [93]).

An equation of a similar nature as the Yang-Baxter equation is the pentagon equation

$$
\hat{\mathcal{T}}_{12} \hat{\mathcal{T}}_{13} \hat{\mathcal{T}}_{23}=\hat{\mathcal{T}}_{23} \hat{\mathcal{T}}_{12}
$$

which appears as the Biedenharn-Elliott identity for Wigner $6 j$-symbols and Racah coefficients in the representation theory of the rotation group [19], as an identity for fusion matrices in conformal field theory [84], as a consistency condition for the associator in quasi-Hopf algebras [27, 28] (also see [3, 4, 10, 33, 36, 40, 41]), as an identity for the Rogers dilogarithm function [87] and matrix generalizations [53], for the quantum dilogarithm [5, 17, 20, 37, 55, 100],

\footnotetext{
${ }^{1}$ In very much the same form, the local Yang-Baxter equation appeared in [88], for example. A natural generalization is obtained by replacing the three appearances of $\hat{\mathcal{L}}$ by three different maps.
} 
and in various other contexts (see, e.g., [26, 52, 56, 57, 60, 67, 72]). In particular, it is satisfied by the Kac-Takesaki operator $(\hat{\mathcal{T}} f)\left(g, g^{\prime}\right)=f\left(g g^{\prime}, g^{\prime}\right), g, g^{\prime} \in G, G$ a group, where it expresses the associativity of the group operation (see, e.g., [101]). A unitary operator acting on $\mathcal{H} \otimes \mathcal{H}$, where $\mathcal{H}$ is a Hilbert space, and satisfying the pentagon equation, has been termed a multiplicative unitary $[6,7,8,46,81,90,101,104]$. It plays an essential role in the development of harmonic analysis on quantum groups. Under certain additional conditions, such an operator can be used to construct a quantum group on the $C^{*}$ algebra level $[6,45,90,101] .^{2}$ For any locally compact quantum group, a multiplicative unitary can be constructed in terms of the coproduct. Any finite-dimensional Hopf algebra is characterized by an invertible solution of the pentagon equation [83]. The pentagon equation arises as a 3-cocycle condition in Lie group cohomology and also in a category-theoretical framework (see, e.g., [94]).

A pentagon relation arises from "laxing" the associativity law [71, 92, 97]. In its most basic form, it describes a partial order (on a set of five elements), which is the simplest Tamari lattice (also see [85]). This is $T(5,3)$ in the notation of this work (also see [25]).

We will show that the pentagon equation belongs to an infinite family of equations, which we call polygon equations. They are associated with higher Tamari orders, as defined ${ }^{3}$ in [25], in very much the same way as the simplex equations are associated with higher Bruhat orders (also see [25]). We believe that these higher Tamari orders coincide with higher Stasheff-Tamari orders, defined in terms of triangulations of cyclic polytopes [30, 49, 86]. ${ }^{4}$

In Section 2.2 we show that any higher Bruhat order can be decomposed into a corresponding higher Tamari order, its dual (which is the reversed Tamari order), and a "mixed order". A certain projection of higher Bruhat to higher (Stasheff-) Tamari orders appeared in [49] (also see [86] and references cited there). Whereas this projects, for example, $B(4,1)$ (permutahedron) to $T(6,3)$ (associahedron), we describe a projection $B(4,1) \rightarrow T(4,1)$ (tetrahedron), and more generally $B(N, n) \rightarrow T(N, n)$.

The $(N+1)$-simplex equation arises as a consistency condition of a system of $N$-simplex equations. The higher Bruhat orders are also crucial for understanding this "integrability" of the simplex equations. In the same way, the higher Tamari orders provide the combinatorial tools to express integrability of polygon equations.

Using the transposition map $\mathcal{P}$ to define $\mathcal{R}:=\hat{\mathcal{R}} \mathcal{P}$, and generalizing this to maps $\mathcal{R}_{i j}: V_{i} \otimes$ $V_{j} \rightarrow V_{j} \otimes V_{i}$, the Yang-Baxter equation takes the form

$$
\mathcal{R}_{23, \mathbf{1}} \mathcal{R}_{13, \mathbf{2}} \mathcal{R}_{12, \mathbf{1}}=\mathcal{R}_{12, \mathbf{2}} \mathcal{R}_{13, \mathbf{1}} \mathcal{R}_{23, \mathbf{2}} \quad \text { on } \quad V_{1} \otimes V_{2} \otimes V_{3}
$$

$\mathcal{R}_{i j, \boldsymbol{a}}:=\mathcal{R}_{i j, \boldsymbol{a}, \boldsymbol{a}+1}$ acts on $V_{i}$ and $V_{j}$, at positions $\boldsymbol{a}$ and $\boldsymbol{a}+1$, in a product of such spaces. The higher Bruhat orders ensure a correct matching of the two different types of indices. In fact, the boldface indices are completely determined, they do not contain independent information. Fig. 1 shows a familiar visualization of the Yang-Baxter equation in terms of deformations of chains of edges on a cube. Supplying the latter with the Bruhat order $B(3,0)$, these are maximal chains. The information given in the caption of Fig. 1 will also be relevant for subsequent figures in this work.

The (weak) Bruhat orders $B(N, 1), N>2$, form polytopes called permutahedra. Not all higher Bruhat orders can be realized on polytopes. The $N$-simplex equation is associated with the Bruhat order $B(N+1, N-1)$, but its structure is rather visible on $B(N+1, N-2)$. The latter possesses a reduction to the 1-skeleton of a polyhedron on which the simplex equation can

\footnotetext{
${ }^{2}$ See [102] for the example of the Hopf algebra of the quantum plane $a b=q^{2} b a$ with $q$ a root of unity.

${ }^{3}$ This definition of higher Tamari orders emerged from our exploration of a special class of line soliton solutions of the Kadomtsev-Petviashvili (KP) equation [24, 25].

${ }^{4}$ More precisely, $\operatorname{HST}_{1}(n, d)$, as defined, e.g., in [86], is expected to be order isomorphic to $T(n, d+1)$, as defined in Section 2.
} 


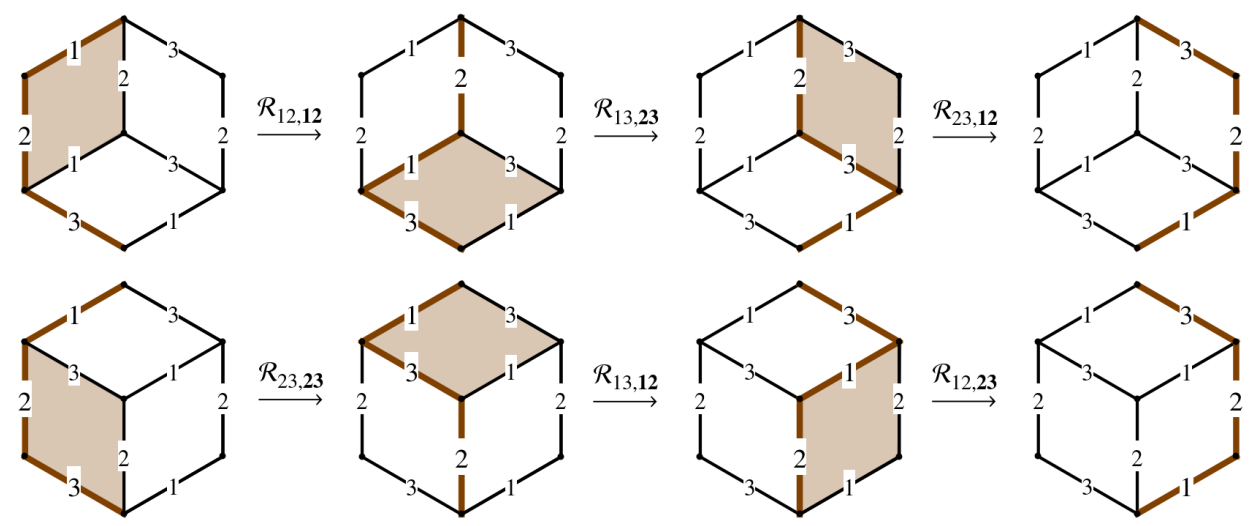

Figure 1. The first row shows a sequence of maximal chains on half of the poset $B(3,0)$, which is the Boolean lattice on $\{1,2,3\}$. The second row shows a corresponding sequence on the complementary part. Glued together along their boundaries, they form a cube. The two ways of deforming the initial lexicographically ordered maximal chain to the final, reverse lexicographically ordered chain, results in a consistency condition, which is the Yang-Baxter equation (1.2). Edges are associated with spaces $V_{i}, i=$ $1,2,3$. For example, the second step in the first row corresponds to the action of id $\otimes \mathcal{R}_{13}$ on $V_{2} \otimes V_{1} \otimes V_{3}$. The boldface indices that determine where, in a product of spaces, the map acts, correspond to the positions of the active edges in the respective (brown) maximal chain, counting from the top downward.

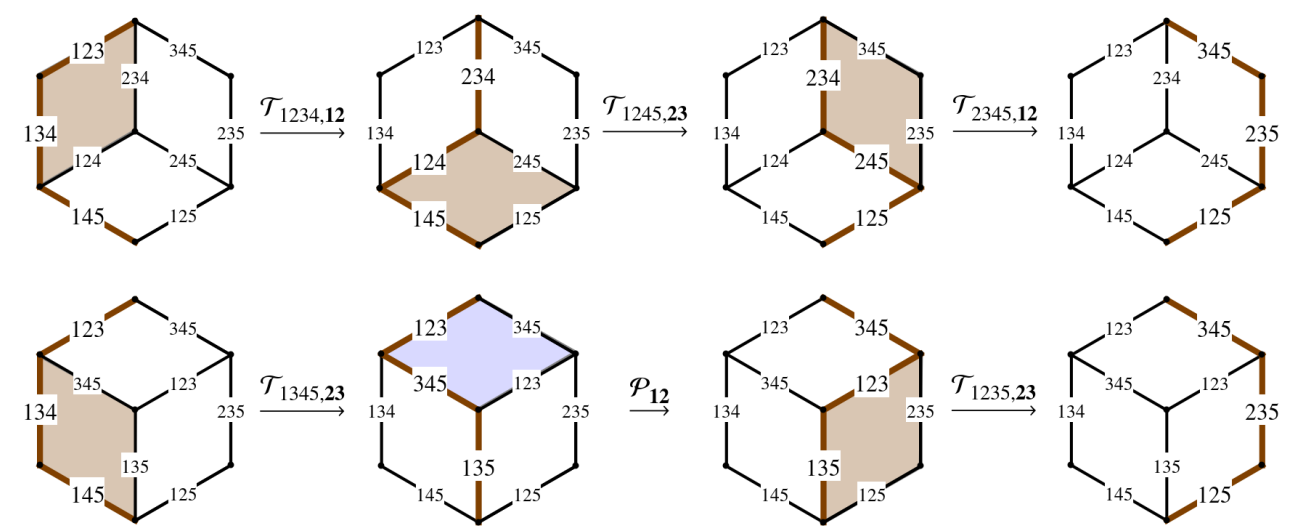

Figure 2. Here the poset is $T(5,2)$, which also forms a cube. Edges are now numbered by 3-element subsets of $12345:=\{1,2,3,4,5\}$. If the edges of a face are labeled by the four 3 -element subsets of $i j k l$, there is a map $\mathcal{T}_{i j k l}$ associated with it. This rule does not apply to the second step in the second row, however, since here the edges of the active face involve five (rather than only four) digits. The two possibilities of deforming the initial (lexicographically ordered) maximal chain $(123,134,145)$ into the final (reverse lexicographically ordered) maximal chain $(345,235,125)$ result in the pentagon equation $(1.3)$.

be visualized in the same way as the Yang-Baxter equation is visualized on $B(3,0)$ (also see [2] for a similar view). This is elaborated in Section 3.

Also for the polygon equations, proposed in this work, all appearances of a map, like $\mathcal{T}$ in the pentagon equation in Fig. 2, will be treated as a priori different maps (now on both sides of the equation). Again we attach to them additional indices that carry combinatorial information, now governed by higher Tamari orders.

The Tamari orders $T(N, 1), N=3,4, \ldots, T(N, 2), N=4,5, \ldots, T(N, 3), N=5,6, \ldots$, as defined in [25] and Section 2, form simplexes, hypercubes and associahedra (Stasheff-Tamari polytopes), respectively. But not all Tamari orders can be realized on polytopes.

The $N$-gon equation is associated with $T(N, N-2)$, but its structure is rather revealed by 
$T(N, N-3)$. For small enough $N$, the latter forms a polyhedron. For higher $N$ it admits a polyhedral reduction. The structure of the $N$-gon equation can then be visualized in terms of deformations of maximal chains on the corresponding polyhedron. Fig. 2 shows the example of the pentagon equation, here obtained in the form

$$
\mathcal{T}_{2345, \mathbf{1}} \mathcal{T}_{1245, \mathbf{2}} \mathcal{T}_{1234, \mathbf{1}}=\mathcal{T}_{1235, \mathbf{2}} \mathcal{P}_{\mathbf{1}} \mathcal{T}_{1345, \mathbf{2}}
$$

(also see Section 4). This implies (1.1) for $\hat{\mathcal{T}}=\mathcal{T} \mathcal{P}$.

Section 2 first provides a brief account of higher Bruhat orders [78, 79, 80, 107]. The main result in this section is a decomposition of higher Bruhat orders, where higher Tamari orders (in the form introduced in [25]) naturally appear. Section 3 explains the relation between higher Bruhat orders and simplex equations, and how the next higher simplex equation arises as a consistency condition of a localized system of simplex equations. Section 4 associates in a similar way polygon equations, which generalize the pentagon equation, with higher Tamari orders. As in the case of simplex equations, the $(N+1)$-gon equation arises as a consistency condition of a system of localized $N$-gon equations. Section 5 reveals relations between simplex and polygon equations, in particular providing a deeper explanation for and considerably generalizing a relation between the pentagon equation and the 4-simplex equation, first observed in [57]. Finally, Section 6 contains some concluding remarks and Appendix A supplements all this by expressing some features of simplex and polygon equations via a more abstract approach.

\section{Higher Bruhat and Tamari orders}

In the first subsection we recall some material about higher Bruhat orders from [78, 79, 80, 107]. The second subsection introduces a decomposition of higher Bruhat orders that includes higher Tamari orders, in the form we defined them in [25].

\subsection{Higher Bruhat orders}

For $N \in \mathbb{N}$, let $[N]$ denote the set $\{1,2, \ldots, N\}$. The packet $P(K)$ of $K \subset[N]$ is the set of all subsets of $K$ of cardinality one less than that of $K$. For $K=\left\{k_{1}, \ldots, k_{n+1}\right\}$ in natural order, i.e., $k_{1}<\cdots<k_{n+1}$, we set

$$
\begin{aligned}
& \vec{P}(K):=\left(K \backslash\left\{k_{n+1}\right\}, K \backslash\left\{k_{n}\right\}, \ldots, K \backslash\left\{k_{1}\right\}\right) \\
& \overleftarrow{P}(K):=\left(K \backslash\left\{k_{1}\right\}, K \backslash\left\{k_{2}\right\}, \ldots, K \backslash\left\{k_{n+1}\right\}\right)
\end{aligned}
$$

The first displays the packet of $K$ in lexicographical order $\left(<_{\text {lex }}\right)$. The second displays $P(K)$ in reverse lexicographical order.

Let $\left(\begin{array}{c}{[N]} \\ n\end{array}\right), 0 \leq n \leq N$, denote the set of all subsets of $[N]$ of cardinality $n$. Its cardinality is

$$
c(N, n):=\left(\begin{array}{c}
N \\
n
\end{array}\right) \text {. }
$$

A linear (or total) order $\rho$ on $\left(\begin{array}{c}{[N]} \\ n\end{array}\right)$ can be written as a sequence $\rho=\left(J_{1}, \ldots, J_{c(N, n)}\right)$ with $J_{a} \in$ $\left(\begin{array}{c}{[N]} \\ n\end{array}\right)$. It is called admissible if, for all $K \in\left(\begin{array}{c}{[N]} \\ n+1\end{array}\right), \rho$ induces on $P(K)$ either the lexicographical or the reverse lexicographical order, i.e., either $\vec{P}(K)$ or $\overleftarrow{P}(K)$ is a subsequence of $\rho$. Let $A(N, n)$ denote the set of admissible linear orders of $\left(\begin{array}{c}{[N]} \\ n\end{array}\right)$.

The envelope $E(J)$ of $J \in\left(\begin{array}{c}{[N]} \\ n\end{array}\right)$ is the set of $K \in\left(\begin{array}{c}{[N]} \\ n+1\end{array}\right)$ such that $J \in P(K)$. An equivalence relation on $A(N, n)$ is obtained by setting $\rho \sim \rho^{\prime}$ if $\rho$ and $\rho^{\prime}$ only differ by a sequence of exchanges of neighboring elements $J, J^{\prime}$ with $E(J) \cap E\left(J^{\prime}\right)=\varnothing$. We set

$$
B(N, n):=A(N, n) / \sim \text {. }
$$


Example 2.1. $\left(\begin{array}{c}{[4]} \\ 2\end{array}\right)=\{12,13,14,23,24,34\}$, where $i j:=\{i, j\}$, allows $6 !=720$ linear orders, but only 14 are admissible. For example, $\rho=(12,34,14,13,24,23) \in A(4,2)$, since it contains the packets of the four elements of $\left(\begin{array}{c}{[4]} \\ 3\end{array}\right)$ in the orders $\vec{P}(123), \vec{P}(124), \overleftarrow{P}(134), \overleftarrow{P}(234)$. We have $\rho \sim(34,12,14,13,24,23) \sim(34,12,14,24,13,23) . B(4,2)=A(4,2) / \sim$ has 8 elements.

The inversion set $\operatorname{inv}[\rho]$ of $\rho \in A(N, n)$ is the set of all $K \in\left(\begin{array}{c}{[N]} \\ n+1\end{array}\right)$ such that $P(K)$ is contained in $\rho$ in reverse lexicographical order. All members of the equivalence class $[\rho] \in B(N, n)$ have the same inversion set. Next we introduce the inversion operation

$$
I_{K}: \vec{P}(K) \mapsto \overleftarrow{P}(K)
$$

If $\vec{P}(K)$ appears in $\rho \in A(N, n)$ at consecutive positions, let $I_{K} \rho$ be the linear order obtained by inversion of $\vec{P}(K)$ in $\rho$. Then ${ }^{5} I_{K} \rho \in A(N, n)$ and $\operatorname{inv}\left[I_{K} \rho\right]=\operatorname{inv}[\rho] \cup\{K\}$. This corresponds to the covering relation

$$
[\rho] \stackrel{K}{\rightarrow}\left[I_{K} \rho\right],
$$

which determines the higher Bruhat order on the set $B(N, n)[78,80]$. In the following, we will mostly drop the adjective "higher". $B(N, n)$ has a unique minimal element $[\alpha]$ that contains the lexicographically ordered set $\left(\begin{array}{c}{[N]} \\ n\end{array}\right)$, hence $\operatorname{inv}[\alpha]=\varnothing$, and a unique maximal element $[\omega]$ that contains the reverse lexicographically ordered set $\left(\begin{array}{c}{[N]} \\ n\end{array}\right)$, hence $\operatorname{inv}[\omega]=\left(\begin{array}{c}{[N]} \\ n+1\end{array}\right)$. The Bruhat orders are naturally extended by defining $B(N, 0)$ as the Boolean lattice on $[N]$, which corresponds to the 1 -skeleton of the $N$-cube, with edges directed from a fixed vertex toward the opposite vertex.

Remark 2.2. There is a natural correspondence between the elements of $A(N, n+1)$ and the maximal chains of $B(N, n)$ [80]. Associated with $\sigma=\left(K_{1}, \ldots, K_{c(N, n+1)}\right) \in A(N, n+1)$ is the maximal chain

$$
[\alpha] \stackrel{K_{1}}{\longrightarrow}\left[\rho_{1}\right] \stackrel{K_{2}}{\longrightarrow}\left[\rho_{2}\right] \stackrel{K_{3}}{\longrightarrow} \cdots \stackrel{K_{c(N, n+1)}}{\longrightarrow}[\omega]
$$

where $\operatorname{inv}\left[\rho_{r}\right]=\left\{K_{1}, \ldots, K_{r}\right\}$. This allows to construct $B(N, n)$ from $B(N, n+1)$. As a consequence, all Bruhat orders $B(N, n), n<N-1$, can be constructed recursively from the highest non-trivial, which is $B(N, N-1)$.

Example 2.3. $B(N, N-1)$ is simply $\vec{P}([N]) \stackrel{[N]}{\rightarrow} \overleftarrow{P}([N])$. From the two admissible linear orders $\vec{P}([N])=(\hat{N}, \ldots, \hat{2}, \hat{1})$ and $\overleftarrow{P}([N])=(\hat{1}, \hat{2}, \ldots, \hat{N})$, where $\hat{k}:=[N] \backslash\{k\}$ ("complementary notation"), we can construct the two maximal chains of $B(N, N-2)$ :

$$
\begin{aligned}
& {[\alpha] \stackrel{\hat{N}}{\longrightarrow}\left[\rho_{1}\right] \stackrel{\widehat{N-1}}{\longrightarrow}\left[\rho_{2}\right] \longrightarrow \cdots \longrightarrow\left[\rho_{N-1}\right] \stackrel{\hat{1}}{\longrightarrow}[\omega],} \\
& {[\alpha] \stackrel{\hat{1}}{\longrightarrow}\left[\sigma_{1}\right] \stackrel{\hat{2}}{\longrightarrow}\left[\sigma_{2}\right] \longrightarrow \cdots \longrightarrow\left[\sigma_{N-1}\right] \stackrel{\hat{N}}{\longrightarrow}[\omega] .}
\end{aligned}
$$

The example $B(4,2)$ is displayed below in $(2.2)$.

Remark 2.4. $U \subset\left(\begin{array}{c}{[N]} \\ n+1\end{array}\right)$ is called a consistent set if, for all $L \in\left(\begin{array}{c}{[N]} \\ n+2\end{array}\right), U \cap P(L)$ can be ordered in such a way that it becomes a beginning segment either of $\vec{P}(L)$ or of $\overleftarrow{P}(L){ }^{6}$ Consistent sets are in bijective correspondence with inversion sets [107].

\footnotetext{
${ }^{5}$ Since two different packets have at most a single member in common, such an inversion does not change the order of other packets in $\rho$ than that of $K$.

${ }^{6} \mathrm{~A}$ beginning segment of a sequence is a subsequence that starts with the first member of the sequence and contains all its members up to a final one. Also the empty sequence and the full sequence are beginning segments.
} 

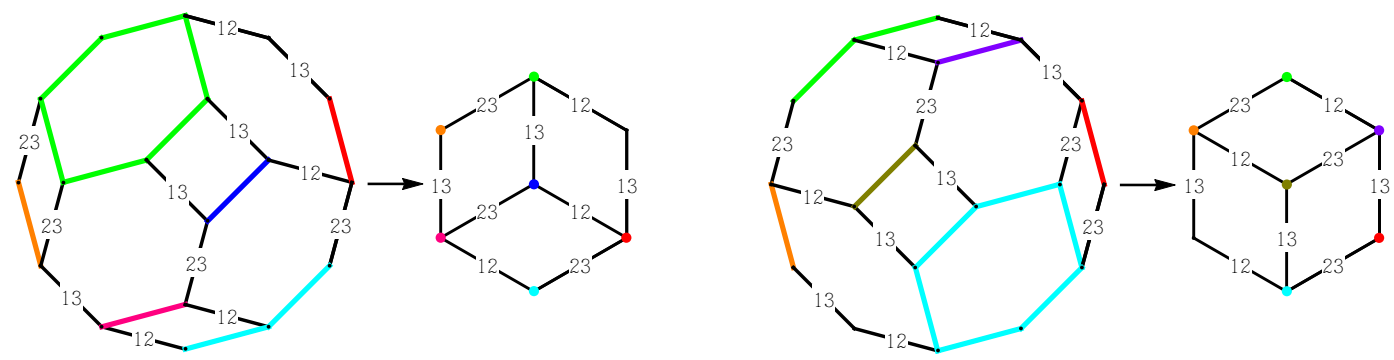

Figure 3. Projection of $B(4,1)$ (permutahedron) to $B(3,0)$ (cube), each split into two complementary parts. Here we chose $k=4$ in Remark 2.5 and use complementary notation for the labels, but with hats omitted. The coloring marks those parts in the two Bruhat orders that are related by the projection.
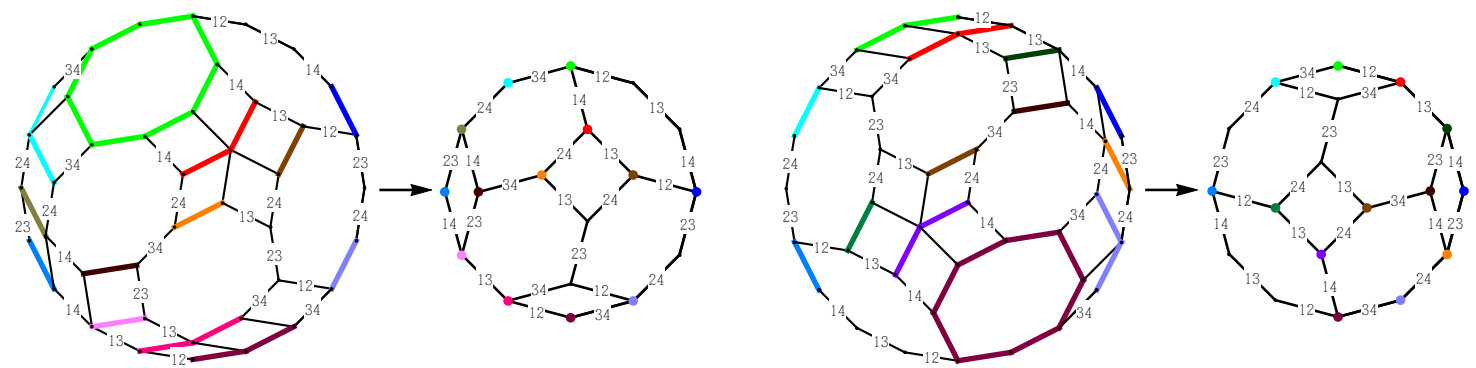

Figure 4. Projection of $B(5,2)$ (Felsner-Ziegler polyhedron [38]) to $B(4,1)$ (permutahedron). Here we chose $k=5$ in Remark 2.5. Again, we use complementary labeling, and the coloring marks the parts related by the projection.

Remark 2.5. For fixed $k \in[N+1]$, we define an equivalence relation in $A(N+1, n+1)$ as follows. Let $\rho \stackrel{k}{\sim} \rho^{\prime}$ if $\rho$ and $\rho^{\prime}$ only differ in the order of elements $K \in\left(\begin{array}{c}{[N+1]} \\ n+1\end{array}\right)$ with $k \notin K$. For $\rho=\left(K_{1}, \ldots, K_{c(N+1, n+1)}\right) \in A(N+1, n+1)$, the equivalence class $\rho_{(k)} \in A(N+1, n+1) / \stackrel{k}{\sim}$ is then completely characterized by the subsequence $\left(K_{i_{1}}, \ldots, K_{i_{c(N, n)}}\right)$ consisting of only those $K_{i}$ that contain $k$. Hence we can identify $\rho_{(k)}$ with this subsequence. As a consequence, there is an obvious bijection between $A(N+1, n+1) \stackrel{k}{\sim}$ and $A(N, n)$. Clearly, inversions of packets $P(L), L \in\left(\begin{array}{c}{[N+1]} \\ n+2\end{array}\right)$, with $k \notin L$, have no effect on the equivalence classes. If $k \in L$, and if $\vec{P}(L)$ appears in $\rho$ at consecutive positions, then $\left(I_{L} \rho\right)_{(k)}$ is obtained from $\rho_{(k)}$ by the inversion $\vec{P}(L) \backslash\{L \backslash\{k\}\} \rightarrow \overleftarrow{P}(L) \backslash\{L \backslash\{k\}\}$. Since the latter naturally corresponds to $\vec{P}(L \backslash\{k\}) \rightarrow \overleftarrow{P}(L \backslash\{k\})$, the bijection $A(N+1, n+1) / \stackrel{k}{\sim} \rightarrow A(N, n)$ is monotone, i.e. order-preserving. Since the equivalence relation $\sim$ is compatible with $\stackrel{k}{\sim}$, the bijection induces a corresponding monotone bijection $B(N+1, n+1) \stackrel{k}{\sim} \rightarrow B(N, n)$. We will use this projection in Section 3.4. Examples are shown in Figs. 3 and 4.

Example 2.6. $\alpha=(123,124,134,234,125,135,235,145,245,345)$ represents the minimal element $[\alpha]$ of $B(5,3)$. Let $k=5$. Then $\alpha_{(5)}$ is represented by $(125,135,235,145,245,345)$. This corresponds to $(12,13,23,14,24,34)$, which represents the minimal element of $B(4,2)$. Inversion of the packet of $L=\{i, j, m, 5\}, 1 \leq i<j<m<5$, corresponds to inversion of the packet of $\{i, j, m\}$, which defines an edge in $B(4,2)$.

\subsection{Three color decomposition of higher Bruhat orders}

For $K \in\left(\begin{array}{c}{[N]} \\ n+1\end{array}\right)$, let $P_{o}(K)$, respectively $P_{e}(K)$, denote the half-packet of elements of $P(K)$ with odd, respectively even, position in the lexicographical order. We assign colors to elements 
of $\vec{P}(K)$, respectively $\overleftarrow{P}(K)$, as follows. An element of $P_{o}(K)$ is blue in $\vec{P}(K)$ and red in $\overleftarrow{P}(K)$, and an element of $P_{e}(K)$ is red in $\vec{P}(K)$ and blue in $\overleftarrow{P}(K)$

Example 2.7. For $K=\{1,2,3,4,5\}=12345$, we have $P_{o}(K)=\{1234,1245,2345\}$ and $P_{e}(K)=\{1235,1345\}$. Hence

$$
\vec{P}(12345)=(1234,1235,1245,1345,2345), \quad \overleftarrow{P}(12345)=(2345,1345,1245,1235,1234)
$$

We say $J \in\left(\begin{array}{c}{[N]} \\ n\end{array}\right)$ is blue (red) in $\rho \in A(N, n)$ if, for all $K \in E(J), J$ is blue (red) in $\vec{P}(K)$, respectively $\overleftarrow{P}(K)$, depending in which order $P(K)$ appears in $\rho .^{7} J$ is called green in $\rho$, if there are $K, K^{\prime} \in E(J)$, such that $J$ is blue with respect to $K$ and red with respect to $K^{\prime}$.

Example 2.8. The following element of $A(5,3)$ has empty inversion set,

$$
\alpha=(123,124,125,134,135,145,234,235,245,345) .
$$

For example, we have $E(124)=\{1234,1245\}$, and $\alpha$ contains $\vec{P}_{o}(1245)=(124,145)$ and $\overrightarrow{P_{e}}(1234)=(124,234)$ as subsequences. This shows that 124 is blue in $\vec{P}(1245)$ and red in $\vec{P}(1234)$, therefore green in $\alpha$.

For each $c \in\{b, r, g\}$ (where $b, r, g$ stands for blue, red and green, respectively), we define an equivalence relation on $A(N, n): \rho \sim_{c} \rho^{\prime}$ if $\rho$ and $\rho^{\prime}$ have the same elements with color $c$ in the same order. Let $\rho^{(c)}$ denote the corresponding equivalence class, and

$$
A^{(c)}(N, n):=A(N, n) / \sim_{c}, \quad c \in\{b, r, g\} .
$$

$\rho^{(c)}$ can be identified with the subsequence of elements in $\rho$ having color $c$.

The definition of the color of an element $J$ of a linear order $\rho$ only involves the inversion set of $\rho$, but not $\rho$ itself (also see footnote 7). Hence, if $J$ has color $c$ in $\rho$, then it has the same color in any element of $[\rho]$. As a consequence, for each $c$, the equivalence relation $\sim_{c}$ is compatible with $\sim$. Defining

$$
B^{(c)}(N, n):=A^{(c)}(N, n) / \sim=(A(N, n) / \sim) / \sim_{c}, \quad c \in\{b, r, g\},
$$

we thus obtain a projection $B(N, n) \rightarrow B^{(c)}(N, n)$ via $[\rho] \mapsto\left[\rho^{(c)}\right]$. We will show that the resulting single-colored sets inherit a partial order from the respective Bruhat order.

Lemma 2.9. Let $K \in E(J) \cap P_{o}(L)$ (respectively, $K \in E(J) \cap P_{e}(L)$ ) for some $J \in\left(\begin{array}{c}{[N]} \\ n\end{array}\right)$ and $L \in\left(\begin{array}{c}{[N]} \\ n+2\end{array}\right)$, where $n<N-1$. Let $K^{\prime} \in E(J) \cap P(L), K^{\prime} \neq K$.

If $K<_{\text {lex }} K^{\prime}$, then $J \in P_{o}\left(K^{\prime}\right)$ (respectively, $J \in P_{e}\left(K^{\prime}\right)$ ).

If $K^{\prime}<_{\operatorname{lex}} K$, then $J \in P_{e}\left(K^{\prime}\right)$ (respectively, $J \in P_{o}\left(K^{\prime}\right)$ ).

Proof. Since $K, K^{\prime} \in E(J)$ and $K \neq K^{\prime}$, we can write $K=J \cup\{k\}$ and $K^{\prime}=J \cup\left\{k^{\prime}\right\}$, with $k, k^{\prime} \notin J, k \neq k^{\prime}$, and $L=K \cup\left\{k^{\prime}\right\}=K^{\prime} \cup\{k\} . K<_{\operatorname{lex}} K^{\prime}$ is equivalent to $k<k^{\prime}$. Let us write $\vec{P}(L)=\left(L \backslash\left\{\ell_{n+2}\right\}, \ldots, L \backslash\left\{\ell_{1}\right\}\right)$ with $\ell_{1}<\ell_{2}<\cdots<\ell_{n+2}$.

$K \in P_{o}(L)\left(K \in P_{e}(L)\right)$ means that $K=L \backslash\left\{k^{\prime}\right\}$ has an odd (even) position in $\vec{P}(L)$, hence $k^{\prime}$ has an odd (even) position in $\left(\ell_{n+2}, \ldots, \ell_{1}\right)$. If $k<k^{\prime}$, then removal of $k$ from $\left(\ell_{n+2}, \ldots, \ell_{1}\right)$ does not change this, so that $k^{\prime}$ also has an odd (even) position in $\left(\ell_{n+2}, \ldots, \check{k}, \ldots, \ell_{1}\right)$, where indicates an omission. It follows that $J=K^{\prime} \backslash\left\{k^{\prime}\right\} \in P_{o}\left(K^{\prime}\right)\left(J \in P_{e}\left(K^{\prime}\right)\right)$. If $k^{\prime}<k$, then the position of $k^{\prime}$ in $\left(\ell_{n+2}, \ldots, \check{k}, \ldots, \ell_{1}\right)$ is even (odd), hence $J=K \backslash\left\{k^{\prime}\right\} \in P_{e}\left(K^{\prime}\right)$ $\left(J \in P_{o}\left(K^{\prime}\right)\right)$.

\footnotetext{
${ }^{7}$ This means that $J$ is blue (red) in $\rho$ if $J \in P_{o}(K)\left(J \in P_{e}(K)\right)$ for all $K \in E(J) \backslash \operatorname{inv}[\rho]$, and $J \in P_{e}(K)$ $\left(J \in P_{o}(K)\right)$ for all $K \in E(J) \cap \operatorname{inv}[\rho]$.
} 
In view of the bijection between $A(N, n+1)$ and the set of maximal chains of $B(N, n)$, it is natural to say that $[\rho] \stackrel{K}{\rightarrow}\left[I_{K} \rho\right]$ has color $c$ in some maximal chain of $B(N, n)$ if $K$ has this color in the associated element of $A(N, n+1)$. An equivalent statement, formulated next, in particular shows that the color of $[\rho] \stackrel{K}{\rightarrow}\left[I_{K} \rho\right]$ is the same in any maximal chain in which it appears, hence we can speak about $[\rho] \stackrel{K}{\rightarrow}\left[I_{K} \rho\right]$ having color $c$ in $B(N, n)$.

If $\vec{P}(L) \cap \operatorname{inv}[\rho]$ is a beginning segment, we will say that $\vec{P}(L)$ has beginning segment with respect to $[\rho]$. A corresponding formulation applies with $\vec{P}(L)$ replaced by $\overleftarrow{P}(L)$. Let $n<N-1$. Then $[\rho] \stackrel{K}{\rightarrow}\left[I_{K} \rho\right]$ is blue (red) if, for all $L \in E(K)$, either $\vec{P}(L)$ has beginning segment w.r.t. [ $\rho$ ] and also w.r.t. $\left[I_{K} \rho\right]$, and $K \in P_{o}(L)\left(K \in P_{e}(L)\right)$, or $\overleftarrow{P}(L)$ has beginning segments w.r.t. [ $\rho$ ] and $\left[I_{K} \rho\right]$, and $K \in P_{e}(L)\left(K \in P_{o}(L)\right) .^{8}$ Otherwise $[\rho] \stackrel{K}{\rightarrow}\left[I_{K} \rho\right]$ is green.

Proposition 2.10. Let $n<N-1, \rho \in A(N, n)$ and $K \in\left(\begin{array}{c}{[N]} \\ n+1\end{array}\right)$.

(a) If $[\rho] \stackrel{K}{\rightarrow}\left[I_{K} \rho\right]$ is blue, then the elements of $P_{o}(K)$ are blue in $[\rho]$ and green in $\left[I_{K} \rho\right]$, and the elements of $P_{e}(K)$ are blue in $\left[I_{K} \rho\right]$ and green in $[\rho]$.

(b) If $[\rho] \stackrel{K}{\rightarrow}\left[I_{K} \rho\right]$ is red, then the elements of $P_{e}(K)$ are red in $[\rho]$ and green in $\left[I_{K} \rho\right]$, and the elements of $P_{o}(K)$ are red in $\left[I_{K} \rho\right]$ and green in $[\rho]$.

(c) If $[\rho] \stackrel{K}{\rightarrow}\left[I_{K} \rho\right]$ is green, then all elements of $P(K)$ are green in both, $[\rho]$ and $\left[I_{K} \rho\right]$.

Proof. (a) If $[\rho] \stackrel{K}{\rightarrow}\left[I_{K} \rho\right]$ is blue, this means that for all $L \in E(K)$ either (i) $\vec{P}(L)$ has beginning segments w.r.t. $[\rho]$ and $\left[I_{K} \rho\right]$, and $K \in P_{o}(L)$, or (ii) $\overleftarrow{P}(L)$ has beginning segments w.r.t. [ $\left.\rho\right]$ and $\left[I_{K} \rho\right]$, and $K \in P_{e}(L)$. In case (i), let $J \in P(K)$ and $K^{\prime} \in P(L) \cap E(J)$. If $K^{\prime} \notin \operatorname{inv}\left[I_{K} \rho\right]$, then $K<_{\text {lex }} K^{\prime}$ and thus $J \in P_{o}\left(K^{\prime}\right)$ by Lemma 2.9. If $K^{\prime} \in \operatorname{inv}\left[I_{K} \rho\right]$, then $K^{\prime}<_{\text {lex }} K$, hence $J \in P_{e}\left(K^{\prime}\right)$ according to Lemma 2.9. In both cases we can conclude that, if $J \in P_{o}(K)$, then $J$ is blue in $[\rho]$ and green in $\left[I_{K} \rho\right]$, and if $J \in P_{e}(K)$, then $J$ is blue in $\left[I_{K} \rho\right]$ and green in $[\rho]$. The case (ii) is treated correspondingly.

(b) is proved in the same way.

(c) $[\rho] \stackrel{K}{\rightarrow}\left[I_{K} \rho\right]$ green means that there are $L_{1}, L_{2} \in E(K), L_{1} \neq L_{2}$, such that one of the following three cases holds:

(i) $\vec{P}\left(L_{1}\right)$ and $\vec{P}\left(L_{2}\right)$ have beginning segments w.r.t. $[\rho]$ and $\left[I_{K} \rho\right]$, and $K \in P_{o}\left(L_{1}\right) \cap P_{e}\left(L_{2}\right)$,

(ii) $\vec{P}\left(L_{1}\right)$ and $\overleftarrow{P}\left(L_{2}\right)$ have beginning segments w.r.t. $[\rho]$ and $\left[I_{K} \rho\right]$, and $K \in P_{o}\left(L_{1}\right) \cap P_{o}\left(L_{2}\right)$ or $K \in P_{e}\left(L_{1}\right) \cap P_{e}\left(L_{2}\right)$,

(iii) $\overleftarrow{P}\left(L_{1}\right)$ and $\overleftarrow{P}\left(L_{2}\right)$ have beginning segments w.r.t. $[\rho]$ and $\left[I_{K} \rho\right]$, and $K \in P_{e}\left(L_{1}\right) \cap P_{o}\left(L_{2}\right)$

In case (i), let $J \in P(K)$ and $K_{1} \in P\left(L_{1}\right) \cap E(J), K_{2} \in P\left(L_{2}\right) \cap E(J), K_{1} \neq K_{2}$. If $K<_{\text {lex }} K_{1}, K_{2}$, then $K_{1}, K_{2} \notin \operatorname{inv}\left[I_{K} \rho\right]$, hence $J \in P_{o}\left(K_{1}\right) \cap P_{e}\left(K_{2}\right)$ by Lemma 2.9. If $K_{1}<_{\operatorname{lex}}$ $K<_{\text {lex }} K_{2}$, then $K_{1} \in \operatorname{inv}\left[I_{K} \rho\right]$ and $K_{2} \notin \operatorname{inv}\left[I_{K} \rho\right]$, so that $J \in P_{e}\left(K_{1}\right) \cap P_{e}\left(K_{2}\right)$ by Lemma 2.9 . If $K_{2}<_{\operatorname{lex}} K<_{\operatorname{lex}} K_{1}$, then $K_{1} \notin \operatorname{inv}\left[I_{K} \rho\right]$ and $K_{2} \in \operatorname{inv}\left[I_{K} \rho\right]$, hence $J \in P_{o}\left(K_{1}\right) \cap P_{o}\left(K_{2}\right)$. Finally, if $K_{1}, K_{2}<_{\text {lex }} K$, then $K_{1}, K_{2} \in \operatorname{inv}\left[I_{K} \rho\right]$, hence $J \in P_{e}\left(K_{1}\right) \cap P_{o}\left(K_{2}\right)$ by Lemma 2.9 . In all these cases $J$ is green in both, $[\rho]$ and $\left[I_{K} \rho\right]$. The cases (ii) and (iii) can be treated in a similar way.

The preceding proposition in particular shows that blue (red) elements of $[\rho]$ are not affected by red (blue) and green inversions.

\footnotetext{
${ }^{8}$ Both conditions covered by "has beginning segments with respect to $[\rho]$ and $\left[I_{K} \rho\right]$ " are necessary in order to avoid ambiguities that would otherwise arise if $P(L) \cap \operatorname{inv}[\rho]$ is empty or if $P(L) \cap \operatorname{inv}\left[I_{K} \rho\right]$ is the full packet. We are grateful to one of the referees for pointing this out.
} 
Proposition 2.11. Let $[\rho] \stackrel{K}{\rightarrow}\left[I_{K} \rho\right]$ be blue $(r e d)$ in $B(N, n), n<N$. Then any $J \in P(K)$ that is blue (red) in $[\rho]$ is not blue (red) in any subsequent element of $B(N, n)$.

Proof. This is obvious if $n=N-1$. Let $n<N-1,[\rho] \stackrel{K}{\rightarrow}\left[I_{K} \rho\right]$ and $J \in P(K)$ blue. According to Proposition 2.10, $J$ is green in $\left[\rho_{1}\right]=\left[I_{K} \rho\right]$. Let us assume that $J$ becomes blue again in some subsequent $\left[\rho_{r}\right]$. Then $\left[\rho_{r-1}\right] \stackrel{K_{r}}{\rightarrow}\left[\rho_{r}\right]$ has to be blue and $J \in P_{e}\left(K_{r}\right)$. As a consequence, $K, K_{r} \in E(J), K \neq K_{r}$, and $L=K \cup K_{r}$ has cardinality $n+2$. If $\vec{P}(L)$ has beginning segment w.r.t. $\left[\rho_{r-1}\right]$, then also w.r.t. $\left[\rho_{1}\right]$. Since $K \in P_{o}(L)$ (because $[\rho] \stackrel{K}{\rightarrow}\left[\rho_{1}\right]$ is blue) and $K<_{\text {lex }} K_{r}$, Lemma 2.9 yields $J \in P_{o}\left(K_{r}\right)$ and thus a contradiction. If $\overleftarrow{P}(L)$ has beginning segment w.r.t. $\left[\rho_{r-1}\right]$, then $K \in P_{e}(L)$ and $K_{r}<_{\operatorname{lex}} K$, hence $J \in P_{o}\left(K_{r}\right)$ according to Lemma 2.9, so we have a contradiction. The red case is treated in the same way.

Proposition 2.12. Let $[\alpha]$ and $[\omega]$ be the minimal and the maximal element of $B(N, n)$, respectively. Then the blue (red) elements of $[\alpha]$ are the red (blue) elements of $[\omega]$. Furthermore, $[\alpha]$ and $[\omega]$ share the same green elements.

Proof. Let $J$ be blue in $[\alpha]$. Since $\operatorname{inv}[\alpha]=\varnothing, J$ is blue in $\vec{P}(K)$ for all $K \in E(J)$. Since $\operatorname{inv}[\omega]$ contains all $K, J$ is red in $[\omega]$. Correspondingly, a red $J$ in $[\alpha]$ is blue in $[\omega]$. The last statement of the proposition is then obvious.

Let us recall that we also use $\rho^{(c)}$ to denote the subsequence of $\rho \in A(N, n)$ of color $c$. Now any $\rho \in A(N, n)$ can be decomposed into three subsequences, $\rho^{(b)}, \rho^{(r)}$ and $\rho^{(g)}$, and this decomposition is carried over to $B(N, n)$.

For $K \in\left(\begin{array}{c}{[N]} \\ n+1\end{array}\right)$, let us introduce the half-packet inversions

$$
I_{K}^{(b)}: \vec{P}_{o}(K) \rightarrow \overleftarrow{P_{e}}(K), \quad I_{K}^{(r)}: \vec{P}_{e}(K) \rightarrow \overleftarrow{P_{o}}(K)
$$

Let $\rho \stackrel{K}{\rightarrow} I_{K} \rho$ be an inversion in $A(N, n)$. From the above propositions we conclude:

- if the inversion is blue, then $\left(I_{K} \rho\right)^{(b)}=I_{K}^{(b)} \rho^{(b)},\left(I_{K} \rho\right)^{(r)}=\rho^{(r)}$, and $\left(I_{K} \rho\right)^{(g)}=I_{K}^{(r)} \rho^{(g)}$,

- if the inversion is red, then $\left(I_{K} \rho\right)^{(b)}=\rho^{(b)},\left(I_{K} \rho\right)^{(r)}=I_{K}^{(r)} \rho^{(r)}$, and $\left(I_{K} \rho\right)^{(g)}=I_{K}^{(b)} \rho^{(g)}$,

- if the inversion is green, then $\left(I_{K} \rho\right)^{(b)}=\rho^{(b)},\left(I_{K} \rho\right)^{(r)}=\rho^{(r)}$, and $\left(I_{K} \rho\right)^{(g)}=I_{K} \rho^{(g)}$.

In the following, $B^{(c)}(N, n)$ shall denote the corresponding set supplied with the induced partial order. $B^{(b)}(N, n)$ is the (higher) Tamari order $T(N, n)$ (see [25] for an equivalent definition). $B^{(r)}(N, n)$ is the dual of the (higher) Tamari order $T(N, n) . B^{(g)}(N, n)$ will be called mixed order. The latter involves all the three inversions. It should be noted that a red (blue) halfpacket inversion in $B^{(g)}(N, n)$ stems from a blue (red) inversion $I_{K}$.

Remark 2.13. $B^{(b)}(N, N-1)$ is $\vec{P}_{o}([N]) \stackrel{[N]}{\rightarrow} \overleftarrow{P}_{e}([N])$ and $B^{(r)}(N, N-1)$ is $\vec{P}_{e}([N]) \stackrel{[N]}{\rightarrow} \overleftarrow{P}_{o}([N])$ $B^{(g)}(N, N-1)$ is empty. $B(N, N-2)$ consists of a pair of maximal chains, see (2.1). We set $m:=N \bmod 2$. The two blue subchains

$$
\begin{aligned}
& {\left[\alpha^{(b)}\right] \stackrel{\hat{N}}{\longrightarrow}\left[\rho_{1}^{(b)}\right] \stackrel{\widehat{N-2}}{\longrightarrow}\left[\rho_{3}^{(b)}\right] \longrightarrow \cdots \longrightarrow\left[\rho_{N+m-5}^{(b)}\right] \stackrel{\widehat{4-m}}{\longrightarrow}\left[\rho_{N+m-3}^{(b)}\right] \stackrel{\widehat{2-m}}{\longrightarrow}\left[\omega^{(b)}\right],} \\
& {\left[\alpha^{(b)}\right] \stackrel{\widehat{m+1}}{\longrightarrow}\left[\sigma_{m+1}^{(b)}\right] \stackrel{\widehat{m+3}}{\longrightarrow}\left[\sigma_{m+3}^{(b)}\right] \longrightarrow \cdots \longrightarrow\left[\sigma_{N-5}^{(b)}\right] \stackrel{\widehat{N-3}}{\longrightarrow}\left[\sigma_{N-3}^{(b)}\right] \stackrel{\widehat{N-1}}{\longrightarrow}\left[\omega^{(b)}\right],}
\end{aligned}
$$

constitute $B^{(b)}(N, N-2)$. All inversions are blue, of course. Correspondingly, the two red chains

$$
\left[\alpha^{(r)}\right] \stackrel{\widehat{N-1}}{\longrightarrow}\left[\rho_{2}^{(r)}\right] \stackrel{\widehat{N-3}}{\longrightarrow}\left[\rho_{4}^{(r)}\right] \longrightarrow \cdots \longrightarrow\left[\rho_{N-m-4}^{(r)}\right] \stackrel{\widehat{m+3}}{\longrightarrow}\left[\rho_{N-m-2}^{(r)}\right] \stackrel{\widehat{m+1}}{\longrightarrow}\left[\omega^{(r)}\right],
$$



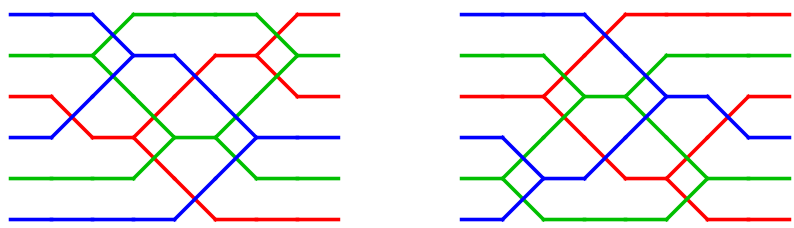

Figure 5. Structure of the chains (2.2). A rhombus with two green and two blue (red) edges corresponds to a blue (red) inversion in $B(4,2)$. Such a rhombus corresponds to a hexagon in $B(4,1)$ (see Remark 5.1).

$$
\left[\alpha^{(r)}\right] \stackrel{\widehat{2-m}}{\longrightarrow}\left[\sigma_{2-m}^{(r)}\right] \stackrel{\widehat{4-m}}{\longrightarrow}\left[\sigma_{4-m}^{(r)}\right] \longrightarrow \cdots \longrightarrow\left[\sigma_{N-4}^{(r)}\right] \stackrel{\widehat{N-2}}{\longrightarrow}\left[\sigma_{N-2}^{(r)}\right] \stackrel{\widehat{N}}{\longrightarrow}\left[\omega^{(r)}\right]
$$

form $B^{(r)}(N, N-2)$. All inversions are red. One maximal chain of $B^{(g)}(N, N-2)$ is

$$
\left[\alpha^{(g)}\right] \stackrel{\hat{N}}{\longrightarrow}\left[\rho_{1}^{(g)}\right] \stackrel{\widehat{N-1}}{\longrightarrow}\left[\rho_{2}^{(g)}\right] \longrightarrow \cdots \longrightarrow\left[\rho_{N-2}^{(g)}\right] \stackrel{\hat{2}}{\longrightarrow}\left[\rho_{N-1}^{(g)}\right] \stackrel{\hat{1}}{\longrightarrow}\left[\omega^{(g)}\right]
$$

where now an inversion $\stackrel{\hat{k}}{\rightarrow}$ is blue if $\hat{k} \in P_{e}([N])$ and red if $\hat{k} \in P_{o}([N])$. The second chain is

$$
\left[\alpha^{(g)}\right] \stackrel{\hat{1}}{\longrightarrow}\left[\sigma_{1}^{(g)}\right] \stackrel{\hat{2}}{\longrightarrow}\left[\sigma_{2}^{(g)}\right] \longrightarrow \cdots \longrightarrow\left[\sigma_{N-2}^{(g)}\right] \stackrel{\widehat{N-1}}{\longrightarrow}\left[\sigma_{N-1}^{(g)}\right] \stackrel{\hat{N}}{\longrightarrow}\left[\omega^{(b)}\right]
$$

where $\stackrel{\hat{k}}{\rightarrow}$ is blue if $\hat{k} \in P_{o}([N])$ and red if $\hat{k} \in P_{e}([N])$. There are no green inversions in this

\begin{tabular}{|c|c|c|c|c|c|c|c|c|c|c|c|}
\hline 12 & 23 & 23 & 23 & 23 & 34 & 12 & 12 & 12 & 34 & 34 & 34 \\
\hline 13 & 13 & 13 & 24 & 24 & 24 & 13 & 13 & 34 & 12 & 24 & 24 \\
\hline $14 \sim 23123$ & 12124 & 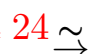 & 13134 & 34234 & 23 & 14234 & 14134 & $14 \sim$ & 14124 & 14123 & $14 \sim$ \\
\hline $\overrightarrow{14} \vec{\imath}$ & $14 \vec{\imath}$ & $14 \vec{\imath}$ & $14 \vec{\rightarrow}$ & $14 \vec{\imath}$ & 14 & 23 & $34 \vec{\longrightarrow}$ & $13 \vec{\imath}$ & $24 \vec{\longrightarrow}$ & $12 \vec{\imath}$ & 23 \\
\hline 24 & 24 & 12 & 34 & 13 & 13 & 24 & 24 & 24 & 13 & 13 & 13 \\
\hline 34 & 34 & 34 & 12 & 12 & 12 & 34 & 23 & 23 & 23 & 23 & 12 \\
\hline
\end{tabular}
case.

Example 2.14. $B(4,2)$ consists of the two maximal chains

Here they are resolved into admissible linear orders, i.e., elements of $A(4,2)$. These are the maximal chains of $B(4,1)$ (forming a permutahedron). The blue and red subchains of $(2.2)$, forming $B^{(b)}(4,2)$ and $B^{(r)}(4,2)$, respectively, are

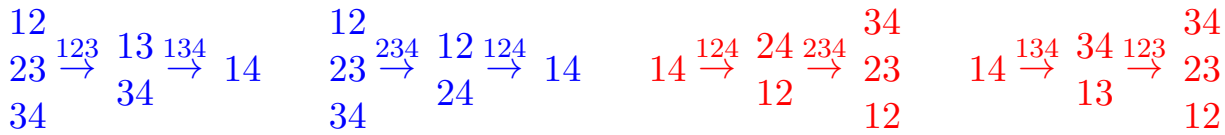

$B^{(g)}(4,2)$ is given by

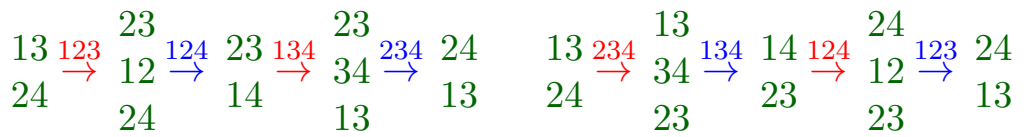

Fig. 5 displays the structure of the two maximal chains $(2.2)$ of $B(4,2) .^{9}$

\footnotetext{
${ }^{9}$ In the context of Soergel bimodules, a corresponding diagrammatic equation (also see Fig. 27) appeared in [31] as the $A_{3}$ Zamolodchikov relation.
} 
Example 2.15. In the following, we display one of the maximal chains of $B(5,2)$, resolved into linear orders (elements of $A(5,2)$ ), and its single-colored subsequences

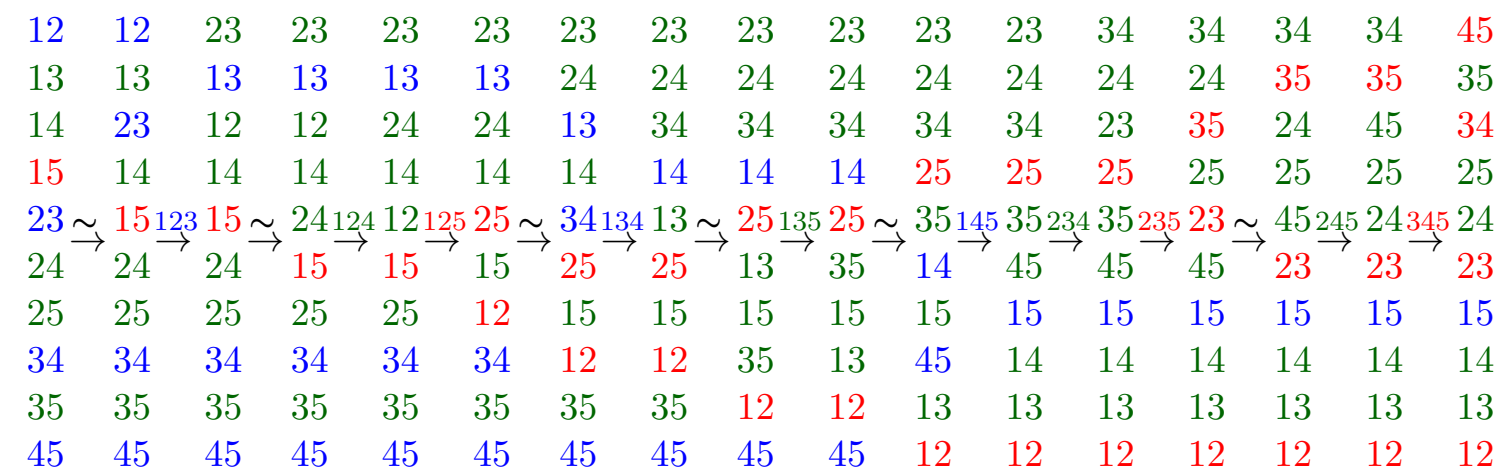

The three subsequences collapse to

\begin{tabular}{|c|c|c|c|c|c|c|c|c|c|c|}
\hline 12 & 13 & & & & & & 45 & & & \\
\hline $23 \stackrel{123}{\longrightarrow}$ & $\begin{array}{l}10 \\
34\end{array}$ & $14 \underset{145}{\rightarrow}$ & 15 & $15 \stackrel{125}{\longrightarrow}$ & $25 \underset{235}{\longrightarrow}$ & $\begin{array}{l}345 \\
23\end{array}$ & 34 & & & \\
\hline $34 \overrightarrow{7}$ & $\begin{array}{l}34 \\
45\end{array}$ & $45 \vec{\longrightarrow}$ & 10 & & $12 \overrightarrow{7}$ & & 23 & & & \\
\hline 45 & & & & & & 23 & $\begin{array}{c}12 \\
34\end{array}$ & & & \\
\hline 13 & 23 & 23 & 23 & 23 & 23 & 24 & 24 & 34 & 34 & 35 \\
\hline & 12 & 24 & 24 & 24 & 24 & & 23 & 24 & 45 & 25 \\
\hline $24 \stackrel{123}{\rightarrow}$ & $14 \stackrel{124}{\rightarrow}$ & $14 \stackrel{125}{\rightarrow}$ & $14 \stackrel{134}{\rightarrow}$ & $34 \stackrel{135}{\rightarrow}$ & $34 \stackrel{145}{\rightarrow}$ & $35 \stackrel{234}{\rightarrow}$ & $35 \stackrel{235}{\rightarrow}$ & $25 \stackrel{245}{\rightarrow}$ & $25 \stackrel{345}{\rightarrow}$ & 24 \\
\hline 25 & 24 & 12 & 15 & 13 & 35 & 45 & 45 & 45 & 24 & 14 \\
\hline & $\begin{array}{l}25 \\
25\end{array}$ & $\begin{array}{l}25 \\
35\end{array}$ & 35 & 15 & 15 & 14 & 14 & 14 & 14 & 13 \\
\hline & & उJ & & & & 13 & 13 & & & \\
\hline
\end{tabular}

Here the blue order is ruled by $I_{i j k}^{(b)}:(i j, j k) \mapsto(i k)$, the red order by $I_{i j k}^{(r)}:(i k) \mapsto(j k, i j)$, where $1 \leq i<j<k \leq 5$. The green order involves these two and in addition $I_{i j k}:(i j, i k, j k) \mapsto$ $(j k, i k, i j)$.

Remark 2.16. Inherited from the Bruhat orders, for $c \in\{b, r\}$, there is a one-to-one correspondence between elements of $A^{(c)}(N, n+1)$ and maximal chains of $B^{(c)}(N, n)$. No such relation exists for the mixed order, but elements of $A^{(g)}(N, n+1)$ are in one-to-one correspondence with the green inversion subsequences of maximal chains of $B^{(g)}(N, n)$.

Remark 2.17. In Remark 2.5 we defined, for each $k \in[N+1]$, a projection $B(N+1, n+1) \rightarrow$ $B(N, n)$, via an equivalence relation $\stackrel{k}{\sim}$. If $k \in[N+1] \backslash\{1, N+1\}$, these projections do not respect the above three color decomposition. The reason is that if $L \in\left(\begin{array}{c}{[N+1]} \\ n+2\end{array}\right)$ contains $k$, then $P_{o}(L) \backslash\{L \backslash\{k\}\}$ and $P_{e}(L) \backslash\{L \backslash\{k\}\}$ cannot be brought into natural correspondence with the half-packets $P_{o}(L \backslash\{k\})$ and $P_{e}(L \backslash\{k\})$, respectively. For example, if $L=1234$ and $k=2$, then $L \backslash\{k\}=134, P_{o}(L)=\{123,134\}, P_{e}(L)=\{124,234\}$, hence $P_{o}(L) \backslash\{L \backslash\{k\}\}=\{123\}$, $P_{e}(L) \backslash\{L \backslash\{k\}\}=\{124,234\}$, while $P_{o}(L \backslash\{k\})=\{13,34\}$ and $P_{e}(L \backslash\{k\})=\{14\}$. But if $k=1$, then $L \backslash\{1\}$ is the last element of $\vec{P}(L)$ and its elimination thus does not influence the positions of the remaining elements. For $k=1$ we therefore obtain monotone projections $B^{(c)}(N+1, n+1) \rightarrow B^{(c)}(N, n)$. The other exception is $k=N+1$. Then $L \backslash\{N+1\}$ is the first element of $\vec{P}(L)$ and its elimination turns odd into even elements, and vice versa. In this case we obtain monotone projections $B^{(b)}(N+1, n+1) \rightarrow B^{(r)}(N, n), B^{(r)}(N+1, n+1) \rightarrow B^{(b)}(N, n)$ and $B^{(g)}(N+1, n+1) \rightarrow B^{(g)}(N, n)$. We will use the projection with $k=1$ in Section 4.4. See, in particular, Figs. 23 and 24. 


\section{Simplex equations}

In this section we consider realizations of Bruhat orders in terms of sets and maps between Carte$\operatorname{sian}^{10}$ products of the sets. The $N$-simplex equation is directly associated with $B(N+1, N-1)$, but its structure is fully displayed as a polyhedral reduction of $B(N+1, N-2)$. Section 3.1 explains the relation with polyhedra and prepares the stage for the definition of simplex equations in Section 3.2, which contains explicit expressions up to the 7-simplex equation, and the associated polyhedra. Section 3.3 discusses the integrability of simplex equations. The reduction of the Bruhat order $B(N+2, N)$ to $B(N+1, N-1)$ induces a reduction of the $(N+1)$-simplex equation to the $N$-simplex equation. This is the subject of Section 3.4.

\subsection{Resolutions of $B(N+1, N-1)$ and polyhedra}

Let $s_{a}$ denote the operation of exchange of elements $J_{a}, J_{a+1}$ of $\rho=\left(J_{1}, \ldots, J_{c(N+1, n)}\right) \in A(N+$ $1, n)$, which is applicable (only) if $E\left(J_{a}\right) \cap E\left(J_{a+1}\right)=\varnothing$. For any $\beta, \beta^{\prime} \in[\rho]$, there is a minimal number $m$ of exchange operations $s_{a_{1}}, \ldots, s_{a_{m}}$, such that $\beta^{\prime}=s_{\beta^{\prime}, \beta} \beta$, where $s_{\beta^{\prime}, \beta}:=s_{a_{m}} \cdots s_{a_{1}}$. The sequence $\beta_{0}, \beta_{1}, \ldots, \beta_{m}$, where $\beta_{0}=\beta, \beta_{i}=s_{a_{i}} \beta_{i-1}, i=1, \ldots, m$, is called a resolution of $[\rho]$ from $\beta$ to $\beta^{\prime}$. It is unique up to potential applications of the identities

$$
s_{a} s_{b}=s_{b} s_{a} \quad \text { if } \quad|a-b|>1, \quad s_{a} s_{a+1} s_{a}=s_{a+1} s_{a} s_{a+1} .
$$

Let $\mathcal{C}:\left[\rho_{0}\right] \stackrel{K_{1}}{\longrightarrow}\left[\rho_{1}\right] \stackrel{K_{2}}{\longrightarrow} \cdots \stackrel{K_{k}}{\longrightarrow}\left[\rho_{k}\right]$ be a chain in $B(N+1, n)$. A resolution $\tilde{\mathcal{C}}$ of $\mathcal{C}$ is a sequence of resolutions of all $\left[\rho_{i}\right]$, such that the initial element of the resolution of $\left[\rho_{i+1}\right]$ is obtained by application of $I_{K_{i+1}}$ to the final element of the resolution of $\left[\rho_{i}\right]$, for $i=0, \ldots, k-1$.

Denoting by $\iota_{a}$ an inversion, acting at positions $a, a+1, \ldots, a+n$, of some element of $A(N+1, n)$, the resolution $\tilde{\mathcal{C}}$ uniquely corresponds to a composition of exchange and inversion operations,

$$
\mathcal{O}_{\tilde{\mathcal{C}}}:=s_{\beta_{k}^{\prime}, \beta_{k}} \iota_{a_{k}} \cdots s_{\beta_{1}^{\prime}, \beta_{1}} \iota_{a_{1}} s_{\beta_{0}^{\prime}, \beta_{0}},
$$

where $\beta_{i}$ is the initial and $\beta_{i}^{\prime}$ the final element of the resolution of $\left[\rho_{i}\right]$, and $\beta_{i+1}=\iota_{a_{i+1}} \beta_{i}^{\prime}$.

Remark 3.1. The operations $s_{a}$ and $\iota_{b}$ satisfy the following identities,

$$
\begin{aligned}
& s_{a} \iota_{b}=\iota_{b} s_{a} \quad \text { if } \quad a<b-1 \text { or } a>b+n, \\
& \iota_{a} \iota_{b}=\iota_{b} \iota_{a} \quad \text { if } \quad|b-a|>n, \\
& \iota_{a} s_{a+n} \cdots s_{a+1} s_{a}=s_{a+n} \cdots s_{a+1} s_{a} \iota_{a+1} .
\end{aligned}
$$

In the last identity, $s_{a+n} \cdots s_{a+1} s_{a}$ exchanges the element at position $a$ with the block of elements at positions $a+1, \ldots, a+n+1$. The identities (3.3) take care of the fact that the above definition of a resolution of a chain in $B(N+1, n)$ does not in general fix all the final elements of the resolutions of the $\left[\rho_{i}\right]$. Using $s_{a}^{2}=\mathrm{id}$, in the last of the above relations one can move exchange operations from one side to the other. Since the relations (3.1) and (3.3) are homogeneous, all resolutions with the same initial and the same final element have the same length.

The Bruhat order $B(N+1, N-1)$ consists of the two maximal chains ${ }^{11}$

$$
\mathcal{C}_{\text {lex }}:[\alpha] \stackrel{[N+1] \backslash\{N+1\}}{\longrightarrow}\left[\rho_{1}\right] \stackrel{[N+1] \backslash\{N\}}{\longrightarrow} \cdots \stackrel{[N+1] \backslash\{2\}}{\longrightarrow}\left[\rho_{N}\right] \stackrel{[N+1] \backslash\{1\}}{\longrightarrow}[\omega],
$$

\footnotetext{
${ }^{10}$ Alternatively, we may as well consider tensor products or direct sums, assuming that the sets carry the necessary additional structure.

${ }^{11}$ Here "lex" and "rev" stand for "lexicographically ordered" and "reverse lexicographically ordered", respectively. In these chains we should better use complementary notation, $\hat{l}:=[N+1] \backslash\{l\}$, and we will do this mostly in the following.
} 

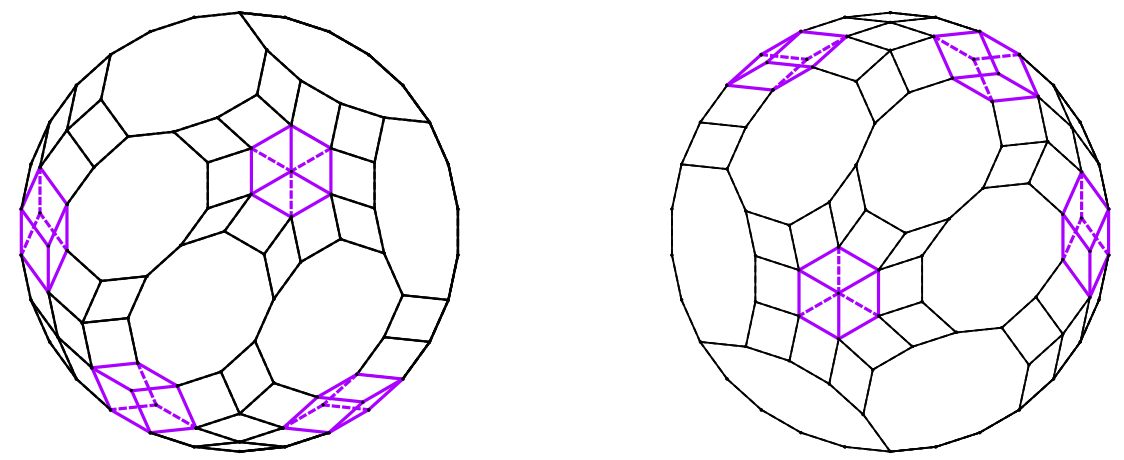

Figure 6. Complementary sides of $B(6,3)$. Because of the "small cubes" (here marked purple), $B(6,3)$ is not polyhedral.

$$
\mathcal{C}_{\mathrm{rev}}:[\alpha] \stackrel{[N+1] \backslash\{1\}}{\longrightarrow}\left[\sigma_{1}\right] \stackrel{[N+1] \backslash\{2\}}{\longrightarrow} \cdots \stackrel{[N+1] \backslash\{N\}}{\longrightarrow}\left[\sigma_{N}\right] \stackrel{[N+1] \backslash\{N+1\}}{\longrightarrow}[\omega]
$$

This implies that there are resolutions $\tilde{\mathcal{C}}_{\text {lex }}$ and $\tilde{\mathcal{C}}_{\text {rev }}$ of $\mathcal{C}_{\text {lex }}$ and $\mathcal{C}_{\text {rev }}$, respectively, both starting with $\alpha$ and both ending with $\omega$,

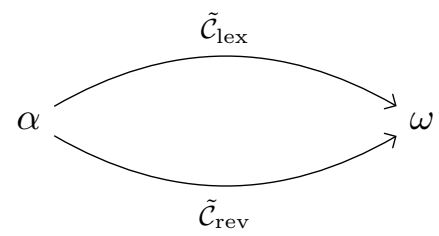

Via the correspondence between elements of $A(N+1, N-1)$ and maximal chains of $B(N+$ $1, N-2$ ) (see Remark 2.2), each of the two resolutions corresponds to a sequence of maximal chains of $B(N+1, N-2)$. For $\rho \in A(N+1, N-1)$, let $\mathcal{C}_{\rho}$ be the corresponding maximal chain of $B(N+1, N-2)$. $\tilde{\mathcal{C}}_{\text {lex }}$, respectively $\tilde{\mathcal{C}}_{\text {rev }}$, is then a rule for deforming $\mathcal{C}_{\alpha}$ stepwise into $\mathcal{C}_{\omega}$.

Moreover, a resolution of $B(N+1, N-1)$, represented by the above diagram, contains a rule to construct a polyhedron. Starting from a common vertex, we represent the elements of $\alpha$ and $\omega=\operatorname{rev}(\alpha)$ ( $\alpha$ in reversed order $)$ from top to bottom as the edges of the left, respectively right side of a regular $N(N+1)$-gon. Then we deform the left side (corresponding to $\alpha$ ) stepwise, following the resolution $\tilde{\mathcal{C}}_{\text {lex }}$ and ending in the right side (corresponding to $\omega$ ) of the polygon. For any appearance of an exchange operation $s$ we insert a rhombus, and for any inversion $\iota$ a $2 N$-gon. This is done in such a way that opposite edges are parallel and have equal length, so the inserted polygons are zonogons. We proceed in the same way with the resolution $\tilde{\mathcal{C}}_{\text {rev }}$. The resulting two zonotiles constitute complementary sides of a zonohedron. Up to "small cubes" (see the following remark), it represents $B(N+1, N-2)$.

Remark 3.2. For the first few values of $N, B(N+1, N-2)$ forms a polyhedron. This is no longer so for higher values, because "small cubes" appear [38]. Fig. 6 displays them for $B(6,3)$. A small cube is present in the Bruhat order $B(N+1, N-2)$ whenever there are two different resolutions of an element of $B(N+1, N-1)$, which are identical except that one of them contains a subsequence $\beta, s_{a} \beta, s_{a+1} s_{a} \beta, \beta^{\prime}$ and the other $\beta, s_{a+1} \beta, s_{a} s_{a+1} \beta, \beta^{\prime}$ instead, where $\beta^{\prime}=s_{a} s_{a+1} s_{a} \beta \equiv s_{a+1} s_{a} s_{a+1} \beta$. The six members of the two subsequences determine six maximal chains of $B(N+1, N-2)$, which enclose a cube (similarly as in Fig. 1). The process of deformations of maximal chains described above keeps only half of any small cube. The polyhedron, constructed in the way described above, is a polyhedral reduction of $B(N+1, N-2)$. 


\subsection{Simplex equations and associated polyhedra}

With each $J \in\left(\begin{array}{c}{[N+1]} \\ n\end{array}\right)$, we associate a set $\mathcal{U}_{J}$. With $\rho \in A(N+1, n), \rho=\left(J_{1}, \ldots, J_{c(N+1, n)}\right)$, we then associate the Cartesian product

$$
\mathcal{U}_{\rho}:=\mathcal{U}_{J_{1}} \times \mathcal{U}_{J_{2}} \times \cdots \times \mathcal{U}_{J_{c(N+1, n)}}
$$

Furthermore, for each $K \in\left(\begin{array}{c}{[N+1]} \\ n+1\end{array}\right)$, let there be a map

$$
\mathcal{R}_{K}: \mathcal{U}_{\vec{P}(K)} \rightarrow \mathcal{U}_{\overleftarrow{P}(K)}
$$

If $\mathcal{U}_{\vec{P}(K)}$ appears in $\mathcal{U}_{\rho}$ at consecutive positions, starting at position $\boldsymbol{a}$, we extend $\mathcal{R}_{K}$ to a map $\mathcal{R}_{K, a}: \mathcal{U}_{\rho} \rightarrow \mathcal{U}_{\rho^{\prime}}$, where it acts non-trivially only on the sets labeled by the elements of $P(K)$. $\mathcal{R}_{K, \boldsymbol{a}}$ then represents the inversion operation $\iota_{a}$. The exchange operation $s_{a}$ will be represented by the transposition map $\mathcal{P}_{\boldsymbol{a}}$ (where $\mathcal{P}:(u, v) \mapsto(v, u)$ ), which acts at positions $\boldsymbol{a}$ and $\boldsymbol{a}+1$ of $\mathcal{U}_{\rho} \cdot{ }^{12}$ In this way, the resolution $\tilde{\mathcal{C}}$ of the chain $\mathcal{C}$ considered in Section 3.1 translates, via (3.2), to a composition of maps,

$$
\mathcal{R}_{\tilde{\mathcal{C}}}:=\mathcal{P}_{\beta_{k}^{\prime}, \beta_{k}} \mathcal{R}_{K_{k}, a_{k}} \cdots \mathcal{P}_{\beta_{1}^{\prime}, \beta_{1}} \mathcal{R}_{K_{1}, a_{1}} \mathcal{P}_{\beta_{0}^{\prime}, \beta_{0}}
$$

where $\mathcal{P}_{\beta_{i}^{\prime}, \beta_{i}}=\mathcal{P}_{\boldsymbol{a}_{i, m_{i}}} \cdots \mathcal{P}_{\boldsymbol{a}_{i, 1}}$.

Let us now turn to $B(N+1, N-1)$. Choosing $\alpha$ as the lexicographically ordered set $\left(\begin{array}{c}{[N+1]} \\ N-1\end{array}\right)$, and $\omega$ as $\alpha$ in reverse order, we define the $N$-simplex equation as

$$
\mathcal{R}_{\tilde{\mathcal{C}}_{\text {lex }}}=\mathcal{R}_{\tilde{\mathcal{C}}_{\text {rev }}},
$$

where $\tilde{\mathcal{C}}_{\text {lex }}$ and $\tilde{\mathcal{C}}_{\text {rev }}$ are resolutions of $\mathcal{C}_{\text {lex }}$ and $\mathcal{C}_{\text {rev }}$, respectively, with initial element $\alpha$ and final element $\omega$, and $\mathcal{R}_{\tilde{\mathcal{C}}_{\text {lex }}}, \mathcal{R}_{\tilde{\mathcal{C}}_{\text {rev }}}$ are the corresponding compositions of maps $\mathcal{R}_{K, \boldsymbol{a}}, \mathcal{P}_{\boldsymbol{b}}$. (3.4) is independent of the choices of the resolutions $\tilde{\mathcal{C}}_{\text {lex }}$ and $\tilde{\mathcal{C}}_{\text {rev }}$, since $\mathcal{P}_{\boldsymbol{a}}$ and $\mathcal{R}_{K, \boldsymbol{b}}$ clearly satisfy all the relations that $s_{a}$ and $\iota_{b}$ fulfill (see Section 3.1). Since we have the freedom to move a transposition in leftmost or rightmost position from one side of (3.4) to the other, the above choice of $\alpha$ is no restriction.

Remark 3.3. Let

$$
\mathcal{P}_{K}: \mathcal{U}_{\overleftarrow{P}(K)} \rightarrow \mathcal{U}_{\vec{P}(K)}
$$

be a composition of transposition maps $\mathcal{P}_{\boldsymbol{a}}$ corresponding to a reversion. The maps $\hat{\mathcal{R}}_{K}$ that we will encounter in this section are related to the respective maps $\mathcal{R}_{K}$ via

$$
\hat{\mathcal{R}}_{K}=\mathcal{R}_{K} \mathcal{P}_{K}
$$

and they are endomorphisms

$$
\hat{\mathcal{R}}_{K}: \mathcal{U}_{\overleftarrow{P}(K)} \longrightarrow \mathcal{U}_{\overleftarrow{P}(K)}
$$

$\mathcal{R}_{K}$ acts on $\mathcal{U}_{\rho}$ only if $\vec{P}(K)$ appears at consecutive positions in $\rho$, and it changes the order of the factors of $\mathcal{U}_{\rho}$. In contrast, $\hat{\mathcal{R}}_{K}$ only acts on $\mathcal{U}_{\omega}$ (not necessarily at consecutive positions). It does not change the order of $\mathcal{U}$ 's.

\footnotetext{
${ }^{12}$ We use boldface "position" numbers in order to distinguish them more clearly from the numbers specifying some $K \in\left(\begin{array}{c}{[N+1]} \\ n+1\end{array}\right)$.
} 
In complementary notation, the reverse lexicographical order $\omega$ on $\left(\begin{array}{c}{[N+1]} \\ N-1\end{array}\right)$ reads

$$
\omega=(\widehat{12}, \widehat{13}, \ldots, 1 \widehat{(N+1)}, \widehat{23}, \widehat{24}, \ldots, 2 \widehat{2(\widehat{N+1})}, \ldots, N \widehat{(N+1)}) .
$$

The $N$-simplex equation has the form

$$
\hat{\mathcal{R}}_{\hat{1}, \boldsymbol{A}_{1}} \hat{\mathcal{R}}_{\hat{2}, \boldsymbol{A}_{2}} \cdots \hat{\mathcal{R}}_{\widehat{N+1}, \boldsymbol{A}_{N+1}}=\hat{\mathcal{R}}_{\widehat{N+1}, \boldsymbol{A}_{N+1}} \hat{\mathcal{R}}_{\hat{N}, \boldsymbol{A}_{N}} \cdots \hat{\mathcal{R}}_{\hat{1}, \boldsymbol{A}_{1}},
$$

where both sides are maps $\mathcal{U}_{\omega} \rightarrow \mathcal{U}_{\omega}$. One has to determine the positions, given by the multiindex $\boldsymbol{A}_{k}$, of the factors of $\mathcal{U}_{\omega}$, on which the map $\hat{\mathcal{R}}_{\hat{k}}$ acts. For the examples in this section, it is given by $\boldsymbol{A}_{k}=\left(\boldsymbol{a}_{k, 1}, \ldots, \boldsymbol{a}_{k, N+1}\right)$, where the integers $\boldsymbol{a}_{k, j}$ are determined by

$$
\boldsymbol{a}_{k, j}= \begin{cases}\frac{1}{2}(2 n-k)(k-1)+j & \text { if } k \leq j, \\ \boldsymbol{a}_{j, k-1} & \text { if } k>j .\end{cases}
$$

1-simplex equation. In case of $B(2,0)$ we consider maps $\mathcal{R}_{1}, \mathcal{R}_{2}: \mathcal{U}_{\varnothing} \longrightarrow \mathcal{U}_{\varnothing}$ subject to

$$
\mathcal{R}_{2} \mathcal{R}_{1}=\mathcal{R}_{1} \mathcal{R}_{2}
$$

which is the 1-simplex equation.

2-simplex equation and the cube. Associated with the two maximal chains of $B(3,1)$ is the 2-simplex, or Yang-Baxter equation,

$$
\mathcal{R}_{23, \mathbf{1}} \mathcal{R}_{13, \mathbf{2}} \mathcal{R}_{12, \mathbf{1}}=\mathcal{R}_{12, \mathbf{2}} \mathcal{R}_{13, \mathbf{1}} \mathcal{R}_{23, \mathbf{2}},
$$

for maps $\mathcal{R}_{i j}: \mathcal{U}_{i} \times \mathcal{U}_{j} \rightarrow \mathcal{U}_{j} \times \mathcal{U}_{i}, i<j$. The two sides of this equation correspond to sequences of maximal chains on two complementary sides of the cube, formed by $B(3,0)$, see Fig. 1 . In complementary notation, $23=\hat{1}, 13=\hat{2}$ and $12=\hat{3}$, the Yang-Baxter equation reads

$$
\mathcal{R}_{\hat{1}, 1} \mathcal{R}_{\hat{2}, 2} \mathcal{R}_{\hat{3}, 1}=\mathcal{R}_{\hat{3}, 2} \mathcal{R}_{\hat{2}, 1} \mathcal{R}_{\hat{1}, 2} .
$$

In terms of $\hat{\mathcal{R}}_{\hat{k}}:=\mathcal{R}_{\hat{k}} \mathcal{P}$, it takes the form

$$
\hat{\mathcal{R}}_{\hat{1}, \mathbf{1 2}} \hat{\mathcal{R}}_{\hat{2}, \mathbf{1 3}} \hat{\mathcal{R}}_{\hat{3}, \mathbf{2 3}}=\hat{\mathcal{R}}_{\hat{3}, \mathbf{2 3}} \hat{\mathcal{R}}_{\hat{2}, \mathbf{1 3}} \hat{\mathcal{R}}_{\hat{1}, \mathbf{1 2}} \text {. }
$$

3-simplex equation and the permutahedron. The two maximal chains of $B(4,2)$ are

$$
\mathcal{C}_{\text {lex }}:[\alpha] \stackrel{123}{\rightarrow}\left[\rho_{1}\right] \stackrel{124}{\rightarrow}\left[\rho_{2}\right] \stackrel{134}{\rightarrow}\left[\rho_{3}\right] \stackrel{234}{\rightarrow}[\omega], \quad \mathcal{C}_{\mathrm{rev}}:[\alpha] \stackrel{234}{\rightarrow}\left[\sigma_{1}\right] \stackrel{134}{\rightarrow}\left[\sigma_{2}\right] \stackrel{124}{\rightarrow}\left[\sigma_{3}\right] \stackrel{123}{\rightarrow}[\omega] .
$$

Let us start with the lexicographical linear order $\alpha=(12,13,14,23,24,34)$. The minimal element $[\alpha] \in B(4,2)$ also contains $(12,13,23,14,24,34)$. $\omega$ is $\alpha$ in reverse order. We already displayed $\tilde{\mathcal{C}}_{\text {lex }}$ and $\tilde{\mathcal{C}}_{\text {rev }}$ in $(2.2)$. From them we read off

$$
\mathcal{R}_{\tilde{\mathcal{C}}_{\text {lex }}}=\mathcal{R}_{234, \mathbf{1}} \mathcal{R}_{134, \mathbf{3}} \mathcal{P}_{\mathbf{5}} \mathcal{P}_{\mathbf{2}} \mathcal{R}_{124, \mathbf{3}} \mathcal{R}_{123, \mathbf{1}} \mathcal{P}_{\mathbf{3}}, \quad \mathcal{R}_{\tilde{\mathcal{C}}_{\text {rev }}}=\mathcal{P}_{\mathbf{3}} \mathcal{R}_{123,4} \mathcal{R}_{124, \mathbf{2}} \mathcal{P}_{\mathbf{4}} \mathcal{P}_{\mathbf{1}} \mathcal{R}_{134, \mathbf{2}} \mathcal{R}_{234, \mathbf{4}},
$$

for maps $\mathcal{R}_{i j k}: \mathcal{U}_{i j} \times \mathcal{U}_{i k} \times \mathcal{U}_{j k} \rightarrow \mathcal{U}_{j k} \times \mathcal{U}_{i k} \times \mathcal{U}_{i j}, i<j<k$. This determines the 3 -simplex equation

$$
\mathcal{R}_{234, \mathbf{1}} \mathcal{R}_{134, \mathbf{3}} \mathcal{P}_{\mathbf{5}} \mathcal{P}_{\mathbf{2}} \mathcal{R}_{124, \mathbf{3}} \mathcal{R}_{123, \mathbf{1}} \mathcal{P}_{\mathbf{3}}=\mathcal{P}_{\mathbf{3}} \mathcal{R}_{123, \mathbf{4}} \mathcal{R}_{124, \mathbf{2}} \mathcal{P}_{\mathbf{4}} \mathcal{P}_{\mathbf{1}} \mathcal{R}_{134, \mathbf{2}} \mathcal{R}_{234, \mathbf{4}}
$$

In complementary notation, $234=\hat{1}, 134=\hat{2}$, etc., it reads

$$
\mathcal{R}_{\hat{1}, \mathbf{1}} \mathcal{R}_{\hat{2}, \mathbf{3}} \mathcal{P}_{\mathbf{5}} \mathcal{P}_{\mathbf{2}} \mathcal{R}_{\hat{3}, \mathbf{3}} \mathcal{R}_{\hat{4}, \mathbf{1}} \mathcal{P}_{\mathbf{3}}=\mathcal{P}_{\mathbf{3}} \mathcal{R}_{\hat{4}, \mathbf{4}} \mathcal{R}_{\hat{3}, \mathbf{2}} \mathcal{P}_{\mathbf{4}} \mathcal{P}_{\mathbf{1}} \mathcal{R}_{\hat{2}, \mathbf{2}} \mathcal{R}_{\hat{1}, \mathbf{4}},
$$




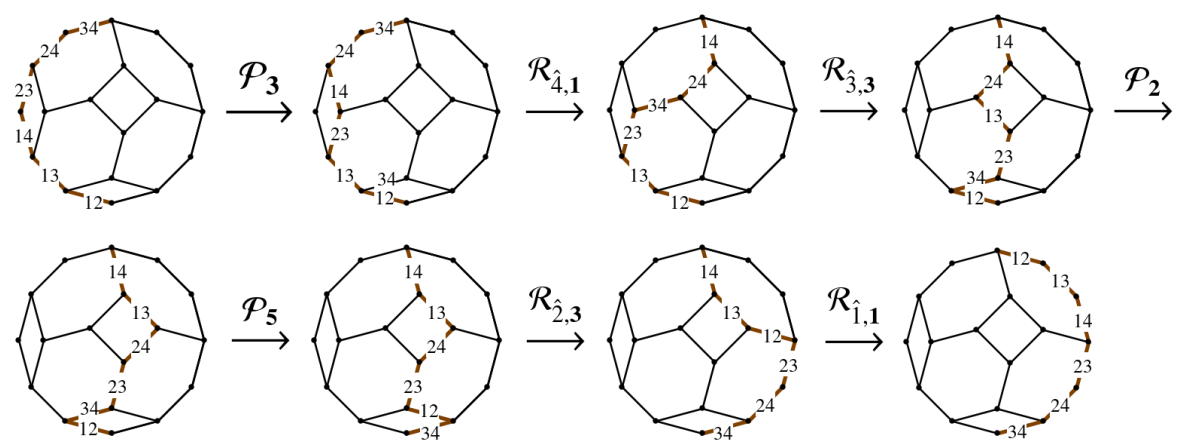

Figure 7. The left-hand side of the 3-simplex equation (3.5) corresponds to a sequence of maximal chains of $B(4,1)$. This sequence forms one side of the permutahedron in three dimensions. Here and in the following figures, if not stated otherwise, edge labels in graphs will be in complementary notation, but with hats omitted.

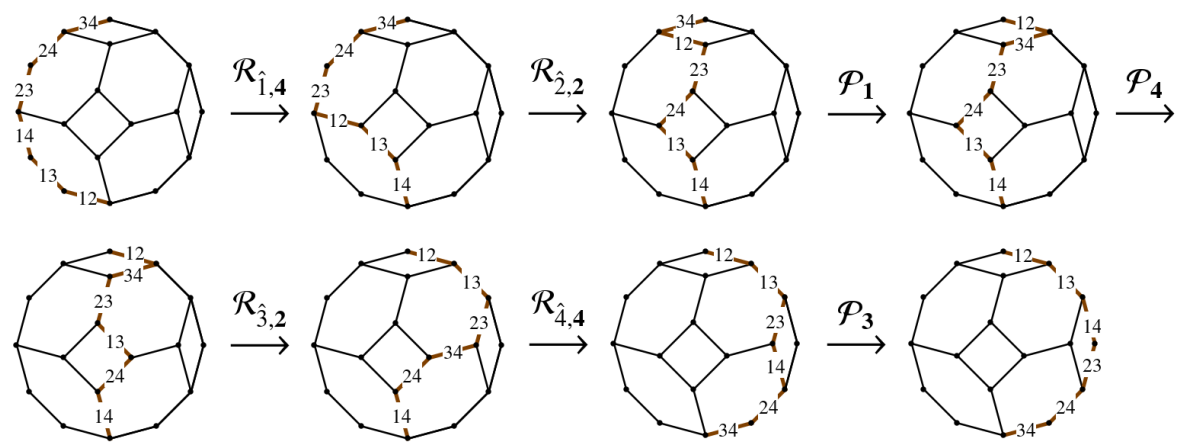

Figure 8. The right-hand side of the 3-simplex equation (3.5) corresponds to a sequence of maximal chains of $B(4,1)$, forming the side of the permutahedron complementary to that in Fig. 7 .

where, for example, $\mathcal{R}_{\hat{1}}: \mathcal{U}_{\widehat{14}} \times \mathcal{U}_{\widehat{13}} \times \mathcal{U}_{\widehat{12}} \rightarrow \mathcal{U}_{\widehat{12}} \times \mathcal{U}_{\widehat{13}} \times \mathcal{U}_{\widehat{14}}$ and $\mathcal{R}_{\hat{2}}: \mathcal{U}_{\widehat{24}} \times \mathcal{U}_{\widehat{23}} \times \mathcal{U}_{\widehat{12}} \rightarrow$ $\mathcal{U}_{\widehat{12}} \times \mathcal{U}_{\widehat{23}} \times \mathcal{U}_{\widehat{24}}$. Left- and right-hand side of (3.5) correspond, respectively, to Figs. 7 and 8 . Collapsing the sequences of graphs in these figures, we can represent the equation as in Fig. 9. Disregarding the indices associated with the underlying Bruhat order, this is Fig. 17 in [70] and Fig. 5 in Chapter 6 of [21], where the 3-simplex equation has been called "permutohedron equation".

In terms of $\hat{\mathcal{R}}:=\mathcal{R} \mathcal{P}_{\mathbf{1 3}}$, where $\mathcal{P}_{\boldsymbol{a} \boldsymbol{b}}$ is the transposition map acting at positions $\boldsymbol{a}$ and $\boldsymbol{b}$, the 3 -simplex equation takes the form

$$
\hat{\mathcal{R}}_{\hat{1}, \mathbf{1 2 3}} \hat{\mathcal{R}}_{\hat{2}, \mathbf{1 4 5}} \hat{\mathcal{R}}_{\hat{3}, \mathbf{2 4 6}} \hat{\mathcal{R}}_{\hat{4}, \mathbf{3 5 6}}=\hat{\mathcal{R}}_{\hat{4}, \mathbf{3 5 6}} \hat{\mathcal{R}}_{\hat{3}, \mathbf{2 4 6}} \hat{\mathcal{R}}_{\hat{2}, \mathbf{1 4 5}} \hat{\mathcal{R}}_{\hat{1}, \mathbf{1 2 3}},
$$

which is also known as the tetrahedron or Zamolodchikov equation. Ignoring the boldface indices and interpreting the others as "position indices", we formally obtain the Frenkel-Moore version [39]

$$
\hat{\mathcal{R}}_{234} \hat{\mathcal{R}}_{134} \hat{\mathcal{R}}_{124} \hat{\mathcal{R}}_{123}=\hat{\mathcal{R}}_{123} \hat{\mathcal{R}}_{124} \hat{\mathcal{R}}_{134} \hat{\mathcal{R}}_{234} .
$$

With a different interpretation of the indices, this equation appeared, for example, in [20].

4-simplex equation and the Felsner-Ziegler polyhedron. In case of $B(5,3)$ we consider maps $\mathcal{R}_{i j k l}: \mathcal{U}_{i j k} \times \mathcal{U}_{i j l} \times \mathcal{U}_{i k l} \times \mathcal{U}_{j k l} \rightarrow \mathcal{U}_{j k l} \times \mathcal{U}_{i k l} \times \mathcal{U}_{i j l} \times \mathcal{U}_{i j k}, i<j<k<l$. Turning to complementary notation, we have, for example, $\mathcal{R}_{2345}=\mathcal{R}_{\hat{1}}: \mathcal{U}_{\widehat{15}} \times \mathcal{U}_{\widehat{14}} \times \mathcal{U}_{\widehat{13}} \times \mathcal{U}_{\widehat{12}} \rightarrow$ 


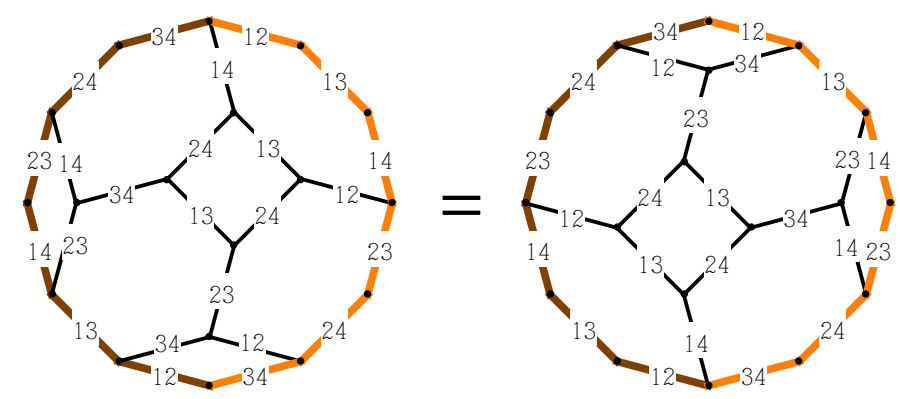

Figure 9. Graphical representation of the 3 -simplex equation.
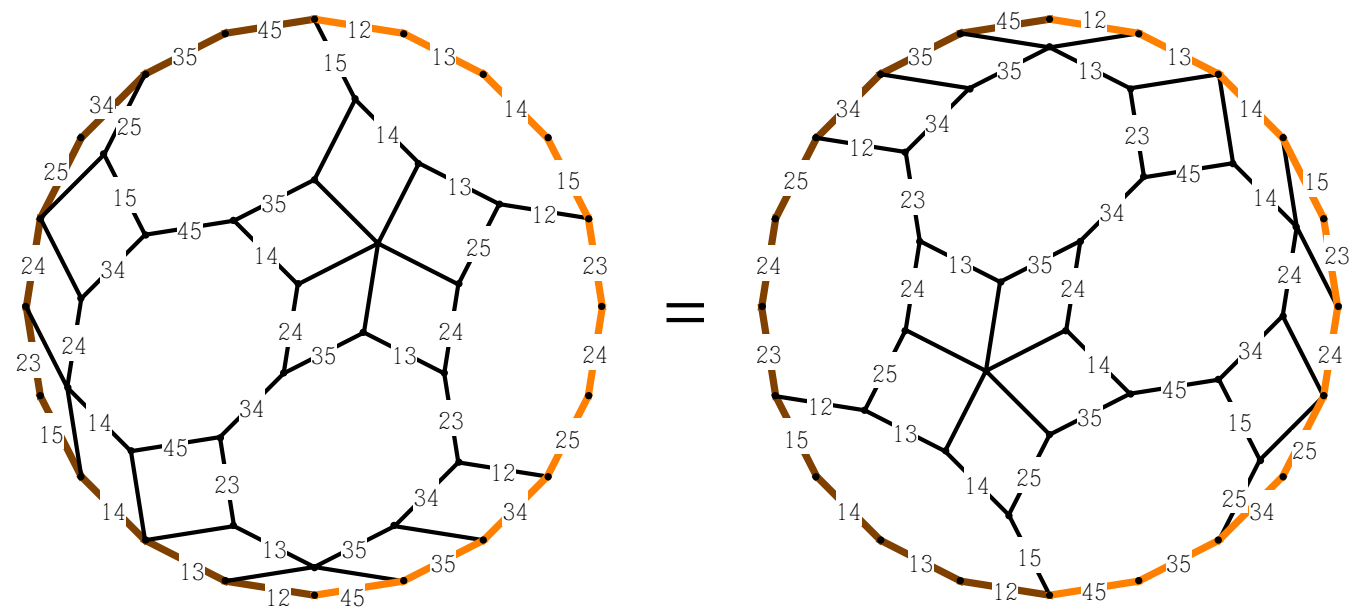

Figure 10. The two sides of the 4-simplex equation correspond to sequences of maximal chains on two complementary sides (left and right figure) of the Felsner-Ziegler polyhedron, which carries the partial order $B(5,2)$. Edge labels are in complementary notation, but with hats omitted.

$\mathcal{U}_{\widehat{12}} \times \mathcal{U}_{\widehat{13}} \times \mathcal{U}_{\widehat{14}} \times \mathcal{U}_{\widehat{15}}$. The maps are subject to the 4 -simplex equation

$$
\begin{aligned}
\mathcal{R}_{\hat{1}, \mathbf{1}} \mathcal{R}_{\hat{2}, 4} \mathcal{P}_{\mathbf{7}} \mathcal{P}_{\mathbf{8}} \mathcal{P}_{\mathbf{9}} \mathcal{P}_{\mathbf{3}} \mathcal{P}_{\mathbf{2}} \mathcal{P}_{\mathbf{4}} \mathcal{R}_{\hat{3}, \mathbf{5}} \mathcal{P}_{\mathbf{8}} \mathcal{P}_{\mathbf{7}} \mathcal{P}_{\mathbf{4}} \mathcal{P}_{\mathbf{3}} \mathcal{R}_{\hat{4}, 4} \mathcal{P}_{\mathbf{7}} \mathcal{R}_{\hat{5}, \mathbf{1}} \mathcal{P}_{\mathbf{4}} \mathcal{P}_{\mathbf{5}} \mathcal{P}_{\mathbf{6}} \mathcal{P}_{\mathbf{3}} \\
=\mathcal{P}_{\mathbf{7}} \mathcal{P}_{\mathbf{4}} \mathcal{P}_{\mathbf{5}} \mathcal{P}_{\mathbf{6}} \mathcal{P}_{\mathbf{3}} \mathcal{R}_{\hat{5}, \mathbf{7}} \mathcal{R}_{\hat{4}, 4} \mathcal{P}_{\mathbf{7}} \mathcal{P}_{\mathbf{8}} \mathcal{P}_{\mathbf{6}} \mathcal{P}_{\mathbf{3}} \mathcal{P}_{\mathbf{2}} \mathcal{P}_{\mathbf{1}} \mathcal{R}_{\hat{3}, \mathbf{3}} \mathcal{P}_{\mathbf{6}} \mathcal{P}_{\mathbf{7}} \mathcal{P}_{\mathbf{2}} \mathcal{P}_{\mathbf{3}} \mathcal{R}_{\hat{2}, 4} \mathcal{R}_{\hat{1}, \mathbf{7}}
\end{aligned}
$$

This can be read off from $B(5,2)$, which forms the Felsner-Ziegler polyhedron $\left(G_{5}\right.$ in [38]), see Fig. 10. In terms of $\hat{\mathcal{R}}_{\hat{k}}:=\mathcal{R}_{\hat{k}} \mathcal{P}_{\mathbf{2 3}} \mathcal{P}_{\mathbf{1 4}}$, the 4 -simplex equation takes the more concise form

$$
\begin{aligned}
& \hat{\mathcal{R}}_{\hat{1}, \mathbf{1}, \mathbf{2}, \mathbf{3}, \mathbf{4}} \hat{\mathcal{R}}_{\hat{2}, \mathbf{1}, \mathbf{5}, \mathbf{6}, \mathbf{7}} \hat{\mathcal{R}}_{\hat{3}, \mathbf{2}, \mathbf{5}, \mathbf{8}, \mathbf{9}} \hat{\mathcal{R}}_{\hat{4}, \mathbf{3}, \mathbf{6}, \mathbf{8}, \mathbf{1 0}} \hat{\mathcal{R}}_{\hat{5}, \mathbf{4}, \mathbf{7}, \mathbf{9}, \mathbf{1 0}}
\end{aligned}
$$

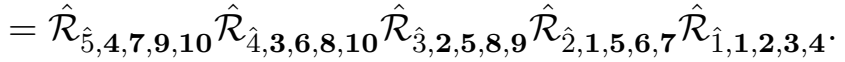

It is also known as the Bazhanov-Stroganov equation (see, e.g., [76]).

5-simplex equation. Turning to $B(6,4)$, we are dealing with maps

$$
\mathcal{R}_{i j k l m}: \mathcal{U}_{i j k l} \times \mathcal{U}_{i j k m} \times \mathcal{U}_{i j l m} \times \mathcal{U}_{i k l m} \times \mathcal{U}_{j k l m} \longrightarrow \mathcal{U}_{j k l m} \times \mathcal{U}_{i k l m} \times \mathcal{U}_{i j l m} \times \mathcal{U}_{i j k m} \times \mathcal{U}_{i j k l},
$$

where $i<j<k<l<m$. In complementary notation, for example,

$$
\mathcal{R}_{23456}=\mathcal{R}_{\hat{1}}: \mathcal{U}_{\widehat{16}} \times \mathcal{U}_{\widehat{15}} \times \mathcal{U}_{\widehat{14}} \times \mathcal{U}_{\widehat{13}} \times \mathcal{U}_{\widehat{12}} \longrightarrow \mathcal{U}_{\widehat{12}} \times \mathcal{U}_{\widehat{13}} \times \mathcal{U}_{\widehat{14}} \times \mathcal{U}_{\widehat{15}} \times \mathcal{U}_{\widehat{16}}
$$




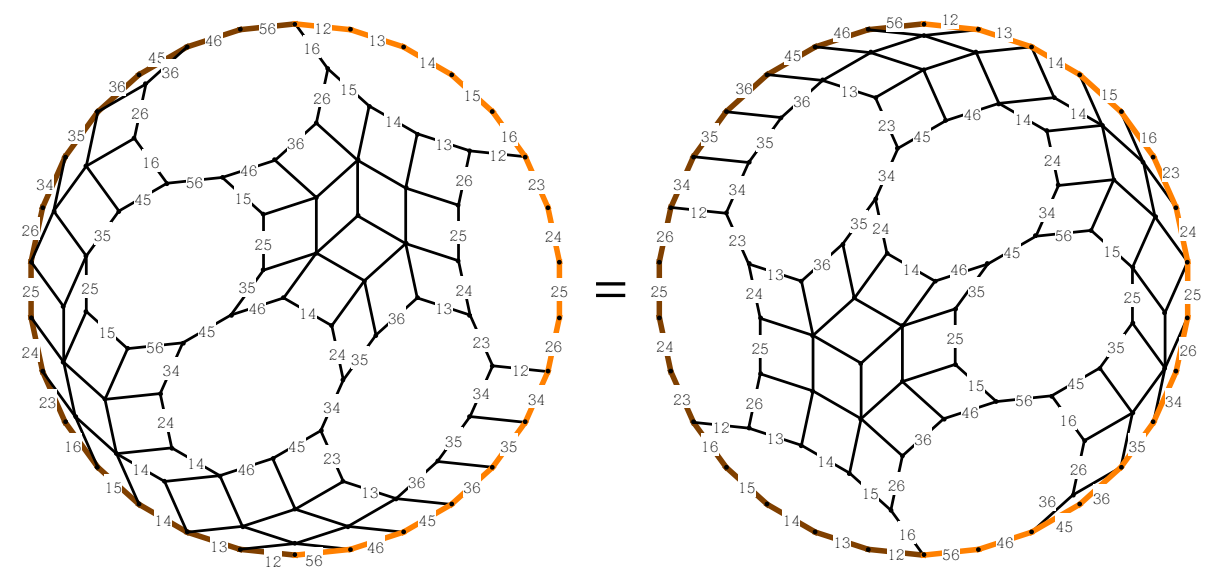

Figure 11. The two sides of the 5 -simplex equation correspond to sequences of maximal chains on two complementary sides (left and right figure) of a polyhedral reduction of $B(6,3)$. Parallel edges carry the same label.

These maps have to satisfy the 5-simplex equation

$$
\begin{aligned}
\mathcal{R}_{\hat{1}, 1} \mathcal{R}_{\hat{2}, 5} \mathcal{P}_{9} \mathcal{P}_{10} \mathcal{P}_{11} \mathcal{P}_{12} \mathcal{P}_{13} \mathcal{P}_{14} \mathcal{P}_{4} \mathcal{P}_{3} \mathcal{P}_{2} \mathcal{P}_{5} \mathcal{P}_{4} \mathcal{P}_{6} \mathcal{R}_{\hat{3}, 7} \mathcal{P}_{11} \mathcal{P}_{12} \mathcal{P}_{13} \mathcal{P}_{10} \\
\mathcal{P}_{11} \mathcal{P}_{12} \mathcal{P}_{6} \mathcal{P}_{5} \mathcal{P}_{4} \mathcal{P}_{3} \mathcal{P}_{7} \mathcal{P}_{6} \mathcal{R}_{\hat{4}, 7} \mathcal{P}_{11} \mathcal{P}_{12} \mathcal{P}_{10} \mathcal{P}_{9} \mathcal{P}_{6} \mathcal{P}_{5} \mathcal{P}_{4} \mathcal{R}_{\hat{5}, 5} \mathcal{P}_{9} \mathcal{P}_{10} \mathcal{P}_{11} \mathcal{P}_{8} \\
\mathcal{R}_{\hat{6}, 1} \mathcal{P}_{5} \mathcal{P}_{6} \mathcal{P}_{7} \mathcal{P}_{8} \mathcal{P}_{9} \mathcal{P}_{10} \mathcal{P}_{4} \mathcal{P}_{5} \mathcal{P}_{6} \mathcal{P}_{3} \\
=\mathcal{P}_{12} \mathcal{P}_{9} \mathcal{P}_{10} \mathcal{P}_{11} \mathcal{P}_{8} \mathcal{P}_{5} \mathcal{P}_{6} \mathcal{P}_{7} \mathcal{P}_{8} \mathcal{P}_{9} \mathcal{P}_{10} \mathcal{P}_{4} \mathcal{P}_{5} \mathcal{P}_{6} \mathcal{P}_{3} \mathcal{R}_{\hat{6}, 11} \mathcal{R}_{\hat{5}, 7} \mathcal{P}_{11} \mathcal{P}_{12} \mathcal{P}_{13} \\
\mathcal{P}_{10} \mathcal{P}_{11} \mathcal{P}_{9} \mathcal{P}_{6} \mathcal{P}_{5} \mathcal{P}_{4} \mathcal{P}_{3} \mathcal{P}_{2} \mathcal{P}_{1} \mathcal{R}_{\hat{4}, 5} \mathcal{P}_{9} \mathcal{P}_{10} \mathcal{P}_{11} \mathcal{P}_{12} \mathcal{P}_{8} \mathcal{P}_{9} \mathcal{P}_{4} \mathcal{P}_{3} \mathcal{P}_{2} \mathcal{P}_{5} \mathcal{P}_{4} \mathcal{P}_{3} \\
\mathcal{R}_{\hat{3}, 5} \mathcal{P}_{9} \mathcal{P}_{10} \mathcal{P}_{11} \mathcal{P}_{4} \mathcal{P}_{5} \mathcal{P}_{6} \mathcal{R}_{\hat{2}, 7} \mathcal{R}_{\hat{1}, 11},
\end{aligned}
$$

see Fig. 11. The Bruhat order $B(6,3)$ is not polyhedral [38], because of the existence of "small cubes", see Remark 3.2 and Fig. 6. We can rewrite the 5 -simplex equation as follows to display the respective appearances (in brackets),

$$
\begin{aligned}
& \mathcal{R}_{\hat{1}, 1} \mathcal{R}_{\hat{2}, 5} \mathcal{P}_{\mathbf{9}} \mathcal{P}_{10}\left(\mathcal{P}_{12} \mathcal{P}_{11} \mathcal{P}_{12}\right) \mathcal{P}_{\mathbf{1 3}} \mathcal{P}_{\mathbf{1 4}} \mathcal{P}_{\mathbf{4}} \mathcal{P}_{\mathbf{3}} \mathcal{P}_{\mathbf{2}} \mathcal{P}_{\mathbf{5}} \mathcal{P}_{\mathbf{6}} \mathcal{R}_{\hat{3}, \mathbf{7}} \mathcal{P}_{\mathbf{1 1}} \mathcal{P}_{\mathbf{1 2}} \mathcal{P}_{\mathbf{1 3}} \mathcal{P}_{\mathbf{1 0}} \mathcal{P}_{11} \\
& \mathcal{P}_{6}\left(\mathcal{P}_{4} \mathcal{P}_{5} \mathcal{P}_{4}\right) \mathcal{P}_{3} \mathcal{P}_{7} \mathcal{P}_{6} \mathcal{R}_{\hat{4}, 7}\left(\mathcal{P}_{12} \mathcal{P}_{11} \mathcal{P}_{12}\right) \mathcal{P}_{10} \mathcal{P}_{9} \mathcal{P}_{6} \mathcal{P}_{5} \mathcal{P}_{4} \mathcal{R}_{\hat{5}, 5} \mathcal{P}_{9} \mathcal{P}_{10} \mathcal{P}_{11} \mathcal{R}_{\hat{6}, 1} \\
& \mathcal{P}_{5} \mathcal{P}_{6}\left(\mathcal{P}_{8} \mathcal{P}_{7} \mathcal{P}_{8}\right) \mathcal{P}_{9} \mathcal{P}_{10} \mathcal{P}_{4} \mathcal{P}_{5} \mathcal{P}_{6} \\
& =\mathcal{P}_{9} \mathcal{P}_{10} \mathcal{P}_{11} \mathcal{P}_{5} \mathcal{P}_{6}\left(\mathcal{P}_{8} \mathcal{P}_{7} \mathcal{P}_{8}\right) \mathcal{P}_{9} \mathcal{P}_{10} \mathcal{P}_{4} \mathcal{P}_{5} \mathcal{P}_{6} \mathcal{R}_{\hat{6}, 11} \mathcal{R}_{5,7} \mathcal{P}_{11} \mathcal{P}_{12} \mathcal{P}_{13} \mathcal{P}_{10} \\
& \mathcal{P}_{9} \mathcal{P}_{6} \mathcal{P}_{5}\left(\mathcal{P}_{3} \mathcal{P}_{4} \mathcal{P}_{3}\right) \mathcal{P}_{2} \mathcal{P}_{1} \mathcal{R}_{\hat{4}, 5} \mathcal{P}_{9}\left(\mathcal{P}_{11} \mathcal{P}_{10} \mathcal{P}_{11}\right) \mathcal{P}_{12} \mathcal{P}_{8} \mathcal{P}_{9} \mathcal{P}_{4} \mathcal{P}_{3} \mathcal{P}_{2} \mathcal{P}_{5} \mathcal{P}_{4} \\
& \mathcal{R}_{\hat{3}, 5} \mathcal{P}_{9} \mathcal{P}_{10} \mathcal{P}_{11}\left(\mathcal{P}_{3} \mathcal{P}_{4} \mathcal{P}_{3}\right) \mathcal{P}_{5} \mathcal{P}_{6} \mathcal{R}_{\hat{2}, 7} \mathcal{R}_{\hat{1}, 11}
\end{aligned}
$$

In terms of $\hat{\mathcal{R}}:=\mathcal{R} \mathcal{P}_{\mathbf{2 4}} \mathcal{P}_{\mathbf{1 5}}$, the 5 -simplex equation takes the form

$$
\begin{aligned}
& \hat{\mathcal{R}}_{\hat{1}, \mathbf{1 , 2 , 3 , 4 , 5}} \hat{\mathcal{R}}_{\hat{2}, \mathbf{1 , 6}, \mathbf{7}, \mathbf{8}, \mathbf{9}} \hat{\mathcal{R}}_{\hat{3}, \mathbf{2}, \mathbf{6}, 10,11,12} \hat{\mathcal{R}}_{\hat{4}, 3,7,10,13,14} \hat{\mathcal{R}}_{\overline{5}, 4,8,11,13,15} \hat{\mathcal{R}}_{\hat{6}, 5,9,12,14,15} \\
& =\hat{\mathcal{R}}_{\hat{6}, \mathbf{5}, 9,12,14,15} \hat{\mathcal{R}}_{\hat{5}, 4,8,11,13,15} \hat{\mathcal{R}}_{\hat{4}, 3,7,10,13,14} \hat{\mathcal{R}}_{\hat{3}, 2,6,10,11,12} \hat{\mathcal{R}}_{\hat{2}, 1,6,7,8,9} \hat{\mathcal{R}}_{\hat{1}, 1,2,3,4,5} .
\end{aligned}
$$

6-simplex equation. In case of $B(7,5)$ we consider maps

$$
\begin{aligned}
\mathcal{R}_{i j k l m n}: & \mathcal{U}_{i j k l m} \times \mathcal{U}_{i j k l n} \times \mathcal{U}_{i j k m n} \times \mathcal{U}_{i j l m n} \times \mathcal{U}_{i l m n} \times \mathcal{U}_{j k l m n} \\
& \longrightarrow \mathcal{U}_{j k l m n} \times \mathcal{U}_{i k l m n} \times \mathcal{U}_{i j l m n} \times \mathcal{U}_{i j k m n} \times \mathcal{U}_{i j k l n} \times \mathcal{U}_{i j k l m}
\end{aligned}
$$



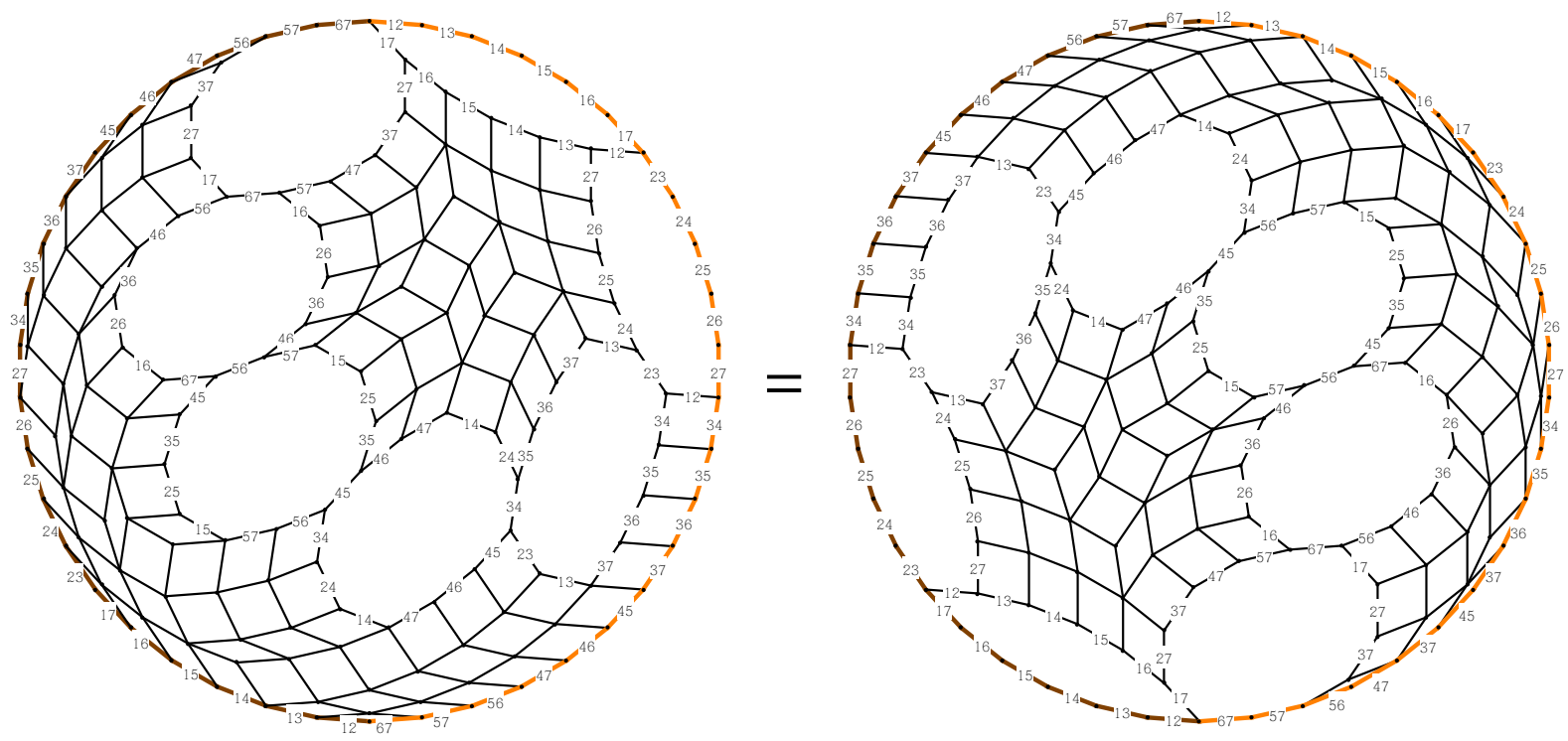

Figure 12. The two sides of the 6-simplex equation correspond to sequences of maximal chains on two complementary sides (left and right figure) of a polyhedral reduction of $B(7,4)$.

where $i<j<k<l<m<n$. Turning to complementary notation, these maps have to satisfy the 6 -simplex equation

$$
\begin{aligned}
& \mathcal{R}_{\hat{1}, 1} \mathcal{R}_{\hat{2}, 6}\left(\mathcal{P}_{11} \mathcal{P}_{12} \mathcal{P}_{13} \mathcal{P}_{14} \mathcal{P}_{15} \mathcal{P}_{\mathbf{1 6}} \mathcal{P}_{17} \mathcal{P}_{18} \mathcal{P}_{19} \mathcal{P}_{\mathbf{2 0}}\right)\left(\mathcal{P}_{\mathbf{5}} \mathcal{P}_{4} \mathcal{P}_{\mathbf{3}} \mathcal{P}_{\mathbf{2}}\right)\left(\mathcal{P}_{\mathbf{6}} \mathcal{P}_{5} \mathcal{P}_{4}\right)\left(\mathcal{P}_{\mathbf{7}} \mathcal{P}_{6}\right) \\
& \mathcal{P}_{8} \mathcal{R}_{\hat{3}, 9}\left(\mathcal{P}_{14} \mathcal{P}_{15} \mathcal{P}_{16} \mathcal{P}_{17} \mathcal{P}_{18} \mathcal{P}_{19}\right)\left(\mathcal{P}_{13} \mathcal{P}_{14} \mathcal{P}_{15} \mathcal{P}_{16} \mathcal{P}_{17} \mathcal{P}_{18}\right)\left(\mathcal{P}_{8} \mathcal{P}_{7} \mathcal{P}_{6} \mathcal{P}_{5} \mathcal{P}_{4} \mathcal{P}_{3}\right) \\
& \left(\mathcal{P}_{\mathbf{9}} \mathcal{P}_{8} \mathcal{P}_{7} \mathcal{P}_{6}\right)\left(\mathcal{P}_{10} \mathcal{P}_{9}\right) \mathcal{R}_{\hat{4}, 10}\left(\mathcal{P}_{15} \mathcal{P}_{16} \mathcal{P}_{17} \mathcal{P}_{18}\right)\left(\mathcal{P}_{14} \mathcal{P}_{15} \mathcal{P}_{16}\right)\left(\mathcal{P}_{13} \mathcal{P}_{14} \mathcal{P}_{15}\right) \\
& \left(\mathcal{P}_{\mathbf{9}} \mathcal{P}_{\mathbf{8}} \mathcal{P}_{\mathbf{7}} \mathcal{P}_{6} \mathcal{P}_{\mathbf{5}} \mathcal{P}_{4}\right)\left(\mathcal{P}_{\mathbf{1 0}} \mathcal{P}_{\mathbf{9}} \mathcal{P}_{\mathbf{8}}\right) \mathcal{R}_{\hat{5}, 9}\left(\mathcal{P}_{\mathbf{1 4}} \mathcal{P}_{\mathbf{1 5}} \mathcal{P}_{\mathbf{1 6}} \mathcal{P}_{\mathbf{1 7}}\right)\left(\mathcal{P}_{\mathbf{1 3}} \mathcal{P}_{14}\right)\left(\mathcal{P}_{\mathbf{1 2}} \mathcal{P}_{11}\right) \\
& \left(\mathcal{P}_{8} \mathcal{P}_{7} \mathcal{P}_{6} \mathcal{P}_{5}\right) \mathcal{R}_{\hat{6}, 6}\left(\mathcal{P}_{11} \mathcal{P}_{12} \mathcal{P}_{13} \mathcal{P}_{14} \mathcal{P}_{15} \mathcal{P}_{16}\right)\left(\mathcal{P}_{10} \mathcal{P}_{11} \mathcal{P}_{12}\right) \mathcal{P}_{9} \mathcal{R}_{\hat{7}, 1} \\
& \left(\mathcal{P}_{6} \mathcal{P}_{7} \mathcal{P}_{8} \mathcal{P}_{9} \mathcal{P}_{10} \mathcal{P}_{11} \mathcal{P}_{12} \mathcal{P}_{13} \mathcal{P}_{14} \mathcal{P}_{15}\right)\left(\mathcal{P}_{5} \mathcal{P}_{6} \mathcal{P}_{7} \mathcal{P}_{8} \mathcal{P}_{9} \mathcal{P}_{10}\right)\left(\mathcal{P}_{4} \mathcal{P}_{5} \mathcal{P}_{6}\right) \mathcal{P}_{3} \\
& =\mathcal{P}_{18}\left(\mathcal{P}_{15} \mathcal{P}_{16} \mathcal{P}_{17}\right) \mathcal{P}_{14}\left(\mathcal{P}_{11} \mathcal{P}_{12} \mathcal{P}_{13} \mathcal{P}_{14} \mathcal{P}_{15} \mathcal{P}_{16}\right)\left(\mathcal{P}_{10} \mathcal{P}_{11} \mathcal{P}_{12}\right) \mathcal{P}_{9} \\
& \left(\mathcal{P}_{6} \mathcal{P}_{7} \mathcal{P}_{8} \mathcal{P}_{9} \mathcal{P}_{10} \mathcal{P}_{11} \mathcal{P}_{12} \mathcal{P}_{13} \mathcal{P}_{14} \mathcal{P}_{15}\right)\left(\mathcal{P}_{5} \mathcal{P}_{6} \mathcal{P}_{7} \mathcal{P}_{8} \mathcal{P}_{9} \mathcal{P}_{10}\right)\left(\mathcal{P}_{4} \mathcal{P}_{5} \mathcal{P}_{6}\right) \mathcal{P}_{3} \mathcal{R}_{\overline{7}, 16} \mathcal{R}_{\hat{6}, 11} \\
& \left(\mathcal{P}_{16} \mathcal{P}_{17} \mathcal{P}_{18} \mathcal{P}_{19}\right)\left(\mathcal{P}_{15} \mathcal{P}_{16} \mathcal{P}_{17}\right)\left(\mathcal{P}_{14} \mathcal{P}_{15}\right) \mathcal{P}_{13}\left(\mathcal{P}_{10} \mathcal{P}_{9} \mathcal{P}_{8} \mathcal{P}_{7} \mathcal{P}_{6} \mathcal{P}_{5} \mathcal{P}_{4} \mathcal{P}_{3} \mathcal{P}_{2} \mathcal{P}_{1}\right) \mathcal{R}_{5,8} \\
& \left(\mathcal{P}_{13} \mathcal{P}_{14} \mathcal{P}_{15} \mathcal{P}_{16} \mathcal{P}_{17} \mathcal{P}_{18}\right)\left(\mathcal{P}_{12} \mathcal{P}_{13} \mathcal{P}_{14} \mathcal{P}_{15}\right)\left(\mathcal{P}_{11} \mathcal{P}_{12}\right)\left(\mathcal{P}_{7} \mathcal{P}_{6} \mathcal{P}_{5} \mathcal{P}_{4} \mathcal{P}_{3} \mathcal{P}_{2}\right) \\
& \left(\mathcal{P}_{8} \mathcal{P}_{7} \mathcal{P}_{6} \mathcal{P}_{5} \mathcal{P}_{4} \mathcal{P}_{3}\right) \mathcal{R}_{\hat{4}, 7}\left(\mathcal{P}_{12} \mathcal{P}_{13} \mathcal{P}_{14} \mathcal{P}_{15} \mathcal{P}_{16} \mathcal{P}_{17}\right)\left(\mathcal{P}_{11} \mathcal{P}_{12} \mathcal{P}_{13}\right)\left(\mathcal{P}_{6} \mathcal{P}_{5} \mathcal{P}_{4}\right)\left(\mathcal{P}_{7} \mathcal{P}_{6} \mathcal{P}_{5}\right) \\
& \left(\mathcal{P}_{\mathbf{8}} \mathcal{P}_{\mathbf{7}} \mathcal{P}_{6}\right) \mathcal{R}_{\hat{3}, 8}\left(\mathcal{P}_{13} \mathcal{P}_{14} \mathcal{P}_{15} \mathcal{P}_{16}\right) \mathcal{P}_{\mathbf{7}} \mathcal{P}_{\mathbf{8}} \mathcal{P}_{\mathbf{9}} \mathcal{P}_{10} \mathcal{R}_{\hat{2}, 11} \mathcal{R}_{\hat{1}, 16}
\end{aligned}
$$

The two sides of this equation correspond to sequences of maximal chains on complementary sides of a polyhedron, see Fig. 12. In terms of $\hat{\mathcal{R}}=\mathcal{R} \mathcal{P}_{\mathbf{3 4}} \mathcal{P}_{\mathbf{2 5}} \mathcal{P}_{\mathbf{1 6}}$, the 6 -simplex equation takes the form

$$
\begin{aligned}
& \hat{\mathcal{R}}_{\hat{1}, \mathbf{1}, 2,3,4,5,6} \hat{\mathcal{R}}_{\hat{2}, 1,7,8,9,10,11} \hat{\mathcal{R}}_{\hat{3}, 2,7,12,13,14,15} \hat{\mathcal{R}}_{\hat{4}, 3,8,12,16,17,18} \hat{\mathcal{R}}_{\hat{5}, 4,9,13,16,19,20} \\
& \hat{\mathcal{R}}_{\hat{6}, 5,10,14,17,19,21} \hat{\mathcal{R}}_{\hat{7}, 6,11,15,18,20,21} \\
& =\hat{\mathcal{R}}_{\hat{7}, 6,11,15,18,20,21} \hat{\mathcal{R}}_{\hat{6}, 5,10,14,17,19,21} \hat{\mathcal{R}}_{\hat{5}, 4,9,13,16,19,20} \hat{\mathcal{R}}_{\hat{4}, 3,8,12,16,17,18} \\
& \hat{\mathcal{R}}_{\hat{3}, \mathbf{2}, \mathbf{7}, \mathbf{1 2 , 1 3 , 1 4 , 1 5}} \hat{\mathcal{R}}_{\hat{2}, \mathbf{1 , 7}, \mathbf{8 , 9}, \mathbf{1 0 , 1 1}} \hat{\mathcal{R}}_{\hat{1}, \mathbf{1}, \mathbf{2}, \mathbf{3}, \mathbf{4}, \mathbf{5}, \mathbf{6}} \text {. }
\end{aligned}
$$


7-simplex equation. Here we consider maps

$$
\begin{aligned}
\mathcal{R}_{i j k l m n p}: & \mathcal{U}_{i j k l m n} \times \mathcal{U}_{i j k l m p} \times \mathcal{U}_{i j k n p} \times \mathcal{U}_{i j k m n p} \times \mathcal{U}_{i j l m n p} \times \mathcal{U}_{i l m n p} \times \mathcal{U}_{j k l m n p} \\
& \longrightarrow \mathcal{U}_{j k l m n p} \times \mathcal{U}_{i k l m n p} \times \mathcal{U}_{i j l m n p} \times \mathcal{U}_{i j k m n p} \times \mathcal{U}_{i j k l n p} \times \mathcal{U}_{i j k l m p} \times \mathcal{U}_{i j k l m n},
\end{aligned}
$$

where $i<j<k<l<m<n<p$. The 7 -simplex equation reads

$$
\begin{aligned}
& \mathcal{R}_{\hat{1}, 1} \mathcal{R}_{\hat{2}, 7} \mathcal{P}_{13} \mathcal{P}_{14} \mathcal{P}_{15} \mathcal{P}_{16} \mathcal{P}_{17} \mathcal{P}_{18} \mathcal{P}_{19} \mathcal{P}_{20} \mathcal{P}_{21} \mathcal{P}_{22} \mathcal{P}_{23} \mathcal{P}_{24} \mathcal{P}_{25} \mathcal{P}_{26} \mathcal{P}_{27} \mathcal{P}_{6} \mathcal{P}_{5} \mathcal{P}_{4} \mathcal{P}_{3} \\
& \mathcal{P}_{\mathbf{2}} \mathcal{P}_{7} \mathcal{P}_{6} \mathcal{P}_{5} \mathcal{P}_{4} \mathcal{P}_{8} \mathcal{P}_{7} \mathcal{P}_{6} \mathcal{P}_{9} \mathcal{P}_{8} \mathcal{P}_{10} \mathcal{R}_{\hat{3}, 11} \mathcal{P}_{17} \mathcal{P}_{18} \mathcal{P}_{19} \mathcal{P}_{20} \mathcal{P}_{21} \mathcal{P}_{22} \mathcal{P}_{23} \mathcal{P}_{24} \mathcal{P}_{25} \mathcal{P}_{26} \\
& \mathcal{P}_{16} \mathcal{P}_{17} \mathcal{P}_{18} \mathcal{P}_{19} \mathcal{P}_{20} \mathcal{P}_{21} \mathcal{P}_{22} \mathcal{P}_{23} \mathcal{P}_{24} \mathcal{P}_{25} \mathcal{P}_{10} \mathcal{P}_{9} \mathcal{P}_{8} \mathcal{P}_{7} \mathcal{P}_{6} \mathcal{P}_{5} \mathcal{P}_{4} \mathcal{P}_{3} \mathcal{P}_{11} \mathcal{P}_{10} \mathcal{P}_{9} \mathcal{P}_{8} \\
& \mathcal{P}_{7} \mathcal{P}_{6} \mathcal{P}_{12} \mathcal{P}_{11} \mathcal{P}_{10} \mathcal{P}_{9} \mathcal{P}_{13} \mathcal{P}_{12} \mathcal{R}_{\hat{4}, 13} \mathcal{P}_{19} \mathcal{P}_{20} \mathcal{P}_{21} \mathcal{P}_{22} \mathcal{P}_{23} \mathcal{P}_{24} \mathcal{P}_{25} \mathcal{P}_{18} \mathcal{P}_{19} \mathcal{P}_{20} \mathcal{P}_{21} \\
& \mathcal{P}_{22} \mathcal{P}_{23} \mathcal{P}_{17} \mathcal{P}_{18} \mathcal{P}_{19} \mathcal{P}_{20} \mathcal{P}_{21} \mathcal{P}_{22} \mathcal{P}_{12} \mathcal{P}_{11} \mathcal{P}_{10} \mathcal{P}_{9} \mathcal{P}_{8} \mathcal{P}_{7} \mathcal{P}_{6} \mathcal{P}_{5} \mathcal{P}_{4} \mathcal{P}_{13} \mathcal{P}_{12} \mathcal{P}_{11} \mathcal{P}_{10} \\
& \mathcal{P}_{9} \mathcal{P}_{8} \mathcal{P}_{14} \mathcal{P}_{13} \mathcal{P}_{12} \mathcal{R}_{\hat{5}, 13} \mathcal{P}_{19} \mathcal{P}_{20} \mathcal{P}_{21} \mathcal{P}_{22} \mathcal{P}_{23} \mathcal{P}_{24} \mathcal{P}_{18} \mathcal{P}_{19} \mathcal{P}_{20} \mathcal{P}_{21} \mathcal{P}_{17} \mathcal{P}_{18} \mathcal{P}_{19} \mathcal{P}_{16} \\
& \mathcal{P}_{17} \mathcal{P}_{18} \mathcal{P}_{12} \mathcal{P}_{11} \mathcal{P}_{10} \mathcal{P}_{9} \mathcal{P}_{8} \mathcal{P}_{7} \mathcal{P}_{6} \mathcal{P}_{5} \mathcal{P}_{13} \mathcal{P}_{12} \mathcal{P}_{11} \mathcal{P}_{10} \mathcal{R}_{6,11} \mathcal{P}_{17} \mathcal{P}_{18} \mathcal{P}_{19} \mathcal{P}_{20} \mathcal{P}_{21} \mathcal{P}_{22} \\
& \mathcal{P}_{23} \mathcal{P}_{16} \mathcal{P}_{17} \mathcal{P}_{18} \mathcal{P}_{19} \mathcal{P}_{15} \mathcal{P}_{16} \mathcal{P}_{14} \mathcal{P}_{13} \mathcal{P}_{10} \mathcal{P}_{9} \mathcal{P}_{8} \mathcal{P}_{7} \mathcal{P}_{6} \mathcal{R}_{\hat{7}, 7} \mathcal{P}_{13} \mathcal{P}_{14} \mathcal{P}_{15} \mathcal{P}_{16} \mathcal{P}_{17} \mathcal{P}_{18} \\
& \mathcal{P}_{19} \mathcal{P}_{20} \mathcal{P}_{21} \mathcal{P}_{22} \mathcal{P}_{12} \mathcal{P}_{13} \mathcal{P}_{14} \mathcal{P}_{15} \mathcal{P}_{16} \mathcal{P}_{17} \mathcal{P}_{11} \mathcal{P}_{12} \mathcal{P}_{13} \mathcal{P}_{10} \mathcal{R}_{\hat{8}, 1} \mathcal{P}_{7} \mathcal{P}_{8} \mathcal{P}_{9} \mathcal{P}_{10} \mathcal{P}_{11} \mathcal{P}_{12} \\
& \mathcal{P}_{13} \mathcal{P}_{14} \mathcal{P}_{15} \mathcal{P}_{16} \mathcal{P}_{17} \mathcal{P}_{18} \mathcal{P}_{19} \mathcal{P}_{20} \mathcal{P}_{21} \mathcal{P}_{6} \mathcal{P}_{7} \mathcal{P}_{8} \mathcal{P}_{9} \mathcal{P}_{10} \mathcal{P}_{11} \mathcal{P}_{12} \mathcal{P}_{13} \mathcal{P}_{14} \mathcal{P}_{15} \mathcal{P}_{5} \mathcal{P}_{6} \mathcal{P}_{7} \\
& \mathcal{P}_{8} \mathcal{P}_{9} \mathcal{P}_{10} \mathcal{P}_{4} \mathcal{P}_{5} \mathcal{P}_{6} \mathcal{P}_{3} \\
& =\mathcal{P}_{25} \mathcal{P}_{22} \mathcal{P}_{23} \mathcal{P}_{24} \mathcal{P}_{21} \mathcal{P}_{18} \mathcal{P}_{19} \mathcal{P}_{20} \mathcal{P}_{21} \mathcal{P}_{22} \mathcal{P}_{23} \mathcal{P}_{17} \mathcal{P}_{18} \mathcal{P}_{19} \mathcal{P}_{16} \mathcal{P}_{13} \mathcal{P}_{14} \mathcal{P}_{15} \mathcal{P}_{16} \mathcal{P}_{17} \\
& \mathcal{P}_{18} \mathcal{P}_{19} \mathcal{P}_{20} \mathcal{P}_{21} \mathcal{P}_{22} \mathcal{P}_{12} \mathcal{P}_{13} \mathcal{P}_{14} \mathcal{P}_{15} \mathcal{P}_{16} \mathcal{P}_{17} \mathcal{P}_{11} \mathcal{P}_{12} \mathcal{P}_{13} \mathcal{P}_{10} \mathcal{P}_{7} \mathcal{P}_{8} \mathcal{P}_{9} \mathcal{P}_{10} \mathcal{P}_{11} \\
& \mathcal{P}_{12} \mathcal{P}_{13} \mathcal{P}_{14} \mathcal{P}_{15} \mathcal{P}_{16} \mathcal{P}_{17} \mathcal{P}_{18} \mathcal{P}_{19} \mathcal{P}_{20} \mathcal{P}_{21} \mathcal{P}_{6} \mathcal{P}_{7} \mathcal{P}_{8} \mathcal{P}_{9} \mathcal{P}_{10} \mathcal{P}_{11} \mathcal{P}_{12} \mathcal{P}_{13} \mathcal{P}_{14} \mathcal{P}_{15} \mathcal{P}_{5} \\
& \mathcal{P}_{6} \mathcal{P}_{7} \mathcal{P}_{8} \mathcal{P}_{9} \mathcal{P}_{10} \mathcal{P}_{4} \mathcal{P}_{5} \mathcal{P}_{6} \mathcal{P}_{3} \mathcal{R}_{\hat{8}, 22} \mathcal{R}_{\overline{7}, 16} \mathcal{P}_{22} \mathcal{P}_{23} \mathcal{P}_{24} \mathcal{P}_{25} \mathcal{P}_{26} \mathcal{P}_{21} \mathcal{P}_{22} \mathcal{P}_{23} \mathcal{P}_{24} \mathcal{P}_{20} \\
& \mathcal{P}_{21} \mathcal{P}_{22} \mathcal{P}_{19} \mathcal{P}_{20} \mathcal{P}_{18} \mathcal{P}_{15} \mathcal{P}_{14} \mathcal{P}_{13} \mathcal{P}_{12} \mathcal{P}_{11} \mathcal{P}_{10} \mathcal{P}_{9} \mathcal{P}_{8} \mathcal{P}_{7} \mathcal{P}_{6} \mathcal{P}_{5} \mathcal{P}_{4} \mathcal{P}_{3} \mathcal{P}_{2} \mathcal{P}_{1} \mathcal{R}_{\hat{6}, 12} \mathcal{P}_{18} \\
& \mathcal{P}_{19} \mathcal{P}_{20} \mathcal{P}_{21} \mathcal{P}_{22} \mathcal{P}_{23} \mathcal{P}_{24} \mathcal{P}_{25} \mathcal{P}_{17} \mathcal{P}_{18} \mathcal{P}_{19} \mathcal{P}_{20} \mathcal{P}_{21} \mathcal{P}_{22} \mathcal{P}_{16} \mathcal{P}_{17} \mathcal{P}_{18} \mathcal{P}_{19} \mathcal{P}_{15} \mathcal{P}_{16} \mathcal{P}_{11} \\
& \mathcal{P}_{10} \mathcal{P}_{9} \mathcal{P}_{8} \mathcal{P}_{7} \mathcal{P}_{6} \mathcal{P}_{5} \mathcal{P}_{4} \mathcal{P}_{3} \mathcal{P}_{2} \mathcal{P}_{12} \mathcal{P}_{11} \mathcal{P}_{10} \mathcal{P}_{9} \mathcal{P}_{8} \mathcal{P}_{7} \mathcal{P}_{6} \mathcal{P}_{5} \mathcal{P}_{4} \mathcal{P}_{3} \mathcal{R}_{5,10} \mathcal{P}_{16} \mathcal{P}_{17} \mathcal{P}_{18} \\
& \mathcal{P}_{19} \mathcal{P}_{20} \mathcal{P}_{21} \mathcal{P}_{22} \mathcal{P}_{23} \mathcal{P}_{24} \mathcal{P}_{15} \mathcal{P}_{16} \mathcal{P}_{17} \mathcal{P}_{18} \mathcal{P}_{19} \mathcal{P}_{20} \mathcal{P}_{14} \mathcal{P}_{15} \mathcal{P}_{16} \mathcal{P}_{9} \mathcal{P}_{8} \mathcal{P}_{7} \mathcal{P}_{6} \mathcal{P}_{5} \mathcal{P}_{4} \\
& \mathcal{P}_{10} \mathcal{P}_{9} \mathcal{P}_{8} \mathcal{P}_{7} \mathcal{P}_{6} \mathcal{P}_{5} \mathcal{P}_{11} \mathcal{P}_{10} \mathcal{P}_{9} \mathcal{P}_{8} \mathcal{P}_{7} \mathcal{P}_{6} \mathcal{R}_{\hat{4}, 10} \mathcal{P}_{16} \mathcal{P}_{17} \mathcal{P}_{18} \mathcal{P}_{19} \mathcal{P}_{20} \mathcal{P}_{21} \mathcal{P}_{22} \mathcal{P}_{23} \\
& \mathcal{P}_{15} \mathcal{P}_{16} \mathcal{P}_{17} \mathcal{P}_{18} \mathcal{P}_{9} \mathcal{P}_{8} \mathcal{P}_{7} \mathcal{P}_{10} \mathcal{P}_{9} \mathcal{P}_{8} \mathcal{P}_{11} \mathcal{P}_{10} \mathcal{P}_{9} \mathcal{P}_{12} \mathcal{P}_{11} \mathcal{P}_{10} \mathcal{R}_{\hat{3}, 12} \mathcal{P}_{18} \mathcal{P}_{19} \mathcal{P}_{20} \mathcal{P}_{21} \\
& \mathcal{P}_{22} \mathcal{P}_{11} \mathcal{P}_{12} \mathcal{P}_{13} \mathcal{P}_{14} \mathcal{P}_{15} \mathcal{R}_{\hat{2}, 16} \mathcal{R}_{\hat{1}, 22} \text {. }
\end{aligned}
$$

Also see Fig. 13. In terms of $\hat{\mathcal{R}}=\mathcal{R} \mathcal{P}_{\mathbf{3 5}} \mathcal{P}_{\mathbf{2 6}} \mathcal{P}_{\mathbf{1 7}}$, it collapses to

$$
\begin{aligned}
& \hat{\mathcal{R}}_{\hat{1}, 1,2,3,4,5,6,7} \hat{\mathcal{R}}_{\hat{2}, 1,8,9,10,11,12,13} \hat{\mathcal{R}}_{\hat{3}, 2,8,14,15,16,17,18} \hat{\mathcal{R}}_{\hat{4}, 3,9,14,19,20,21,22} \\
& \hat{\mathcal{R}}_{\hat{5}, 4,10,15,19,23,24,25} \hat{\mathcal{R}}_{\hat{6}, 5,11,16,20,23,26,27} \hat{\mathcal{R}}_{\hat{7}, 6,12,17,21,24,26,28} \hat{\mathcal{R}}_{\hat{8}, 7,13,18,22,25,27,28} \\
& =\hat{\mathcal{R}}_{\hat{8}, 7,13,18,22,25,27,28} \hat{\mathcal{R}}_{\hat{7}, 6,12,17,21,24,26,28} \hat{\mathcal{R}}_{\hat{6}, 5,11,16,20,23,26,27} \hat{\mathcal{R}}_{\hat{5}, 4,10,15,19,23,24,25} \\
& \hat{\mathcal{R}}_{\hat{4}, \mathbf{3}, \mathbf{9}, \mathbf{1 4 , 1 9 , 2 0 , 2 1 , 2 2}} \hat{\mathcal{R}}_{\hat{3}, \mathbf{2}, \mathbf{8}, \mathbf{1 4 , 1 5 , 1 6 , 1 7 , 1 8}} \hat{\mathcal{R}}_{\hat{2}, \mathbf{1}, \mathbf{8}, \mathbf{9}, 10,11,12,13} \hat{\mathcal{R}}_{\hat{1}, \mathbf{1}, \mathbf{2}, \mathbf{3}, \mathbf{4}, \mathbf{5}, \mathbf{6}, \mathbf{7}} .
\end{aligned}
$$

Remark 3.4. The form in which the above zonohedra appear, i.e., decomposed in two complementary parts, reveals an interesting feature. If we identify antipodal edges (carrying the same label) of the boundaries, in each of the two parts, we obtain the same projective polyhedron. ${ }^{13}$ From the cube, associated with the Yang-Baxter equation, we obtain in this way two copies of the hemicube. From the permutahedron, associated with the Zamolodchikov equation, we

\footnotetext{
${ }^{13}$ Corresponding resolutions of small cubes have to be chosen.
} 

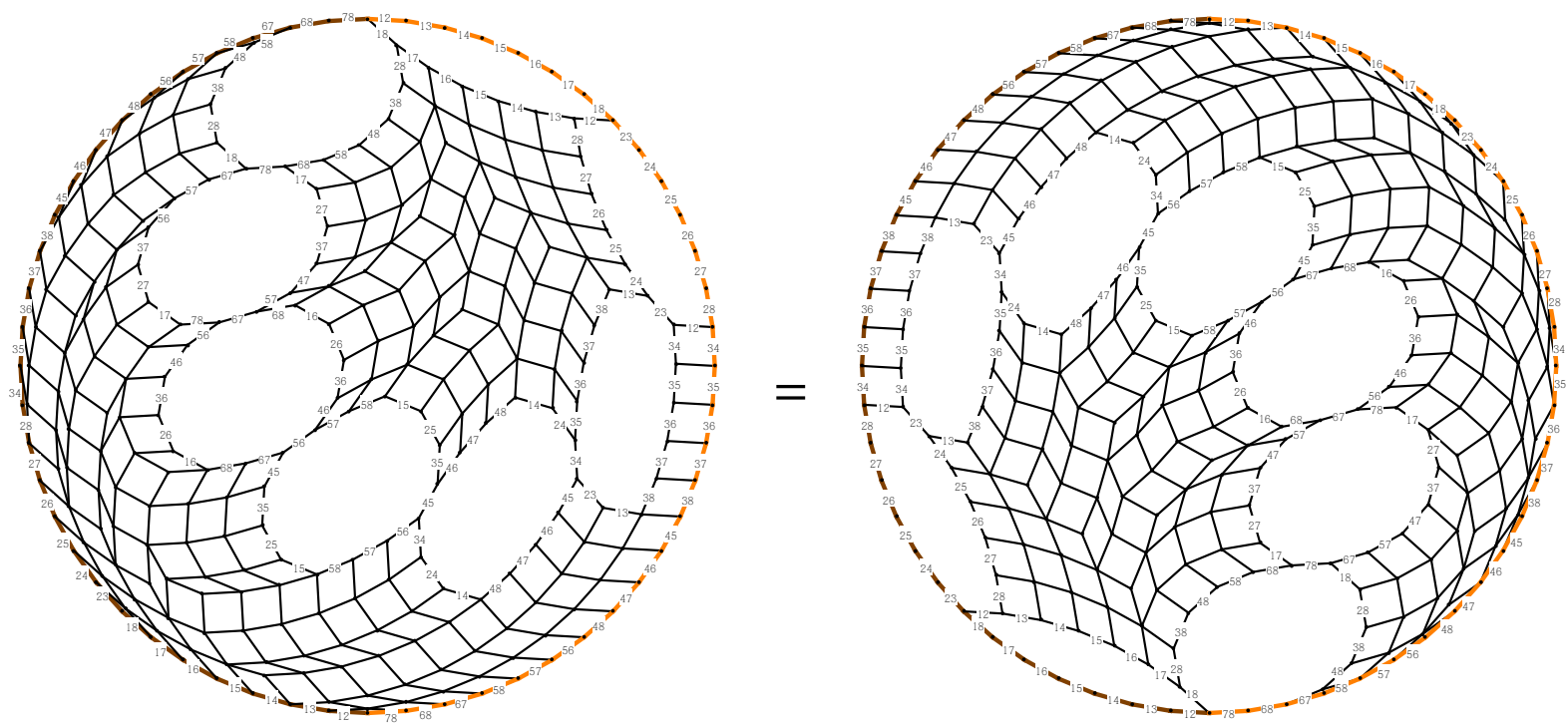

Figure 13. Two complementary sides of a polyhedral reduction of $B(8,5)$. The 7 -simplex equation corresponds to sequences of maximal chains on them.

obtain two copies of a "hemi-permutahedron". Here the identification of antipodal edges means identification of a permutation with the corresponding reversed permutation, e.g., $1234 \cong 4321$, $1324 \cong 4231$.

\subsection{Lax systems for simplex equations}

We promote the maps $\mathcal{R}_{J}: \mathcal{U}_{\vec{P}(J)} \rightarrow \mathcal{U}_{\overleftarrow{P}(J)}, J \in\left(\begin{array}{c}{[N+2]} \\ N\end{array}\right)$, to "localized" maps

$$
\begin{aligned}
\mathcal{L}_{J}: \mathcal{U}_{J} & \longrightarrow \operatorname{Map}\left(\mathcal{U}_{\vec{P}(J)}, \mathcal{U}_{\overleftarrow{P}(J)}\right) \\
u_{J} & \longmapsto \mathcal{L}_{J}\left(u_{J}\right): \mathcal{U}_{\vec{P}(J)} \rightarrow \mathcal{U}_{\overleftarrow{P}(J)} .
\end{aligned}
$$

In $B(N+2, N-1)$, counterparts of the two maximal chains, of which $B(N+1, N-1)$ consists, appear as chains for all $\hat{k}=[N+2] \backslash\{k\}=\left\{k_{1}, \ldots, k_{N+1}\right\} \in\left(\begin{array}{c}{[N+2]} \\ N+1\end{array}\right)$ :

$$
\begin{aligned}
& \mathcal{C}_{\hat{k}, \text { lex }}:\left[\alpha_{\hat{k}}\right] \stackrel{\widehat{k k_{N+1}}}{\longrightarrow}\left[\rho_{\hat{k}, 1}\right] \stackrel{\widehat{k k_{N}}}{\longrightarrow} \ldots \stackrel{\widehat{k k_{2}}}{\longrightarrow}\left[\rho_{\hat{k}, N}\right] \stackrel{\widehat{k k_{1}}}{\longrightarrow}\left[\omega_{\hat{k}}\right], \\
& \mathcal{C}_{\hat{k}, \text { rev }}:\left[\alpha_{\hat{k}}\right] \stackrel{\widehat{k k_{1}}}{\longrightarrow}\left[\sigma_{\hat{k}, 1}\right] \stackrel{\widehat{k k_{2}}}{\longrightarrow} \ldots \stackrel{\widehat{k k_{N}}}{\longrightarrow}\left[\sigma_{\hat{k}, N}\right] \stackrel{\widehat{k k_{N+1}}}{\longrightarrow}\left[\omega_{\hat{k}}\right],
\end{aligned}
$$

where $\alpha_{\hat{k}}, \omega_{\hat{k}}, \rho_{\hat{k}, i}, \sigma_{\hat{k}, i}$ are admissible linear orders of $\left(\begin{array}{c}\hat{k} \\ N-1\end{array}\right)$, the $k_{i}$ are assumed to be in natural order, and $\widehat{k k}_{i}=\hat{k} \backslash\left\{k_{i}\right\}=[N+2] \backslash\left\{k, k_{i}\right\}$. For each $k \in[N+2]$, we then impose the localized $N$-simplex equation,

$$
\mathcal{L}_{\tilde{\mathcal{C}}_{\hat{k}, \text { lex }}}\left(\boldsymbol{u}_{\vec{P}(\hat{k})}\right)=\mathcal{L}_{\tilde{\mathcal{C}}_{\hat{k}, \mathrm{rev}}}\left(\boldsymbol{v}_{\overleftarrow{P}(\hat{k})}\right)
$$

with $\boldsymbol{u}_{\vec{P}(\hat{k})}=\left(u_{\widehat{k k_{N+1}}}, \ldots, u_{\widehat{k k_{1}}}\right)$ and $\boldsymbol{v}_{\overleftarrow{P}(\hat{k})}=\left(v_{\widehat{k k_{1}}}, \ldots, v_{\widehat{k k_{N+1}}}\right)$. We assume that, for each $k \in[N+2]$, this equation uniquely determines a map $\mathcal{R}_{\hat{k}}: \boldsymbol{u}_{\vec{P}(\hat{k})} \mapsto \boldsymbol{v}_{\overleftarrow{P}(\hat{k})} \cdot$

In terms of

$$
\hat{\mathcal{L}}_{\widehat{k k_{i}}}:=\mathcal{L}_{\widehat{k k_{i}}} \mathcal{P}_{\widehat{k k_{i}}}
$$


the above equation has the form

$$
\hat{\mathcal{L}}_{\widehat{k k_{1}}, \boldsymbol{X}_{\boldsymbol{A}_{1}}} \hat{\mathcal{L}}_{\widehat{k k_{2}}, \boldsymbol{X}_{\boldsymbol{A}_{2}}} \cdots \hat{\mathcal{L}}_{\widehat{k k_{N+1}}, \boldsymbol{X}_{\boldsymbol{A}_{N+1}}}=\left(\hat{\mathcal{L}}_{k k_{N+1}}, \boldsymbol{X}_{\boldsymbol{A}_{N+1}} \cdots \hat{\mathcal{L}}_{\widehat{k k_{2}}, \boldsymbol{X}_{\boldsymbol{A}_{2}}} \hat{\mathcal{L}}_{\widehat{k k_{1}}, \boldsymbol{X}_{\boldsymbol{A}_{1}}}\right) \circ \mathcal{R}_{\hat{k}} .
$$

Here $\boldsymbol{X}_{\boldsymbol{A}_{i}}=\left(\boldsymbol{x}_{\boldsymbol{a}_{i, 1}}, \ldots, \boldsymbol{x}_{\boldsymbol{a}_{i, N+1}}\right)$, where $1 \leq \boldsymbol{x}_{\boldsymbol{a}_{i, j}} \leq c(N+2, N-1)$, are increasing sequences of positive integers and $\boldsymbol{A}_{i}=\left(\boldsymbol{a}_{i, 1}, \ldots, \boldsymbol{a}_{i, N+1}\right)$, where $1 \leq \boldsymbol{a}_{i, j} \leq c(N+1, N-1)$, are the multi-indices introduced in Remark 3.3 .

With $\rho=\left(J_{1}, \ldots, J_{c(N+2, N)}\right) \in A(N+2, N)$, we associate the composition

$$
\hat{\mathcal{L}}_{\rho}=\hat{\mathcal{L}}_{J_{c(N+2, N)}, \boldsymbol{A}_{c(N+2, N)}} \cdots \hat{\mathcal{L}}_{J_{1}, \boldsymbol{A}_{1}}: \mathcal{U}_{\eta} \rightarrow \mathcal{U}_{\eta}
$$

of the corresponding maps. Here $\eta \in A(N+2, N-1)$ is the reverse lexicographical order of $\left(\begin{array}{c}{[N+2]} \\ N-1\end{array}\right)$, and the multi-indices $\boldsymbol{A}_{i}$ specify the positions of the elements of $P\left(J_{i}\right)$ in $\eta$. The next observation will be important in the following.

Lemma 3.5. If $J, J^{\prime} \in\left(\begin{array}{c}{[N+2]} \\ N\end{array}\right)$ satisfy $E(J) \cap E\left(J^{\prime}\right)=\varnothing$, then $\hat{\mathcal{L}}_{J, \boldsymbol{A}} \hat{\mathcal{L}}_{J^{\prime}, \boldsymbol{A}^{\prime}}=\hat{\mathcal{L}}_{J^{\prime}, \boldsymbol{A}^{\prime}} \hat{\mathcal{L}}_{J, \boldsymbol{A}}($ acting on some $\left.\mathcal{U}_{\mu}, \mu \in A(N+2, N-1)\right)$.

Proof. It is easily verified that $E(J) \cap E\left(J^{\prime}\right)=\varnothing \Longleftrightarrow P(J) \cap P\left(J^{\prime}\right)=\varnothing$. Hence, if $E(J) \cap$ $E\left(J^{\prime}\right)=\varnothing$, then $\hat{\mathcal{L}}_{J, \boldsymbol{A}}$ and $\hat{\mathcal{L}}_{J^{\prime}, \boldsymbol{A}^{\prime}}$ must act on distinct positions in $\mathcal{U}_{\mu}$, hence they commute.

Now we sketch a proof of the claim that the $(N+1)$-simplex equation

$$
\mathcal{R}_{\tilde{\mathcal{C}}_{\text {lex }}}=\mathcal{R}_{\tilde{\mathcal{C}}_{\text {rev }}},
$$

where $\tilde{\mathcal{C}}_{\text {lex }}$ and $\tilde{\mathcal{C}}_{\text {rev }}$ constitute a resolution of $B(N+2, N)$, arises as a consistency condition of the above Lax system. We start with $\hat{\mathcal{L}}_{\alpha}$, where $\alpha \in A(N+2, N)$ is the lexicographical order of $\left(\begin{array}{c}{[N+2]} \\ N\end{array}\right)$, and proceed according to the resolution $\tilde{\mathcal{C}}_{\text {lex }}$. The above Lemma guarantees that there is a permutation of $\hat{\mathcal{L}}$ 's, corresponding to the resolution of $[\alpha]$ that leads to $\mathcal{U}_{\rho_{0}}=\mathcal{P}_{\rho_{0}, \alpha} \mathcal{U}_{\alpha}$, which arranges that $\hat{\mathcal{L}}_{\vec{P}(\widehat{N+2})}$ acts on $\mathcal{U}_{\rho_{0}}$ at consecutive positions. This yields $\hat{\mathcal{L}}_{\rho_{0}} \circ \mathcal{P}_{\rho_{0}, \alpha}$. Next we apply the respective Lax equation (3.10), which results in $\hat{\mathcal{L}}_{\rho_{0}^{\prime}} \circ\left(\mathcal{R}_{\widehat{N+2}, \boldsymbol{a}_{0}} \mathcal{P}_{\rho_{0}, \alpha}\right)$. Proceeding in this way, we finally arrive at $\hat{\mathcal{L}}_{\omega} \circ \mathcal{R}_{\tilde{\mathcal{C}}_{\text {lex }}}$. Starting again with $\hat{\mathcal{L}}_{\alpha}$, but now following the resolution $\tilde{\mathcal{C}}_{\text {rev }}$, we finally obtain $\hat{\mathcal{L}}_{\omega} \circ \mathcal{R}_{\tilde{\mathcal{C}}_{\text {rev }}}$. Since we assumed that the Lax equations uniquely determine the respective maps, we can conclude that the $(N+1)$-simplex equation holds.

Example 3.6. Let $N=3$. Then $\alpha=(\widehat{45}, \widehat{35}, \widehat{34}, \widehat{25}, \widehat{24}, \widehat{23}, \widehat{15}, \widehat{14}, \widehat{13}, \widehat{12}) \in A(5,3)$ and $\eta=$ $(45,35,34,25,24,23,15,14,13,12) \in A(5,2)$, from which we can read off the position indices to obtain

$$
\hat{\mathcal{L}}_{\alpha}=\hat{\mathcal{L}}_{\widehat{12}, \mathbf{1 2 3}} \hat{\mathcal{L}}_{\widehat{13}, \mathbf{1 4 5}} \hat{\mathcal{L}}_{\widehat{14}, \mathbf{2 4 6}} \hat{\mathcal{L}}_{\widehat{15}, \mathbf{3 5 6}} \hat{\mathcal{L}}_{\widehat{23}, \mathbf{1 7 8}} \hat{\mathcal{L}}_{\widehat{24}, \mathbf{2 7 9}} \hat{\mathcal{L}}_{\widehat{25}, \mathbf{3 8 9}} \hat{\mathcal{L}}_{\widehat{34}, \mathbf{4 7 0}} \hat{\mathcal{L}}_{\widehat{35}, \mathbf{5 8 0}} \hat{\mathcal{L}}_{\widehat{45}, \mathbf{6 9 0}} .
$$

The Lax system takes the form

$$
\begin{aligned}
& \hat{\mathcal{L}}_{\widehat{k k_{1}}, \boldsymbol{x}_{1}, \boldsymbol{x}_{2}, \boldsymbol{x}_{\mathbf{3}}} \hat{\mathcal{L}}_{\widehat{k k_{2}}, \boldsymbol{x}_{\mathbf{1}}, \boldsymbol{x}_{\mathbf{4}}, \boldsymbol{x}_{5}} \hat{\mathcal{L}}_{\widehat{k k_{3}}, \boldsymbol{x}_{\mathbf{2}}, \boldsymbol{x}_{\mathbf{4}}, \boldsymbol{x}_{\mathbf{6}}} \hat{\mathcal{L}}_{\widehat{k k_{4}}, \boldsymbol{x}_{\mathbf{3}}, \boldsymbol{x}_{\mathbf{5}}, \boldsymbol{x}_{\mathbf{6}}}
\end{aligned}
$$

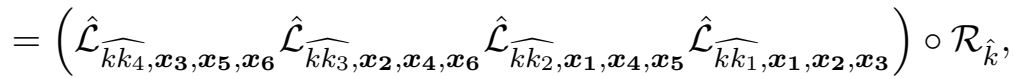

where $1 \leq \boldsymbol{x}_{1}<\boldsymbol{x}_{2}<\cdots<\boldsymbol{x}_{6} \leq 10$. Now we have

$$
\begin{aligned}
& \hat{\mathcal{L}}_{\alpha}=\hat{\mathcal{L}}_{\widehat{12}, \mathbf{1 2 3}} \hat{\mathcal{L}}_{\widehat{13}, \mathbf{1 4 5}} \hat{\mathcal{L}}_{\widehat{14}, \mathbf{2 4 6}} \hat{\mathcal{L}}_{\widehat{15}, \mathbf{3 5 6}} \hat{\mathcal{L}}_{\widehat{23}, \mathbf{1 7 8}} \hat{\mathcal{L}}_{\widehat{24}, \mathbf{2 7 9}} \hat{\mathcal{L}}_{\widehat{25}, \mathbf{3 8 9}} \hat{\mathcal{L}}_{\widehat{34}, \mathbf{4 7 0}} \hat{\mathcal{L}}_{\widehat{35}, 580} \hat{\mathcal{L}}_{\widehat{45}, 690}
\end{aligned}
$$

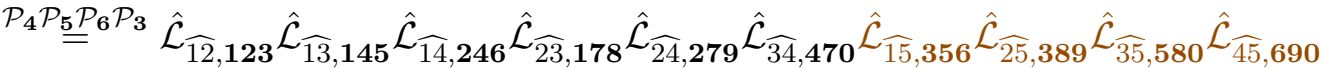




$$
\begin{aligned}
& \stackrel{\mathcal{R}_{\hat{5}, \mathbf{1}}}{=} \hat{\mathcal{L}}_{\widehat{12}, \mathbf{1 2 3}} \hat{\mathcal{L}}_{\widehat{13}, \mathbf{1 4 5}} \hat{\mathcal{L}}_{\widehat{14}, \mathbf{2 4 6}} \hat{\mathcal{L}}_{\widehat{23,178}} \hat{\mathcal{L}}_{\widehat{24}, \mathbf{2 7 9}} \hat{\mathcal{L}}_{\widehat{34}, 470} \hat{\mathcal{L}}_{\widehat{45}, \mathbf{6 9 0}} \hat{\mathcal{L}}_{\widehat{35}, \mathbf{5 8 0}} \hat{\mathcal{L}}_{\widehat{25}, \mathbf{3 8 9}} \hat{\mathcal{L}}_{\widehat{15}, \mathbf{3 5 6}} \\
& \stackrel{\mathcal{P}_{\mathbf{7}}}{=} \hat{\mathcal{L}}_{\widehat{12}, \mathbf{1 2 3}} \hat{\mathcal{L}}_{\widehat{13}, \mathbf{1 4 5}} \hat{\mathcal{L}}_{\widehat{23}, \mathbf{1 7 8}} \hat{\mathcal{L}}_{\widehat{14}, \mathbf{2 4 6}} \hat{\mathcal{L}}_{\overparen{24}, \mathbf{2 7 9}} \hat{\mathcal{L}}_{\overparen{34}, \mathbf{4 7 0}} \hat{\mathcal{L}}_{\overparen{45}, \mathbf{6 9 0}} \hat{\mathcal{L}}_{\widehat{35}, \mathbf{5 8 0}} \hat{\mathcal{L}}_{\overparen{25}, \mathbf{3 8 9}} \hat{\mathcal{L}}_{\widehat{15}, \mathbf{3 5 6}} \\
& \stackrel{\mathcal{R}_{\widehat{A}, \mathbf{4}}}{=} \hat{\mathcal{L}}_{\widehat{12}, \mathbf{1 2 3}} \hat{\mathcal{L}}_{\widehat{13}, \mathbf{1 4 5}} \hat{\mathcal{L}}_{\widehat{23}, \mathbf{1 7 8}} \hat{\mathcal{L}}_{\widehat{45}, \mathbf{6 9 0}} \hat{\mathcal{L}}_{\widehat{34,470}} \hat{\mathcal{L}}_{\widehat{24}, \mathbf{2 7 9}} \hat{\mathcal{L}}_{\widehat{14}, \mathbf{2 4 6}} \hat{\mathcal{L}}_{\widehat{35}, 580} \hat{\mathcal{L}}_{\widehat{25}, \mathbf{3 8 9}} \hat{\mathcal{L}}_{\widehat{15}, \mathbf{3 5 6}}
\end{aligned}
$$

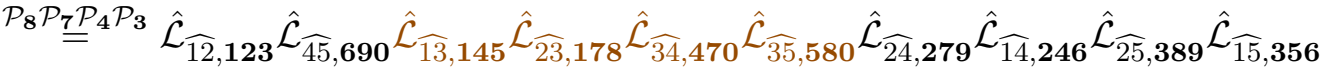

$$
\begin{aligned}
& \stackrel{\mathcal{R}_{\widehat{3}, \mathbf{5}}}{=} \hat{\mathcal{L}}_{\widehat{12}, \mathbf{1 2 3}} \hat{\mathcal{L}}_{\widehat{45}, \mathbf{6 9 0}} \hat{\mathcal{L}}_{\widehat{35}, \mathbf{5 8 0}} \hat{\mathcal{L}}_{\widehat{34}, \mathbf{4 7 0}} \hat{\mathcal{L}}_{\widehat{23,}, \mathbf{1 7 8}} \hat{\mathcal{L}}_{\widehat{13}, \mathbf{1 4 5}} \hat{\mathcal{L}}_{\widehat{24}, \mathbf{2 7 9}} \hat{\mathcal{L}}_{\widehat{14}, \mathbf{2 4 6}} \hat{\mathcal{L}}_{\widehat{25}, \mathbf{3 8 9}} \hat{\mathcal{L}}_{\widehat{15}, \mathbf{3 5 6}} \\
& \mathcal{P}_{\mathbf{7}} \mathcal{P}_{\mathbf{8}} \mathcal{P}_{\mathbf{9}} \mathcal{P}_{\mathbf{3}} \mathcal{P}_{\mathbf{2}} \mathcal{P}_{\mathbf{4}} \hat{\mathcal{L}}_{\widehat{45}, \mathbf{6 9 0}} \hat{\mathcal{L}}_{\widehat{35}, \mathbf{5 8 0}} \hat{\mathcal{L}}_{\overparen{34}, \mathbf{4 7 0}} \hat{\mathcal{L}}_{\widehat{12}, \mathbf{1 2 3}} \hat{\mathcal{L}}_{\overparen{23,178}} \hat{\mathcal{L}}_{\overparen{24}, \mathbf{2 7 9}} \hat{\mathcal{L}}_{\overparen{25}, \mathbf{3 8 9}} \hat{\mathcal{L}}_{\widehat{13}, \mathbf{1 4 5}} \hat{\mathcal{L}}_{\widehat{14}, \mathbf{2 4 6}} \hat{\mathcal{L}}_{\widehat{15}, \mathbf{3 5 6}}
\end{aligned}
$$

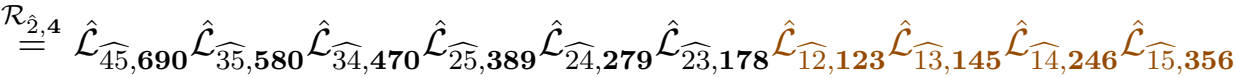

$$
\begin{aligned}
& \stackrel{\mathcal{R}_{\hat{1}} \mathbf{1}}{=} \hat{\mathcal{L}}_{\overparen{45}, \mathbf{6 9 0}} \hat{\mathcal{L}}_{\overparen{35}, \mathbf{5 8 0}} \hat{\mathcal{L}}_{\overparen{34,470}} \hat{\mathcal{L}}_{\widehat{25}, \mathbf{3 8 9}} \hat{\mathcal{L}}_{\overparen{24}, \mathbf{2 7 9}} \hat{\mathcal{L}}_{\widehat{23}, \mathbf{1 7 8}} \hat{\mathcal{L}}_{\widehat{15}, \mathbf{3 5 6}} \hat{\mathcal{L}}_{\overparen{14}, \mathbf{2 4 6}} \hat{\mathcal{L}}_{\overparen{13}, \mathbf{1 4 5}} \hat{\mathcal{L}}_{\overparen{12}, \mathbf{1 2 3}},
\end{aligned}
$$

where an index $\mathbf{0}$ stands for $\mathbf{1 0}$ (ten). Here we indicated over the equality signs the maps that act on the arguments of the $\hat{\mathcal{L}}$ 's in the respective transformation step. Returning to the proper notation, the result is $\hat{\mathcal{L}}_{\alpha}=\hat{\mathcal{L}}_{\omega} \circ \mathcal{R}_{\tilde{\mathcal{C}}_{\text {lex }}}$, which determines the left hand side of the 4-simplex equation (3.6). We marked in brown those $\hat{\mathcal{L}}$ 's that have to be brought together in order to allow for an application of a Lax equation. We could have omitted all the position indices in the above computation, since they are automatically compatible according to Lemma 3.5. But we kept them for comparison with corresponding computations in the literature (see, e.g., [44, 73, $74,75,77,82]$ ), where only these position indices appear, but not the "combinatorial indices" that nicely guided us through the above computation.

\subsection{Reductions of simplex equations}

The relation between the Bruhat order $B(N+1, N-1)$ and the $N$-simplex equation, together with the projection of Bruhat orders, defined in Remark 2.5, induces a relation between neighboring simplex equations:

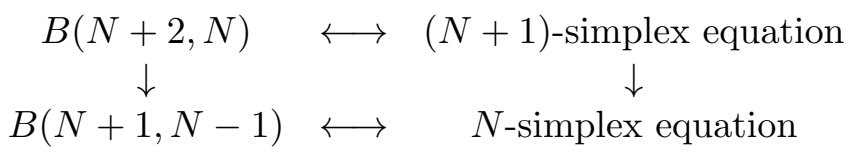

Since the structure of the $N$-simplex equation can be read off in full detail from $B(N+1, N-2)$, we shall consider the projection $B(N+2, N-1) \rightarrow B(N+1, N-2)$. Let us choose $k=N+2$ in Remark 2.5. Then all vertices of $B(N+2, N-1)$ connected by edges labeled by $\widehat{j, N+2}$ (in complementary notation), with some $j<N+2$, are identified under the projection.

For the example of $B(4,1)$ and $k=4$, the projection is shown in Fig. 3. The 3-simplex equation

$$
\hat{\mathcal{R}}_{\hat{1}, \mathbf{1 2 3}}^{(3)} \hat{\mathcal{R}}_{\hat{2}, \mathbf{1 4 5}}^{(3)} \hat{\mathcal{R}}_{\hat{3}, \mathbf{2 4 6}}^{(3)} \hat{\mathcal{R}}_{\hat{4}, \mathbf{3 5 6}}^{(3)}=\hat{\mathcal{R}}_{\hat{4}, \mathbf{3 5 6}}^{(3)} \hat{\mathcal{R}}_{\hat{3}, \mathbf{2 4 6}}^{(3)} \hat{\mathcal{R}}_{\hat{2}, \mathbf{1 4 5}}^{(3)} \hat{\mathcal{R}}_{\hat{1}, \mathbf{1 2 3}}^{(3)}
$$

acts on $\mathcal{U}_{12} \times \mathcal{U}_{13} \times \mathcal{U}_{14} \times \mathcal{U}_{23} \times \mathcal{U}_{24} \times \mathcal{U}_{34}$. The projection formally reduces this to the 2-simplex equation

$$
\hat{\mathcal{R}}_{\hat{1}, \mathbf{1 2}}^{(2)} \hat{\mathcal{R}}_{\hat{2}, \mathbf{1 3}}^{(2)} \hat{\mathcal{R}}_{\hat{3}, \mathbf{2 3}}^{(2)}=\hat{\mathcal{R}}_{\hat{3}, \mathbf{2 3}}^{(2)} \hat{\mathcal{R}}_{\hat{2}, \mathbf{1 3}}^{(2)} \hat{\mathcal{R}}_{\hat{1}, \mathbf{1 2}}^{(2)},
$$

acting on $\mathcal{U}_{12} \times \mathcal{U}_{13} \times \mathcal{U}_{23}$. Of course, in the two equations we are dealing with different types of maps (indicated by a superscript ${ }^{(2)}$, respectively ${ }^{(3)}$ ). The following relation holds. 
Proposition 3.7. Let $\hat{\mathcal{R}}^{(N)}$ satisfy the $N$-simplex equation. Let $\mathcal{U}_{\widehat{j, N+2}}, j=1,2, \ldots, N+1$, be sets, $f_{j}: \mathcal{U}_{j, \widehat{N+2}} \rightarrow \mathcal{U}_{\widehat{j, N+2}}$ and $\hat{\mathcal{R}}_{\widehat{N+2}}^{(N+1)}: \mathcal{U}_{1, \widehat{N+2}} \times \mathcal{U}_{2, \widehat{N+2}} \times \cdots \times \mathcal{U}_{N \widehat{N+1, N+2}} \rightarrow \mathcal{U}_{1, \widehat{N+2}} \times \mathcal{U}_{2, \widehat{N+2}} \times$ $\cdots \times \mathcal{U}_{N+1, N+2}$ any maps such that

$$
\left(f_{1} \times f_{2} \times \cdots \times f_{N+1}\right) \hat{\mathcal{R}}_{\widehat{N+2}}^{(N+1)}=\hat{\mathcal{R}}_{\widehat{N+2}}^{(N+1)}\left(f_{1} \times f_{2} \times \cdots \times f_{N+1}\right)
$$

holds. Setting

$$
\hat{\mathcal{R}}_{\hat{j}}^{(N+1)}:=\hat{\mathcal{R}}_{\hat{j}}^{(N)} \times f_{j}, \quad j=1,2, \ldots, N+1,
$$

then yields a solution $\hat{\mathcal{R}}_{\hat{j}}^{(N+1)}, j=1, \ldots, N+2$, of the $(N+1)$-simplex equation.

Proof. The statement can be easily verified by a direct computation.

In particular, if the maps $f_{j}, j=1,2, \ldots, N+1$, are identity functions, then the condition in the proposition is trivially satisfied and the new map defined in terms of the $N$-simplex map solves the $(N+1)$-simplex equation.

\section{Polygon equations}

In this section we address realizations of Tamari orders $T(N, n)$ in terms of sets and maps between Cartesian ${ }^{14}$ products of these sets. After some preparations in Section 4.1, polygon equations will be introduced in Section 4.2, which contains explicit expressions up to the 11gon equation, and the associated polyhedra. Section 4.3 discusses the integrability of polygon equations. Reductions of polygon equations associated with reductions of Tamari orders are the subject of Section 4.4. In the following, we write $\bar{\rho}$ instead of $\rho^{(b)}$, for an admissible linear order $\rho$.

\subsection{Resolutions of $T(N, N-2)$ and polyhedra}

The Tamari order $T(N, N-2)$ consists of the two maximal chains

$$
\begin{aligned}
& \mathcal{C}_{o}:[\bar{\alpha}] \stackrel{\hat{N}}{\longrightarrow}\left[\bar{\rho}_{1}\right] \stackrel{\widehat{N-2}}{\longrightarrow}\left[\bar{\rho}_{3}\right] \longrightarrow \cdots \longrightarrow\left[\bar{\rho}_{N+m-3}\right] \stackrel{\widehat{2-m}}{\longrightarrow}[\bar{\omega}] \\
& \mathcal{C}_{e}:[\bar{\alpha}] \stackrel{\widehat{1+m}}{\longrightarrow}\left[\bar{\sigma}_{1+m}\right] \stackrel{\widehat{3+m}}{\longrightarrow}\left[\bar{\sigma}_{3+m}\right] \longrightarrow \cdots \longrightarrow\left[\bar{\sigma}_{N-5}\right] \stackrel{\widehat{N-3}}{\longrightarrow}\left[\bar{\sigma}_{N-3}\right] \stackrel{\widehat{N-1}}{\longrightarrow}[\bar{\omega}]
\end{aligned}
$$

where $m=N \bmod 2$. There are resolutions $\tilde{\mathcal{C}}_{o}$ and $\tilde{\mathcal{C}}_{e}$ in $A^{(b)}(N, N-2)$, both starting with $\bar{\alpha}$ and both ending with $\bar{\omega}$,

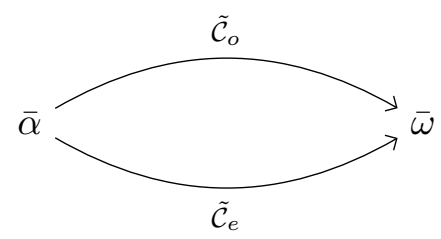

Using the correspondence between elements of $A^{(b)}(N, N-2)$ and maximal chains of $T(N, N-3)$ (see Remark 2.16), each of the two resolutions corresponds to a sequence of maximal chains of $T(N, N-3)$. For $\bar{\rho} \in A^{(b)}(N, N-2)$, let $\mathcal{C}_{\bar{\rho}}$ be the corresponding maximal chain of $T(N, N-3)$. The resolution $\tilde{\mathcal{C}}_{o}$, respectively $\tilde{\mathcal{C}}_{e}$, is then a rule for deforming $\mathcal{C}_{\bar{\alpha}}$ stepwise into $\mathcal{C}_{\bar{\omega}}$.

\footnotetext{
${ }^{14}$ If the sets are supplied with a linear structure, we may as well consider tensor products or direct sums.
} 
The resolution of $T(N, N-2)$, given by $\tilde{\mathcal{C}}_{o}$ and $\tilde{\mathcal{C}}_{e}$, can be regarded as a rule to construct a polyhedron. If $N$ is odd, i.e., $N=2 n+1$, then $\bar{\alpha}$ and $\bar{\omega}$ have both $n(n+1) / 2$ elements. The construction rules are then exactly the same as in the case treated in Section 3.1. Each appearance of an inversion corresponds to a $2 n$-gon.

If $N$ is even, i.e., $N=2 n$, then $\bar{\alpha}$ has $n(n+1) / 2$ elements and $\bar{\omega}$ has $n(n-1) / 2$ elements. Starting from the top vertex, the chain corresponding to $\bar{\alpha}(\bar{\omega})$ is drawn counterclockwise (clockwise). The two chains $\bar{\alpha}$ and $\bar{\omega}$ then join to form an $n^{2}$-gon. Again, the two sides of the $N$-gon equation correspond to sequences of maximal chains that deform $\bar{\alpha}$ into $\bar{\omega}$. But in this case we do not obtain a zonohedron, since an inversion is represented by a $(2 n-1)$-gon, hence an odd polygon.

\subsection{Polygon equations and associated polyhedra}

Let $N \in \mathbb{N}, N>1$, and $0 \leq n \leq N-1$. With $\bar{\rho} \in A^{(b)}(N, n)$ we associate the corresponding Cartesian product $\mathcal{U}_{\bar{\rho}}$ of sets $\mathcal{U}_{J}, J \in \bar{\rho}$. For each $K \in\left(\begin{array}{l}{[N]} \\ n+1\end{array}\right)$, let there be a map

$$
\mathcal{T}_{K}: \mathcal{U}_{\vec{P}_{o}(K)} \rightarrow \mathcal{U}_{\overleftarrow{P_{e}}(K)}
$$

where $\overrightarrow{P_{o}}(K)$ and $\overleftarrow{P_{e}}(K)$ have been defined in Section 2.2 , and $\mathcal{U}_{\vec{P}_{o}}(K), \mathcal{U}_{\overleftarrow{P_{e}}(K)}$ are the corresponding Cartesian products, i.e.,

$$
\begin{aligned}
& \mathcal{U}_{\vec{P}_{o}(K)}:=\mathcal{U}_{K \backslash\left\{k_{n+1}\right\}} \times \mathcal{U}_{K \backslash\left\{k_{n-1}\right\}} \times \cdots \times \mathcal{U}_{K \backslash\left\{k_{1+(n \bmod 2)}\right\}}, \\
& \mathcal{U}_{\overleftarrow{\widehat{P}_{e}}(K)}:=\mathcal{U}_{K \backslash\left\{k_{2-(n \bmod 2)}\right\}} \times \cdots \times \mathcal{U}_{K \backslash\left\{k_{n-2}\right\}} \times \mathcal{U}_{K \backslash\left\{k_{n}\right\}}
\end{aligned}
$$

Remark 4.1. In case of the dual $B^{(r)}(N, n)$ of the Tamari order $T(N, n)$, we are dealing instead with maps $\mathcal{S}_{K}: \mathcal{U}_{\vec{P}_{e}(K)} \longrightarrow \mathcal{U}_{\overleftarrow{P}_{o}(K)}$

Let $[\bar{\rho}] \stackrel{K}{\longrightarrow}\left[\bar{\rho}^{\prime}\right], K \in\left(\begin{array}{c}{[N]} \\ n+1\end{array}\right)$, be an inversion in $T(N, n)$. Hence $\overrightarrow{P_{o}}(K) \subset \bar{\rho}$ and $\overleftarrow{P_{e}}(K) \subset \bar{\rho}^{\prime}$ (where $\subset$ means subsequence). If $\vec{P}_{o}(K)$ appears in $\bar{\rho}$ at consecutive positions, starting at position $\boldsymbol{a}$, we extend $\mathcal{T}_{K}$ to a map $\mathcal{T}_{K, \boldsymbol{a}}: \mathcal{U}_{\bar{\rho}} \rightarrow \mathcal{U}_{\bar{\rho}^{\prime}}$, which acts non-trivially only on the sets labeled by the elements of $P_{o}(K)$.

For a maximal chain $\mathcal{C}:[\bar{\alpha}] \stackrel{K_{1}}{\longrightarrow}\left[\bar{\rho}_{1}\right] \stackrel{K_{2}}{\longrightarrow}\left[\bar{\rho}_{2}\right] \longrightarrow \cdots \stackrel{K_{r}}{\longrightarrow}[\bar{\omega}]$ of $T(N, n)$, let $\tilde{\mathcal{C}}$ be a resolution of $\mathcal{C}$ in $A^{(b)}(N, n)$. We write $\mathcal{T}_{\tilde{\mathcal{C}}}: \mathcal{U}_{\bar{\alpha}} \rightarrow \mathcal{U}_{\bar{\omega}}$ for the corresponding composition of maps $\mathcal{T}_{K_{i}, a_{i}}$ and $\mathcal{P}_{\boldsymbol{a}} \cdot{ }^{15}$ Turning to $T(N, N-2)$ and choosing $\alpha$ as the lexicographically ordered set $\left(\begin{array}{c}{[N]} \\ N-2\end{array}\right)$ and $\omega$ as $\alpha$ in reverse order, the $N$-gon equation is defined by

$$
\mathcal{T}_{\tilde{\mathcal{C}}_{o}}=\mathcal{T}_{\tilde{\mathcal{C}}_{e}}
$$

which is independent of the choice of resolutions.

For odd $N$, i.e., $N=2 n+1, \bar{\alpha}$ is the lexicographically ordered sequence of elements $(2 j \widehat{(2 k}+1)$, with $j=1, \ldots, n$ and $k=j, \ldots, n$. $\bar{\omega}$ is the reverse lexicographically ordered sequence of elements $\left(2 j \widehat{-1)}(2 k)\right.$, where $j=1, \ldots, n$ and $k=j, \ldots, n$. Here $\mathcal{T}_{\hat{k}}: \mathcal{U}_{\overrightarrow{P_{o}}(\hat{k})} \rightarrow \mathcal{U}_{\overleftarrow{P_{e}}(\hat{k})}$ acts between Cartesian products having $n$ factors. In terms of ${ }^{16}$

$$
\hat{\mathcal{T}}_{\hat{k}}=\mathcal{T}_{\hat{k}} \mathcal{P}_{\hat{k}}: \mathcal{U}_{\overleftarrow{P_{o}}(\hat{k})} \rightarrow \mathcal{U}_{\overleftarrow{P_{e}}(\hat{k})}
$$

\footnotetext{
${ }^{15} \mathcal{T}_{\tilde{\mathcal{C}}}$ has the form $\mathcal{P}_{\bar{\omega}, \bar{\rho}_{r}^{\prime}} \mathcal{T}_{K_{r}, \boldsymbol{a}_{r}} \mathcal{P}_{\bar{\rho}_{r-1}, \bar{\rho}_{r-1}^{\prime}} \cdots \mathcal{P}_{\bar{\rho}_{2}, \bar{\rho}_{2}^{\prime}} \mathcal{T}_{K_{2}, \boldsymbol{a}_{2}} \mathcal{P}_{\bar{\rho}_{1}, \bar{\rho}_{1}^{\prime}} \mathcal{T}_{K_{1}, \boldsymbol{a}_{1}} \mathcal{P}_{\bar{\rho}_{0}, \bar{\alpha}}$. Here, for $i=1, \ldots, r, \bar{\rho}_{i-1} \in\left[\bar{\rho}_{i-1}\right]$ (where $\bar{\rho}_{0} \in[\bar{\alpha}]$ ) such that $\vec{P}_{o}\left(K_{i}\right)$ appears in it at consecutive positions, starting at $\boldsymbol{a}_{i} . \bar{\rho}_{i}^{\prime} \in\left[\bar{\rho}_{i}\right]$ is the result of applying $\mathcal{T}_{K_{i}, a_{i}}$ to $\bar{\rho}_{i}$ (so that $\bar{\rho}_{i}^{\prime}$ contains $\overleftarrow{P_{e}}\left(K_{i}\right)$ at consecutive positions)

${ }^{16}$ The permutation map $\mathcal{P}_{\hat{k}}$ achieves a reversion.
} 
the two sides of the $(2 n+1)$-gon equation become maps $\mathcal{U}_{\text {rev }(\bar{\alpha})} \rightarrow \mathcal{U}_{\bar{\omega}}$, where $\operatorname{rev}(\bar{\alpha})$ is $\bar{\alpha}$ reverse lexicographically ordered. $\bar{\alpha}$ and $\bar{\omega}$ have both $c(n+1,2)=\frac{1}{2} n(n+1)$ elements. The "hatted polygon equation" can be obtained either by substituting $\mathcal{T}_{\hat{k}}=\hat{\mathcal{T}}_{\hat{k}} \mathcal{P}_{\hat{k}}$ in the original polygon equation, or by starting with $\mathcal{U}_{\operatorname{rev}(\bar{\alpha})}$ and stepwise mapping it to $\mathcal{U}_{\bar{\omega}}$, following $\mathcal{C}_{o}$ and $\mathcal{C}_{e}$. It has the form

$$
\hat{\mathcal{T}}_{\hat{1}, \boldsymbol{B}_{1}} \hat{\mathcal{T}}_{\hat{3}, \boldsymbol{B}_{3}} \cdots \hat{\mathcal{T}}_{\widehat{2 n+1}, \boldsymbol{B}_{2 n+1}}=\hat{\mathcal{T}}_{\widehat{2 n}, \boldsymbol{B}_{2 n}} \cdots \hat{\mathcal{T}}_{\hat{4}, \boldsymbol{B}_{4}} \hat{\mathcal{T}}_{\hat{2}, \boldsymbol{B}_{2}}
$$

where $\boldsymbol{B}_{k}=\left(\boldsymbol{b}_{k, 1}, \ldots, \boldsymbol{b}_{k, n}\right)$, with $1 \leq \boldsymbol{b}_{k, i} \leq c(n+1,2)$, is the multi-index (increasing sequence of positive integers) specifying the positions, in the respectice active linear order, which take part in the action of the map $\hat{\mathcal{T}}_{\hat{k}}$. Examples will be presented below.

For even $N$, i.e., $N=2 n, \bar{\alpha}$ is the lexicographically ordered sequence $(2 j \widehat{-1)}(2 k)$, where $j=$ $1, \ldots, n$ and $k=j, \ldots, n$, and $\bar{\omega}$ is the reverse lexicographically ordered sequence $(2 j \widehat{(2 k}+1)$, $j=1, \ldots, n-1$ and $k=j, \ldots, n-1$. Thus, $\bar{\alpha}$ has $c(n+1,2)$ and $\bar{\omega}$ has $c(n, 2)$ elements. Now $\mathcal{T}_{\hat{k}}$ maps $\mathcal{U}_{\vec{P}_{o}(\hat{k})}$, which has $n$ factors, to $\mathcal{U}_{\overleftarrow{P_{e}}(\hat{k})}$, which has $n-1$ factors. Also in this case the polygon equation can be expressed in compact form without the need of permutation maps. But in order to achieve this, we have to modify the range to $\mathcal{U}_{\widehat{0 k}} \times \mathcal{U}_{\overleftarrow{P_{e}}(\hat{k})}$, where the sets $\mathcal{U}_{\widehat{0 k}}$ play the role of placeholders, they are irrelevant for the process of evaluation of the polygon equation. We define

$$
\hat{\mathcal{T}}_{\hat{k}}:=\left(u_{\widehat{0 k}}, \mathcal{T}_{\hat{k}} \mathcal{P}_{\hat{k}}\right): \mathcal{U}_{\overleftarrow{P_{o}}(\hat{k})} \rightarrow \mathcal{U}_{\widehat{0 k}} \times \mathcal{U}_{\overleftarrow{P_{e}}(\hat{k})},
$$

choosing fixed elements $u_{\widehat{0 k}} \in \mathcal{U}_{\widehat{0 k}}$. The $2 n$-gon equation then takes the form

$$
\hat{\mathcal{T}}_{\hat{2}, \boldsymbol{B}_{2}} \hat{\mathcal{T}}_{\hat{4}, \boldsymbol{B}_{4}} \cdots \hat{\mathcal{T}}_{\widehat{2 n}, \boldsymbol{B}_{2 n}}=\hat{\mathcal{T}}_{\widehat{2 n-1}, \boldsymbol{B}_{2 n-1}} \cdots \hat{\mathcal{T}}_{\hat{3}, \boldsymbol{B}_{3}} \hat{\mathcal{T}}_{\hat{1}, \boldsymbol{B}_{1}}
$$

where $\boldsymbol{B}_{k}=\left(\boldsymbol{b}_{k, 1}, \ldots, \boldsymbol{b}_{k, n}\right), 1 \leq \boldsymbol{b}_{k, i} \leq c(n+1,2)$. This requires setting $\mathcal{U}_{\widehat{0(2 l)}}=\mathcal{U}_{0} \widehat{(2 l-1)}$ for $l=1, \ldots, n$. Then both sides of (4.1) are maps $\mathcal{U}_{\operatorname{rev}(\bar{\alpha})} \rightarrow \mathcal{U}_{\widehat{01}} \times \cdots \times \mathcal{U}_{0 \widehat{(2 l-1)}} \times \mathcal{U}_{\bar{\omega}}=$ $\mathcal{U}_{\widehat{02}} \times \cdots \times \mathcal{U}_{\widehat{0(2 l)}} \times \mathcal{U}_{\bar{\omega}}$.

Digon equation. The two maximal chains of $T(2,0)$ lead to the digon equation $\mathcal{T}_{1}=\mathcal{T}_{2}$ for the two maps $\mathcal{T}_{i}: \mathcal{U}_{\varnothing} \rightarrow \mathcal{U}_{\varnothing}$.

Trigon equation. The two maximal chains of $T(3,1)$ are $1 \stackrel{12}{\rightarrow} 2 \stackrel{23}{\rightarrow} 3$ and $1 \stackrel{13}{\rightarrow} 3$. The maps $\mathcal{T}_{i j}: \mathcal{U}_{i} \rightarrow \mathcal{U}_{j}, i<j$, have to satisfy the trigon equation $\mathcal{T}_{23} \mathcal{T}_{12}=\mathcal{T}_{13}$.

Tetragon equation. The two maximal chains of $T(4,2)$ are

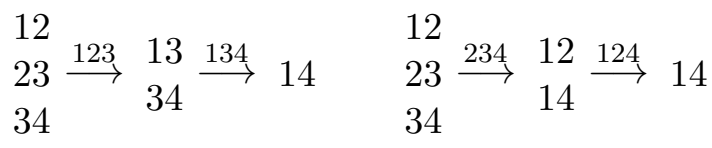

The tetragon equation is thus

$$
\mathcal{T}_{134} \mathcal{T}_{123,1}=\mathcal{T}_{124} \mathcal{T}_{234,2}
$$

for maps $\mathcal{T}_{i j k}: \mathcal{U}_{i j} \times \mathcal{U}_{j k} \rightarrow \mathcal{U}_{i k}, i<j<k$. Using complementary notation, the tetragon equation reads

$$
\mathcal{T}_{\hat{2}} \mathcal{T}_{\hat{4}, \mathbf{1}}=\mathcal{T}_{\hat{3}} \mathcal{T}_{\hat{1}, \mathbf{2}} .
$$




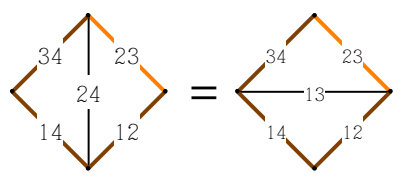

Figure 14. Graphical representation of the tetragon equation (using complementary notation for the edge labels, with hats omitted).

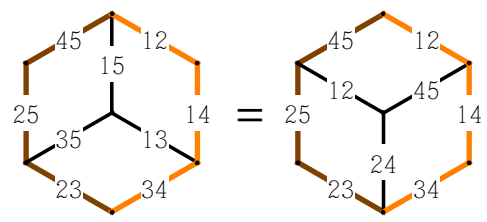

Figure 15. Two complementary sides of the cube formed by $T(5,2)$. We use complementary notation for the labels. The equality presents the pentagon equation in shorthand form. See Fig. 2 for the expanded form (using original labeling).

Also see Fig. 14. The hatted version of the tetragon equation is

$$
\hat{\mathcal{T}}_{\hat{2}, \mathbf{1 3}} \hat{\mathcal{T}}_{\hat{4}, \mathbf{2 3}}=\hat{\mathcal{T}}_{\hat{3}, \mathbf{2 3}} \hat{\mathcal{T}}_{\hat{1}, \mathbf{1 2}},
$$

which can be read off from

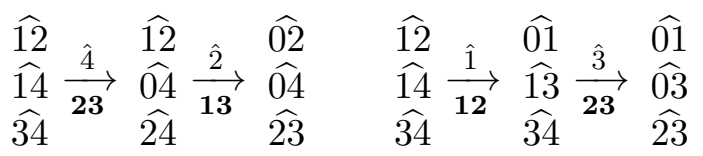

As here, also in the following we will sometimes superfluously display read-off position indices under the arrows.

\begin{tabular}{|c|c|c|c|c|c|c|c|}
\hline & 234 & 234 & 345 & 123 & 123 & 345 & 345 \\
\hline$\longrightarrow$ & $124 \stackrel{1245}{\longrightarrow}$ & $245 \stackrel{2345}{\longrightarrow}$ & 235 & $134 \stackrel{1345}{\longrightarrow}$ & $345 \stackrel{\sim}{\longrightarrow}$ & $123 \stackrel{1235}{\longrightarrow}$ & 235 \\
\hline & 145 & 125 & 125 & 145 & 135 & 135 & \\
\hline
\end{tabular}

Pentagon equation. The two maximal chains of $T(5,3)$ can be resolved to

They describe deformations of maximal chains of $T(5,2)$, see Fig. 2. Here we consider maps $\mathcal{T}_{i j k l}: \mathcal{U}_{i j k} \times \mathcal{U}_{i k l} \rightarrow \mathcal{U}_{j k l} \times \mathcal{U}_{i j l}, i<j<k<l$. Using complementary notation, the pentagon equation is thus

$$
\mathcal{T}_{\hat{1}, \mathbf{1}} \mathcal{T}_{\hat{3}, \mathbf{2}} \mathcal{T}_{\hat{5}, \mathbf{1}}=\mathcal{T}_{\hat{4}, \mathbf{2}} \mathcal{P}_{\mathbf{1}} \mathcal{T}_{\hat{2}, \mathbf{2}}
$$

also see Fig. 15. In terms of $\hat{\mathcal{T}}:=\mathcal{T} \mathcal{P}$, it takes the form

$$
\hat{\mathcal{T}}_{\hat{1}, \mathbf{1 2}} \hat{\mathcal{T}}_{\hat{3}, \mathbf{1 3}} \hat{\mathcal{T}}_{\hat{5}, \mathbf{2 3}}=\hat{\mathcal{T}}_{\hat{4}, \mathbf{2 3}} \hat{\mathcal{T}}_{\hat{2}, \mathbf{1 2}} .
$$

Hexagon equation. We will treat this case in some more detail. The two maximal chains of $T(6,4)$ are

$$
\mathcal{C}_{o}:[\bar{\alpha}] \stackrel{12345}{\longrightarrow}\left[\bar{\rho}_{1}\right] \stackrel{12356}{\longrightarrow}\left[\bar{\rho}_{3}\right] \stackrel{13456}{\longrightarrow}[\bar{\omega}], \quad \mathcal{C}_{e}:[\bar{\alpha}] \stackrel{23456}{\longrightarrow}\left[\bar{\sigma}_{1}\right] \stackrel{12456}{\longrightarrow}\left[\bar{\sigma}_{3}\right] \stackrel{12346}{\longrightarrow}[\bar{\omega}],
$$




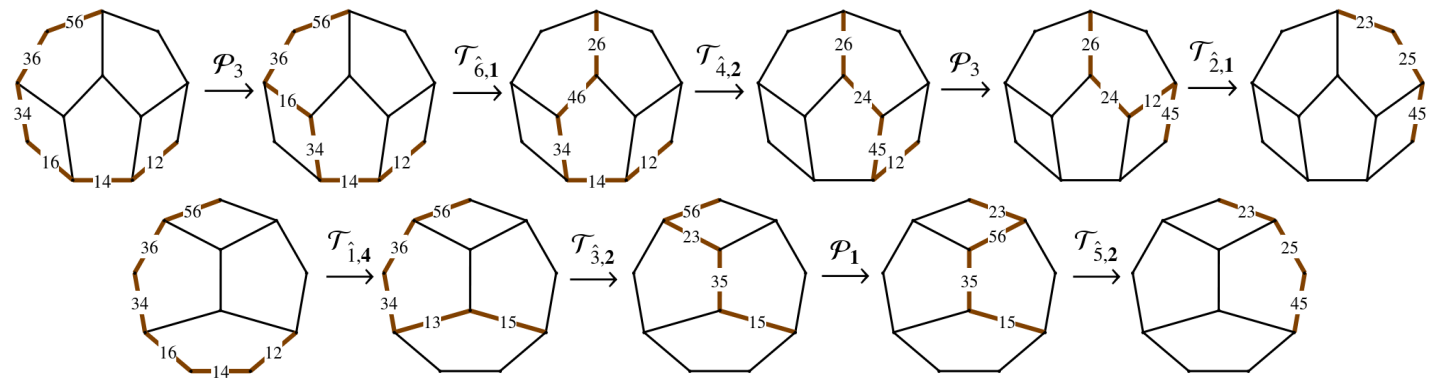

Figure 16. The left-hand side of the hexagon equation (4.4) corresponds to a sequence of maximal chains (first row) on one side of the associahedron in three dimensions, formed by the Tamari lattice $T(6,3)$. The right-hand side corresponds to a sequence of maximal chains (second row) on the complementary side.

with $\bar{\alpha}=(1234,1245,1256,2345,2356,3456)$. The equivalence class $[\bar{\alpha}]$ contains another linear order, which is $(1234,1245,2345,1256,2356,3456)$. We obtain

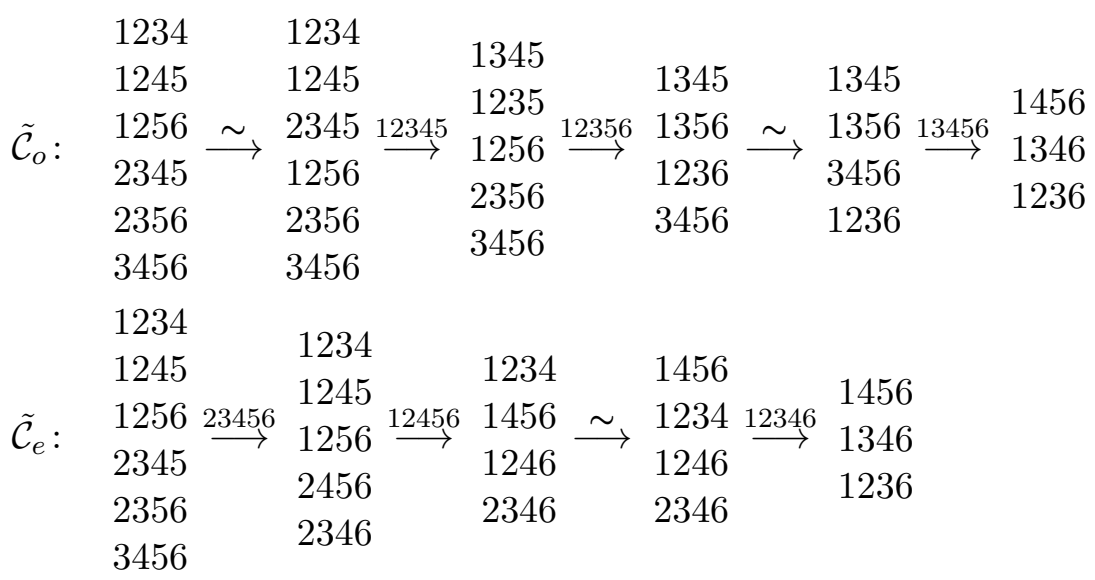

In this case, we consider maps $\mathcal{T}_{i j k l m}: \mathcal{U}_{i j k l} \times \mathcal{U}_{i j l m} \times \mathcal{U}_{j k l m} \rightarrow \mathcal{U}_{i k l m} \times \mathcal{U}_{i j k m}, i<j<k<l<m$. Using complementary notation, the chains $\tilde{\mathcal{C}}_{o}$ and $\tilde{\mathcal{C}}_{e} \operatorname{read}^{17}$

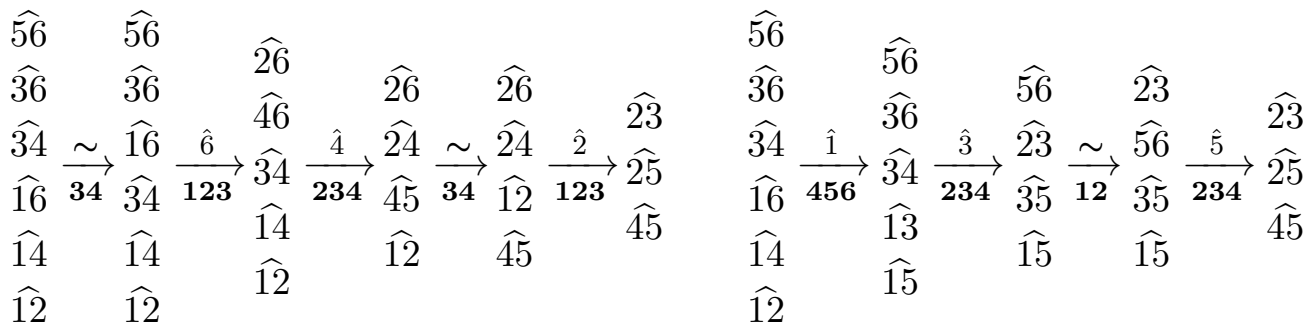

This corresponds to the two sequences of graphs in Fig. 16. We read off the hexagon equation

$$
\mathcal{T}_{\hat{\mathrm{Q}}, \mathbf{1}} \mathcal{P}_{\mathbf{3}} \mathcal{T}_{\hat{4}, \mathbf{2}} \mathcal{T}_{\hat{\mathrm{b}}, \mathbf{1}} \mathcal{P}_{\mathbf{3}}=\mathcal{T}_{\hat{\mathrm{S}}, \mathbf{2}} \mathcal{P}_{\mathbf{1}} \mathcal{T}_{\hat{3}, \mathbf{2}} \mathcal{T}_{\hat{1}, \mathbf{4}}
$$

Fig. 17 is a short-hand form of Fig. 16. In a categorical setting, a similar diagram appeared in [50, p. 218], and in [94, p. 189] as a 4-cycle condition. According to the prescription given for even polygon equations in the beginning of this subsection, we obtain the following hatted version of the hexagon equation,

$$
\hat{\mathcal{T}}_{\hat{2}, \mathbf{1 4 5}} \hat{\mathcal{T}}_{\hat{\mathrm{T}} \mathbf{2 4 6}} \hat{\mathcal{T}}_{\hat{\hat{6}}, \mathbf{3 5 6}}=\hat{\mathcal{T}}_{\hat{\mathrm{S}}, \mathbf{3 5 6}} \hat{\mathcal{T}}_{\hat{3}, \mathbf{2 4 5}} \hat{\mathcal{T}}_{\hat{1}, \mathbf{1 2 3}},
$$

\footnotetext{
${ }^{17}$ Again, the boldface digits are positions, counted from top to bottom in a column, on which the corresponding action takes place. Here they are always consecutive and can thus be abbreviated to the first, as done in (4.4).
} 


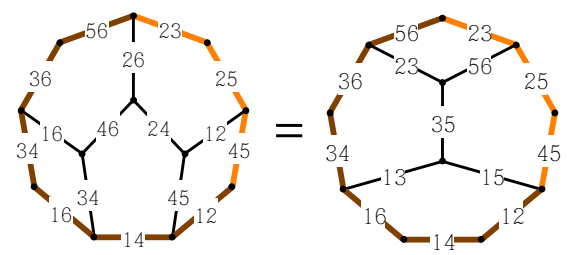

Figure 17. Two complementary sides of the associahedron. The equality expresses the hexagon equation.

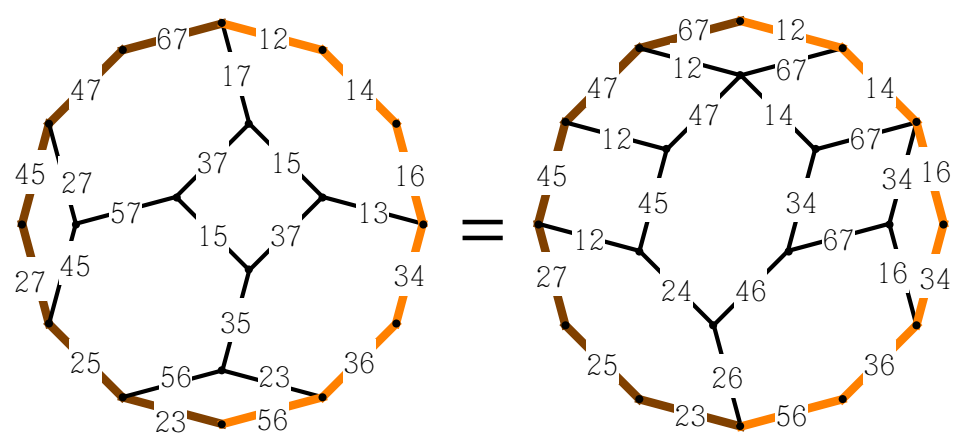

Figure 18. Two complementary sides of the Edelman-Reiner polyhedron, formed by $T(7,4)$. The equality represents the heptagon equation.

which can be read off from

\begin{tabular}{|c|c|c|c|c|c|c|c|c|c|}
\hline$\widehat{12}$ & $\widehat{12}$ & $\widehat{12}$ & $\widehat{02}$ & $\widehat{12}$ & & $\widehat{01}$ & & $\widehat{01}$ & \\
\hline$\widehat{14}$ & $\widehat{14}$ & $\widehat{04}$ & $\widehat{04}$ & $\widehat{14}$ & & $\widehat{13}$ & & $\widehat{03}$ & \\
\hline$\widehat{16}$ & $\widehat{06}$ & $\widehat{06}$ & $\widehat{06}$ & $\widehat{16}$ & $\hat{1}$ & $\widehat{15}$ & $\hat{3}$ & $\widehat{15}$ & $\hat{5}$ \\
\hline 356 & $\widehat{34} \overrightarrow{246}$ & $\overrightarrow{145}$ & $\widehat{23}$ & $\widehat{34}$ & $\overrightarrow{123}$ & $\widehat{34}$ & $\overrightarrow{245}$ & $\widehat{23}$ & $\overrightarrow{356}$ \\
\hline 3 & $\widehat{26}$ & $\widehat{26}$ & $\widehat{25}$ & $\widehat{36}$ & & $\widehat{36}$ & & $\widehat{35}$ & \\
\hline$\widehat{56}$ & $\widehat{46}$ & $\widehat{45}$ & $\widehat{45}$ & $\widehat{56}$ & & $\widehat{56}$ & & $\widehat{56}$ & \\
\hline
\end{tabular}

Some versions of (4.5) appeared in [54, 61, 62, 63, 64, 65, 66] as a "Pachner relation" for a map realizing Pachner moves of triangulations of a four-dimensional manifold.

Heptagon equation. Here we consider maps $\mathcal{T}_{i j k l m p}: \mathcal{U}_{i j k l m} \times \mathcal{U}_{i j k m p} \times \mathcal{U}_{i k l m p} \rightarrow \mathcal{U}_{j k l m p} \times$ $\mathcal{U}_{i j l m p} \times \mathcal{U}_{i j k l p}, i<j<k<l<m<p$. The two maximal chains of $T(7,5)$ lead to the heptagon equation

$$
\mathcal{T}_{\hat{1}, \mathbf{1}} \mathcal{T}_{\hat{3}, \mathbf{3}} \mathcal{P}_{\mathbf{5}} \mathcal{P}_{\mathbf{2}} \mathcal{T}_{\hat{\mathrm{S}}, \mathbf{3}} \mathcal{T}_{\hat{\mathrm{\gamma}}, \mathbf{1}} \mathcal{P}_{\mathbf{3}}=\mathcal{P}_{\mathbf{3}} \mathcal{T}_{\hat{6}, \mathbf{4}} \mathcal{P}_{\mathbf{3}} \mathcal{P}_{\mathbf{2}} \mathcal{P}_{\mathbf{1}} \mathcal{T}_{\hat{4}, \mathbf{3}} \mathcal{P}_{\mathbf{2}} \mathcal{P}_{\mathbf{3}} \mathcal{T}_{\hat{2}, \mathbf{4}}
$$

using complementary notation. Fig. 18 shows the two sides of the Edelman-Reiner polyhedron [30], formed by $T(7,4)$, on which the respective sides of this equation correspond to sequences of maximal chains. In terms of $\hat{\mathcal{T}}:=\mathcal{T} \mathcal{P}_{\mathbf{1 3}}$, the heptagon equation takes the form

$$
\hat{\mathcal{T}}_{\hat{1}, \mathbf{1 2 3}} \hat{\mathcal{T}}_{\hat{3}, \mathbf{1 4 5}} \hat{\mathcal{T}}_{\hat{5}, \mathbf{2 4 6}} \hat{\mathcal{T}}_{\hat{\bar{T}}, \mathbf{3 5 6}}=\hat{\mathcal{T}}_{\hat{6}, \mathbf{3 5 6}} \hat{\mathcal{T}}_{\hat{4}, \mathbf{2 4 5}} \hat{\mathcal{T}}_{\hat{2}, \mathbf{1 2 3}} \text {. }
$$

An equation with this structure appeared in [100].

Octagon equation. In case of $T(8,6)$, we consider maps $\mathcal{T}_{i j k l m p q}: \mathcal{U}_{i j k l m p} \times \mathcal{U}_{i j k l p q} \times \mathcal{U}_{i j l m p q} \times$ $\mathcal{U}_{j k l m p q} \rightarrow \mathcal{U}_{i k l m p q} \times \mathcal{U}_{i j k m p q} \times \mathcal{U}_{i j k l m p}, i<j<k<l<m<p<q$, subject to the octagon equation

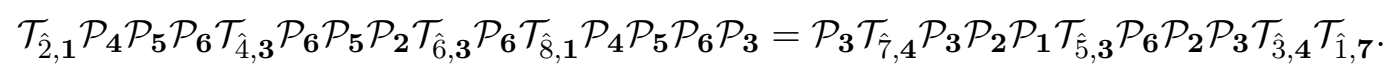




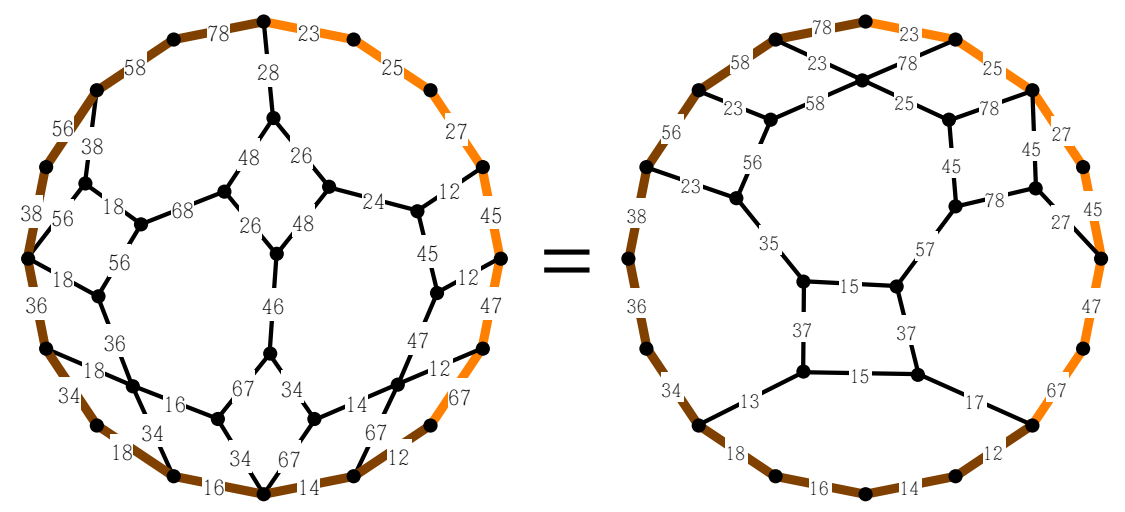

Figure 19. Two complementary sides of the polyhedron formed by $T(8,5)$. Equality represents the octagon equation.

See Fig. 19. The hatted version of the octagon equation is

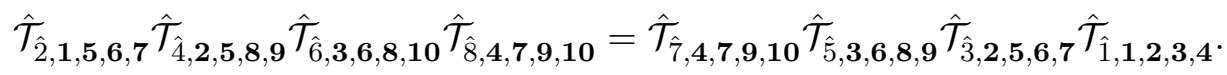

The position indices can be read off from

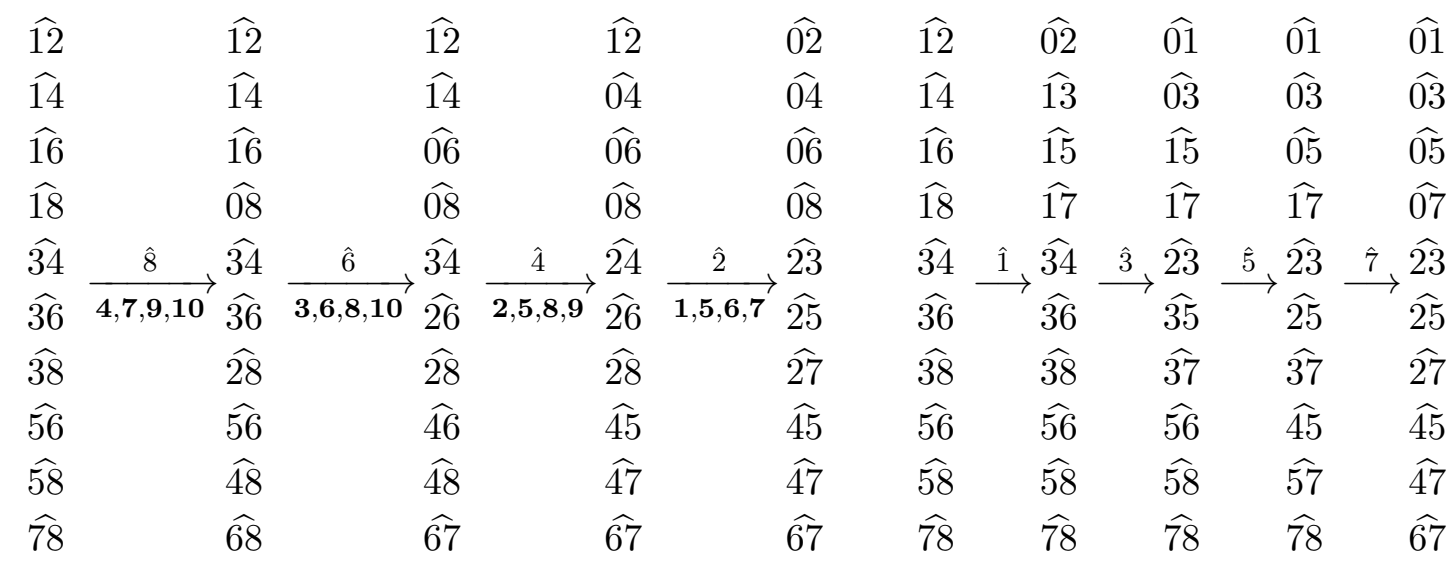

Enneagon equation. For $T(9,7)$ we find

$$
\begin{aligned}
& \mathcal{T}_{\hat{1}, \mathbf{1}} \mathcal{T}_{\hat{3}, 4} \mathcal{P}_{\mathbf{7}} \mathcal{P}_{\mathbf{8}} \mathcal{P}_{\mathbf{9}} \mathcal{P}_{\mathbf{3}} \mathcal{P}_{\mathbf{2}} \mathcal{P}_{\mathbf{4}} \mathcal{T}_{\hat{5}, \mathbf{5}} \mathcal{P}_{\mathbf{8}} \mathcal{P}_{\mathbf{7}} \mathcal{P}_{\mathbf{4}} \mathcal{P}_{\mathbf{3}} \mathcal{T}_{\hat{7}, 4} \mathcal{P}_{\mathbf{7}} \mathcal{T}_{\hat{\mathrm{g}}, \mathbf{1}} \mathcal{P}_{\mathbf{4}} \mathcal{P}_{\mathbf{5}} \mathcal{P}_{\mathbf{6}} \mathcal{P}_{\mathbf{3}} \\
& =\mathcal{P}_{7} \mathcal{P}_{4} \mathcal{P}_{5} \mathcal{P}_{6} \mathcal{P}_{3} \mathcal{T}_{\hat{8}, 7} \mathcal{P}_{6} \mathcal{P}_{5} \mathcal{P}_{4} \mathcal{P}_{3} \mathcal{P}_{2} \mathcal{P}_{1} \mathcal{T}_{\hat{6}, 5} \mathcal{P}_{8} \mathcal{P}_{4} \mathcal{P}_{3} \mathcal{P}_{2} \mathcal{P}_{5} \mathcal{P}_{4} \mathcal{P}_{3} \mathcal{T}_{\hat{4}, 5} \mathcal{P}_{4} \mathcal{P}_{5} \mathcal{P}_{6} \mathcal{T}_{\hat{2}, 7},
\end{aligned}
$$

which can be visualized on $T(9,6)$, see Fig. 20. In terms of $\hat{\mathcal{T}}:=\mathcal{T P}_{\mathbf{1 4}} \mathcal{P}_{\mathbf{2 3}}$, the enneagon (or nonagon) equation takes the compact form

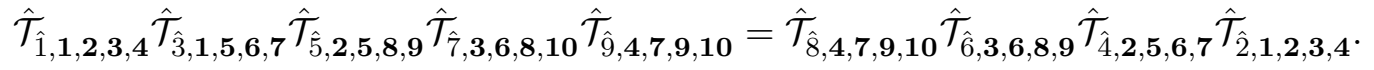

Decagon equation. For $T(10,8)$ we obtain the equation

$$
\begin{aligned}
& \mathcal{T}_{\hat{2}, 1} \mathcal{P}_{5} \mathcal{P}_{6} \mathcal{P}_{7} \mathcal{P}_{8} \mathcal{P}_{\mathbf{9}} \mathcal{P}_{10} \mathcal{T}_{\hat{4}, 4} \mathcal{P}_{8} \mathcal{P}_{\mathbf{9}} \mathcal{P}_{10} \mathcal{P}_{\mathbf{7}} \mathcal{P}_{\mathbf{8}} \mathcal{P}_{\mathbf{9}} \mathcal{P}_{\mathbf{3}} \mathcal{P}_{\mathbf{2}} \mathcal{P}_{4} \mathcal{T}_{\hat{6}, 5} \mathcal{P}_{\mathbf{9}} \mathcal{P}_{10} \mathcal{P}_{\mathbf{8}} \mathcal{P}_{\mathbf{7}} \\
& \mathcal{P}_{4} \mathcal{P}_{3} \mathcal{T}_{\hat{8}, 4} \mathcal{P}_{8} \mathcal{P}_{9} \mathcal{P}_{10} \mathcal{P}_{7} \mathcal{T}_{\widehat{10}, 1} \mathcal{P}_{5} \mathcal{P}_{6} \mathcal{P}_{7} \mathcal{P}_{8} \mathcal{P}_{9} \mathcal{P}_{10} \mathcal{P}_{4} \mathcal{P}_{5} \mathcal{P}_{6} \mathcal{P}_{3} \\
& =\mathcal{P}_{7} \mathcal{P}_{4} \mathcal{P}_{5} \mathcal{P}_{6} \mathcal{P}_{3} \mathcal{T}_{\hat{9}, 7} \mathcal{P}_{6} \mathcal{P}_{5} \mathcal{P}_{4} \mathcal{P}_{3} \mathcal{P}_{2} \mathcal{P}_{1} \mathcal{T}_{\hat{7}, 5} \mathcal{P}_{9} \mathcal{P}_{10} \mathcal{P}_{8} \mathcal{P}_{4} \mathcal{P}_{3} \mathcal{P}_{2} \mathcal{P}_{5} \mathcal{P}_{4} \mathcal{P}_{3} \mathcal{T}_{\hat{5}, 5} \\
& \mathcal{P}_{9} \mathcal{P}_{10} \mathcal{P}_{4} \mathcal{P}_{5} \mathcal{P}_{6} \mathcal{T}_{\hat{3}, 7} \mathcal{T}_{\hat{1}, 11},
\end{aligned}
$$




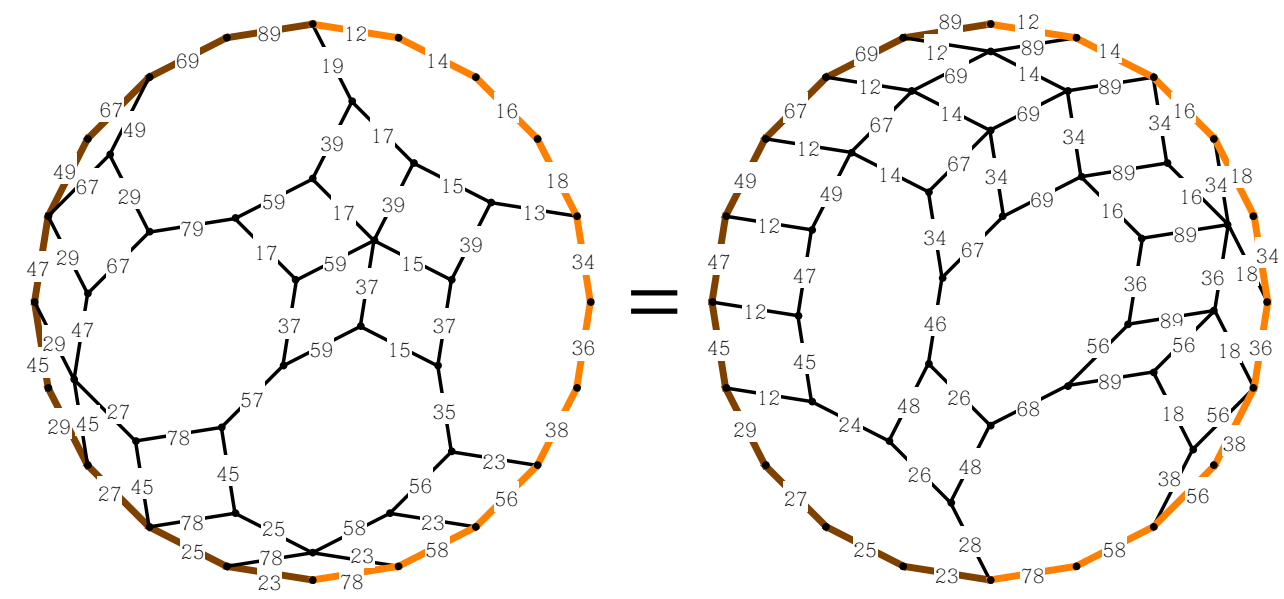

Figure 20. Two complementary sides of the polyhedral part of $T(9,6)$ (which is non-polyhedral due to the existence of small cubes). Equality represents the enneagon equation.
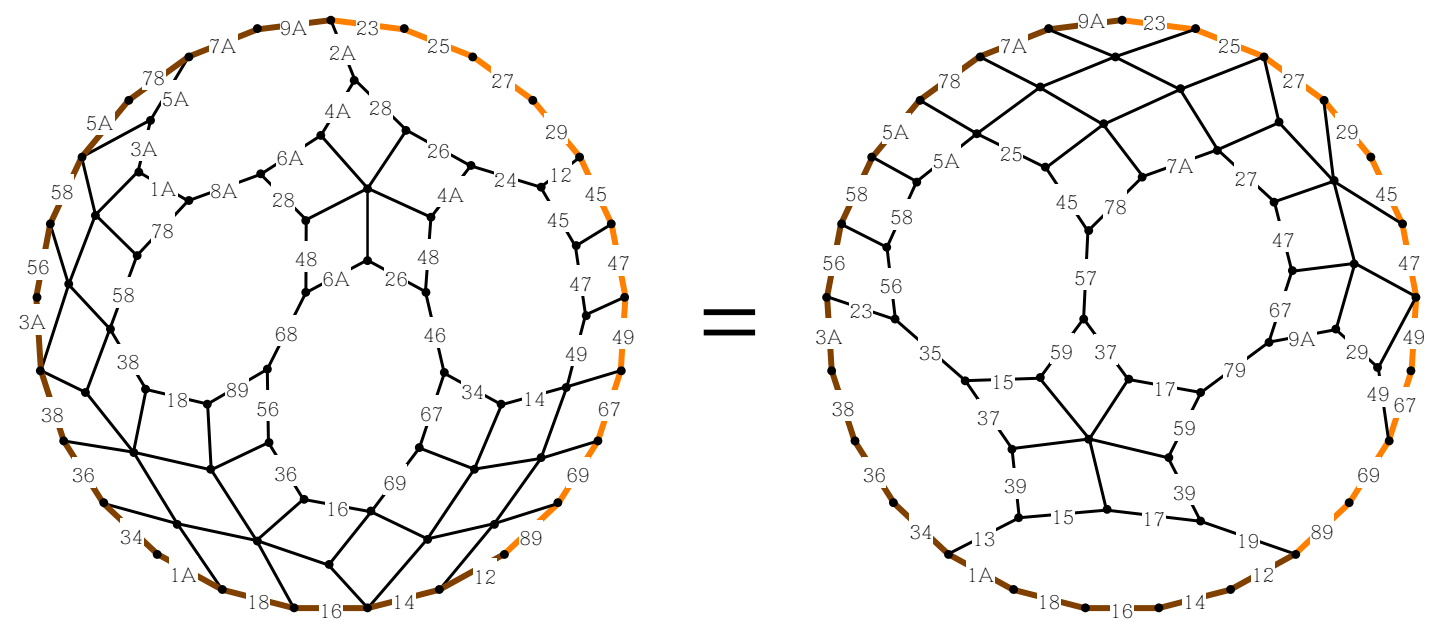

Figure 21. Two complementary sides of the polyhedral part of $T(10,7)$ (small cubes are resolved). Equality represents the decagon equation. Here $A$ stands for 10.

and Fig. 21 shows the corresponding polyhedral representation obtained from $T(10,7)$. The hatted version of the decagon equation is

$$
\begin{aligned}
& \hat{\mathcal{T}}_{\hat{\mathbf{2}}, \mathbf{1}, \mathbf{6}, 7,8,9} \hat{\mathcal{T}}_{\hat{4}, 2,6,10,11,12} \hat{\mathcal{T}}_{\hat{6}, 3,7,10,13,14} \hat{\mathcal{T}}_{\hat{8}, 4,8,11,13,15} \hat{\mathcal{T}}_{\hat{10}, 5,9,12,14,15} \\
& =\hat{\mathcal{T}}_{\hat{9}, 5,9,12,14,15} \hat{\mathcal{T}}_{\hat{\gamma}, 4,8,11,13,14} \hat{\mathcal{T}}_{\hat{\mathrm{S}}, \mathbf{3}, 7,10,11,12} \hat{\mathcal{T}}_{\hat{\mathrm{J}}, 2,6,7,8,9} \hat{\mathcal{T}}_{\hat{1}, 1,2,3,4,5} .
\end{aligned}
$$

Hendecagon equation. For $T(11,9)$, the associated equation reads

$$
\begin{aligned}
\mathcal{T}_{\hat{1}, 1} & \mathcal{T}_{\hat{3}, 5} \mathcal{P}_{9} \mathcal{P}_{10} \mathcal{P}_{11} \mathcal{P}_{12} \mathcal{P}_{13} \mathcal{P}_{14} \mathcal{P}_{4} \mathcal{P}_{3} \mathcal{P}_{2} \mathcal{P}_{5} \mathcal{P}_{4} \mathcal{P}_{6} \mathcal{T}_{\hat{5}, 7} \mathcal{P}_{11} \mathcal{P}_{12} \mathcal{P}_{13} \mathcal{P}_{10} \mathcal{P}_{11} \mathcal{P}_{12} \\
\mathcal{P}_{6} \mathcal{P}_{5} \mathcal{P}_{4} & \mathcal{P}_{3} \mathcal{P}_{7} \mathcal{P}_{6} \mathcal{T}_{\bar{\gamma}, 7} \mathcal{P}_{11} \mathcal{P}_{12} \mathcal{P}_{10} \mathcal{P}_{9} \mathcal{P}_{6} \mathcal{P}_{5} \mathcal{P}_{4} \mathcal{T}_{\hat{9}, 5} \mathcal{P}_{9} \mathcal{P}_{10} \mathcal{P}_{11} \mathcal{P}_{8} \mathcal{T}_{\widehat{11}, 1} \\
\mathcal{P}_{5} \mathcal{P}_{6} \mathcal{P}_{7} \mathcal{P}_{8} \mathcal{P}_{9} \mathcal{P}_{10} \mathcal{P}_{4} \mathcal{P}_{5} \mathcal{P}_{6} \mathcal{P}_{3} & \\
= & \mathcal{P}_{12} \mathcal{P}_{9} \mathcal{P}_{10} \mathcal{P}_{11} \mathcal{P}_{8} \mathcal{P}_{5} \mathcal{P}_{6} \mathcal{P}_{7} \mathcal{P}_{8} \mathcal{P}_{9} \mathcal{P}_{10} \mathcal{P}_{4} \mathcal{P}_{5} \mathcal{P}_{6} \mathcal{P}_{3} \mathcal{T}_{\widehat{10}, 11} \mathcal{P}_{10} \mathcal{P}_{9} \mathcal{P}_{8} \mathcal{P}_{7} \mathcal{P}_{6} \mathcal{P}_{5} \mathcal{P}_{4} \mathcal{P}_{3} \mathcal{P}_{2} \mathcal{P}_{1} \\
& \mathcal{T}_{\hat{8}, 8} \mathcal{P}_{12} \mathcal{P}_{13} \mathcal{P}_{11} \mathcal{P}_{7} \mathcal{P}_{6} \mathcal{P}_{5} \mathcal{P}_{4} \mathcal{P}_{3} \mathcal{P}_{2} \mathcal{P}_{8} \mathcal{P}_{7} \mathcal{P}_{6} \mathcal{P}_{5} \mathcal{P}_{4} \mathcal{P}_{3} \mathcal{T}_{\hat{6}, 7} \mathcal{P}_{11} \mathcal{P}_{12} \mathcal{P}_{6} \mathcal{P}_{5} \mathcal{P}_{4} \mathcal{P}_{7} \mathcal{P}_{6} \mathcal{P}_{5} \\
& \mathcal{P}_{8} \mathcal{P}_{7} \mathcal{P}_{6} \mathcal{T}_{\hat{4}, 8} \mathcal{P}_{7} \mathcal{P}_{8} \mathcal{P}_{9} \mathcal{P}_{10} \mathcal{T}_{\hat{2}, 11},
\end{aligned}
$$



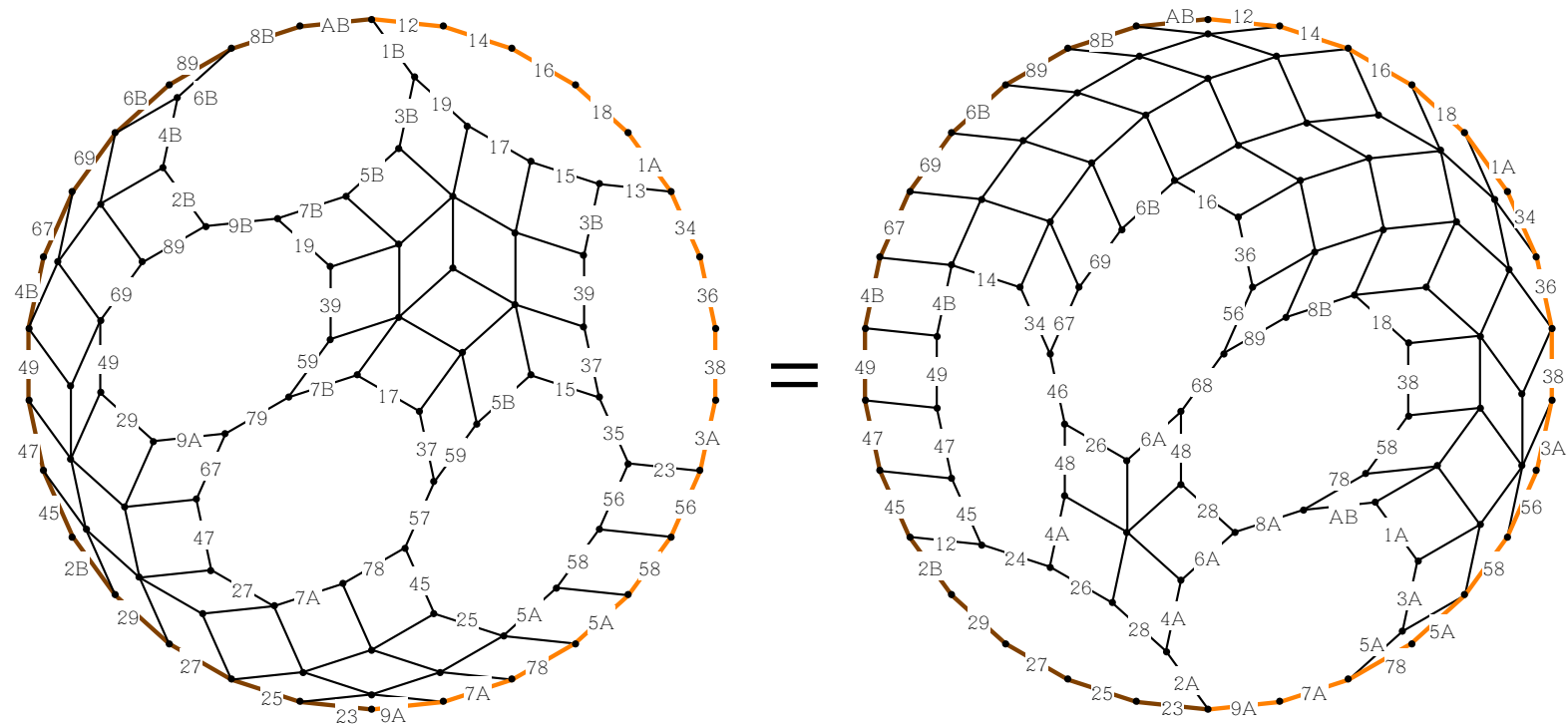

Figure 22. Two complementary sides of the polyhedral part of $T(11,8)$ (small cubes are resolved). Equality represents the hendecagon equation. Here we set $A:=10, B:=11$.

also see Fig. 22. In terms of $\hat{\mathcal{T}}=\mathcal{T} \mathcal{P}_{\mathbf{1 5}} \mathcal{P}_{\mathbf{2 4}}$, it takes the form

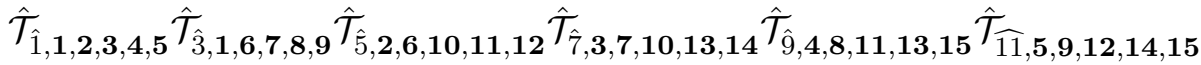

$$
\begin{aligned}
& =\hat{\mathcal{T}}_{\widehat{10}, \mathbf{5}, \mathbf{9}, \mathbf{1 2 , 1 4}, \mathbf{1 5}} \hat{\mathcal{T}}_{\hat{8}, \mathbf{4}, \mathbf{8}, \mathbf{1 1}, \mathbf{1 3 , 1 4}} \hat{\mathcal{T}}_{\hat{6}, \mathbf{3}, \mathbf{7}, \mathbf{1 0}, \mathbf{1 1 , 1 2}} \hat{\mathcal{T}}_{\hat{4}, \mathbf{2}, \mathbf{6}, \mathbf{7}, \mathbf{8}, \mathbf{9}} \hat{\mathcal{T}}_{\hat{\mathrm{2}} \mathbf{1 , 2 , 3 , 4 , 5}} .
\end{aligned}
$$

Remark 4.2. Disregarding the indices that specify on which sets the maps act, the left-hand side of the $N$-simplex equation has the same structure as the left-hand side of the $(2 N+1)$-gon equation (but there is no such relation between the right-hand sides, of course). Accordingly, the corresponding halfs of the associated polyhedra coincide up to the labeling.

\subsection{Lax systems for polygon equations}

In this subsection we consider the case where the maps $\mathcal{T}_{J}, J \in\left(\begin{array}{c}{[N+1]} \\ N-1\end{array}\right)$, are "localized" to maps

$$
\begin{aligned}
\mathcal{L}_{J}: \mathcal{U}_{J} & \longrightarrow \operatorname{Map}\left(\mathcal{U}_{\vec{P}_{o}(J)}, \mathcal{U}_{\overleftarrow{P_{e}}(J)}\right), \\
u_{J} & \longmapsto \mathcal{L}_{J}\left(u_{J}\right): \quad \mathcal{U}_{\vec{P}_{o}(J)} \rightarrow \mathcal{U}_{\overleftarrow{P_{e}}(J)}
\end{aligned}
$$

In $T(N+1, N-2)$, counterparts of the two maximal chains, of which $T(N, N-2)$ consists, appear as chains for all $\hat{k} \in\left(\begin{array}{c}{[N+1]} \\ N\end{array}\right), k \in[N+1]$,

$$
\begin{aligned}
& \mathcal{C}_{\hat{k}, o}:\left[\bar{\alpha}_{\hat{k}}\right] \stackrel{\widehat{k k_{N}}}{\longrightarrow}\left[\bar{\rho}_{\hat{k}, 1}\right] \stackrel{k \widehat{k_{N-2}}}{\longrightarrow}\left[\bar{\rho}_{\hat{k}, 2}\right] \longrightarrow \cdots\left[\bar{\rho}_{\hat{k}, N+m-3}\right] \stackrel{k \widehat{k k_{2-m}}}{\longrightarrow}\left[\bar{\omega}_{\hat{k}}\right], \\
& \mathcal{C}_{\hat{k}, e}:\left[\bar{\alpha}_{\hat{k}}\right] \stackrel{k \widehat{k_{1+m}}}{\longrightarrow}\left[\bar{\sigma}_{\hat{k}, 1}\right] \stackrel{\sqrt{k_{3+m}}}{\longrightarrow}\left[\bar{\sigma}_{\hat{k}, 2}\right] \longrightarrow \cdots \stackrel{\widehat{k \widehat{k_{N-1}}}}{\longrightarrow}\left[\bar{\omega}_{\hat{k}}\right],
\end{aligned}
$$

where we wrote $\hat{k}=\left(k_{1}, \ldots, k_{N}\right), k_{1}<k_{2}<\cdots<k_{N}$. Here $\bar{\alpha}_{\hat{k}}, \bar{\omega}_{\hat{k}}, \bar{\rho}_{\hat{k}, i}$ and $\bar{\sigma}_{\hat{k}, i}$ are reduced admissible linear orders of $\left(\begin{array}{c}\hat{k} \\ N-2\end{array}\right)$, and $m:=N \bmod 2$. Let $\tilde{\mathcal{C}}_{\hat{k}, o}$ and $\tilde{\mathcal{C}}_{\hat{k}, e}$ be resolutions of the above chains. We consider the following system of localized $N$-gon equations,

$$
\mathcal{L}_{\tilde{\mathcal{C}}_{\hat{k}, o}}\left(\boldsymbol{u}_{\vec{P}_{o}(\hat{k})}\right)=\mathcal{L}_{\tilde{\mathcal{C}}_{\hat{k}, e}}\left(\boldsymbol{v}_{\overleftarrow{P}_{e}(\hat{k})}\right), \quad \hat{k} \in\left(\begin{array}{c}
{[N+1]} \\
N
\end{array}\right)
$$


where

$$
\boldsymbol{u}_{\overrightarrow{P_{o}}(\hat{k})}=\left(u_{\widehat{k k_{N}}}, u_{k \widehat{k_{N-2}}}, \ldots, u_{k \widehat{k_{2-m}}}\right), \quad \boldsymbol{v}_{\overleftarrow{P_{e}}(\hat{k})}=\left(v_{k \widehat{k_{1+m}}}, \ldots, v_{k \widehat{k_{N-3}}}, \ldots, v_{k \widehat{k_{N-1}}}\right)
$$

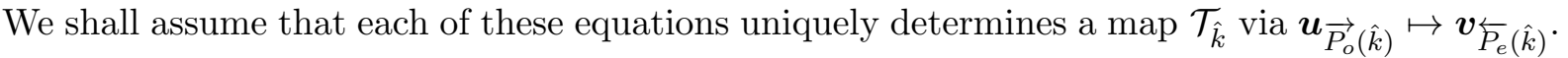

A hatted version of $\mathcal{L}_{J}$ is defined in the same way as the hatted version of $\mathcal{T}_{J}$ (see the beginning of Section 4), differently for even and odd polygon equations. The above system (4.8) then has the form

$$
\begin{aligned}
& \hat{\mathcal{L}}_{k k_{2-m}}, \boldsymbol{Y}_{\boldsymbol{B}_{2-m}} \cdots \hat{\mathcal{L}}_{k \widehat{k_{N-2}}, \boldsymbol{Y}_{\boldsymbol{B}_{N-2}}} \hat{\mathcal{L}}_{\widehat{k k_{N}}, \boldsymbol{Y}_{\boldsymbol{B}_{N}}} \\
& =\left(\hat{\mathcal{L}}_{k k_{N-1}}, \boldsymbol{Y}_{\boldsymbol{B}_{N-1}} \hat{\mathcal{L}}_{k k_{N-3}, \boldsymbol{Y}_{\boldsymbol{B}_{N-3}}} \cdots \hat{\mathcal{L}}_{k k_{m+1}, \boldsymbol{Y}_{\boldsymbol{B}_{m+1}}}\right) \circ \mathcal{T}_{\hat{k}},
\end{aligned}
$$

where $\hat{k} \in\left(\begin{array}{c}{[N+1]} \\ N\end{array}\right)$. Here $\boldsymbol{Y}_{\boldsymbol{B}_{i}}=\left(\boldsymbol{y}_{\boldsymbol{b}_{i, 1}}, \ldots, \boldsymbol{y}_{\boldsymbol{b}_{i, n}}\right)$, where $n=\lfloor N / 2\rfloor=(N-m) / 2$, is an increasing sequence of integers, and $\boldsymbol{B}_{i}=\left(\boldsymbol{b}_{i, 1}, \ldots, \boldsymbol{b}_{i, n}\right)$ is a multi-index, as defined previously. We have $1 \leq \boldsymbol{b}_{i, j} \leq c(n+1,2)$ and $1 \leq \boldsymbol{y}_{\boldsymbol{b}_{i, j}} \leq c(n+m+1,3)$.

With $\bar{\rho}=\left(J_{1}, \ldots, J_{r}\right) \in A^{(b)}(N+1, N-1)$ we associate the composition of maps $\hat{\mathcal{L}}_{\bar{\rho}}=$ $\hat{\mathcal{L}}_{J_{r}, \boldsymbol{B}_{r}} \cdots \hat{\mathcal{L}}_{J_{1}, \boldsymbol{B}_{1}}$. The domain of $\hat{\mathcal{L}}_{\bar{\rho}}$ is $\mathcal{U}_{\text {rev }(\bar{\eta})}$, where $\eta \in A(N+1, N-1)$ is the lexicographical order of $\left(\begin{array}{c}{[N+1]} \\ N-2\end{array}\right), B_{1}$ is the multi-index of the positions of the elements of $P\left(J_{1}\right)$ in $\operatorname{rev}(\bar{\eta})$ (which has $c(n+m+1,3)$ elements). It seems to be a difficult task to find a general formula that determines the other multi-indices.

In a similar way as in the case of simplex equations, one can show that the $(N+1)$-gon equation $\mathcal{T}_{\tilde{\mathcal{C}}_{o}}=\mathcal{T}_{\tilde{\mathcal{C}}_{e}}$, where $\tilde{\mathcal{C}}_{o}$ and $\tilde{\mathcal{C}}_{e}$ constitute a resolution of $T(N+1, N-1)$, arises as a consistency condition of the above Lax system. Here one starts with $\hat{\mathcal{L}}_{\bar{\alpha}}, \bar{\alpha} \in A^{(b)}(N+1, N-1)$, where $\alpha$ is the lexicographical order of $\left(\begin{array}{c}{[N+1]} \\ N-1\end{array}\right)$, and follows the two resolutions.

Example 4.3. For $N=6$, we have

\begin{tabular}{|c|c|c|c|c|c|c|c|c|c|c|c|}
\hline & $\widehat{123}$ & & $\widehat{123}$ & & $\widehat{123}$ & & $\widehat{123}$ & & $\widehat{123}$ & & $\widehat{023}$ \\
\hline & $\widehat{125}$ & & $\widehat{125}$ & & $\widehat{125}$ & & $\widehat{125}$ & & $\widehat{025}$ & & $\widehat{025}$ \\
\hline & 127 & & 127 & & 127 & & 027 & & $\widehat{027}$ & & 027 \\
\hline & $\widehat{145}$ & & 145 & & $\widehat{045}$ & & $\widehat{045}$ & & $\widehat{045}$ & & 45 \\
\hline$\widehat{67}$ & $\widehat{147}$ & $\widehat{47}$ & $\widehat{047}$ & $\widehat{45}$ & $\widehat{047}$ & $\widehat{27}$ & $\widehat{047}$ & $\widehat{25}$ & $\widehat{047}$ & $\widehat{23}$ & $\overline{047}$ \\
\hline 690 & $\widehat{067}$ & 580 & $\widehat{067}$ & 470 & $\widehat{067}$ & 389 & $\widehat{067}$ & 279 & $\widehat{067}$ & 178 & \\
\hline & $\widehat{345}$ & & $\widehat{345}$ & & $\widehat{245}$ & & $\widehat{245}$ & & $\widehat{235}$ & & \\
\hline & $\widehat{347}$ & & $\widehat{247}$ & & $\widehat{247}$ & & $\widehat{237}$ & & $\widehat{237}$ & & \\
\hline & $\widehat{267}$ & & 267 & & $\widehat{267}$ & & $\widehat{257}$ & & $\overline{256}$ & & \\
\hline & $\widehat{467}$ & & $\overline{457}$ & & $\widehat{456}$ & & $\widehat{456}$ & & $\overline{456}$ & & \\
\hline
\end{tabular}

$$
\operatorname{rev}(\bar{\eta})=(\widehat{123}, \widehat{125}, \widehat{127}, \widehat{145}, \widehat{147}, \widehat{167}, \widehat{345}, \widehat{347}, \widehat{367}, \widehat{567})
$$

and $\bar{\alpha}=(\widehat{67}, \widehat{47}, \widehat{45}, \widehat{27}, \widehat{25}, \widehat{23}) \in A^{(b)}(7,5)$, so that

from which we can read off the position (i.e., boldface) indices of $\hat{\mathcal{L}}_{\bar{\alpha}}$. The Lax system reads

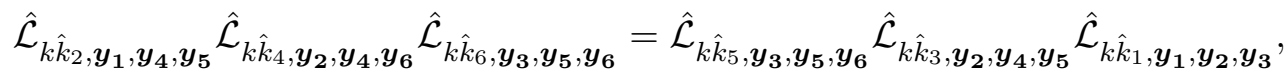

where $1 \leq \boldsymbol{y}_{\boldsymbol{b}} \leq 10$. The consistency condition is now obtained from ${ }^{18}$

$$
\hat{\mathcal{L}}_{\bar{\alpha}}=\hat{\mathcal{L}}_{\widehat{23}, \mathbf{1 7 8}} \hat{\mathcal{L}}_{\widehat{25}, \mathbf{2 7 9}} \hat{\mathcal{L}}_{\widehat{27}, \mathbf{3 8 9}} \hat{\mathcal{L}}_{\widehat{45}, \mathbf{4 7 0}} \hat{\mathcal{L}}_{\widehat{47}, 580} \hat{\mathcal{L}}_{\widehat{67}, 690}
$$

\footnotetext{
${ }^{18}$ Here we depart from our notation and indicate over an equality sign the maps that act on the arguments of the $\hat{\mathcal{L}}$ 's in the respective transformation step. Furthermore, we write $\mathbf{0}$ instead of $\mathbf{1 0}$.
} 


$$
\begin{aligned}
& \stackrel{\mathcal{P}_{\mathbf{3}}}{=} \hat{\mathcal{L}}_{\widehat{23, \mathbf{1 7 8}}} \hat{\mathcal{L}}_{\widehat{25}, \mathbf{2 7 9}} \hat{\mathcal{L}}_{\widehat{45}, \mathbf{4 7 0}} \hat{\mathcal{L}}_{\widehat{27}, \mathbf{3 8 9}} \hat{\mathcal{L}}_{\overparen{47}, \mathbf{5 8 0}} \hat{\mathcal{L}}_{\widehat{67}, \mathbf{6 9 0}} \\
& \stackrel{\mathcal{T}_{\hat{7}, \mathbf{1}}}{=} \hat{\mathcal{L}}_{\widehat{23, \mathbf{1 7 8}}} \hat{\mathcal{L}}_{\widehat{25,279}} \hat{\mathcal{L}}_{\widehat{45}, 470} \hat{\mathcal{L}}_{\overparen{57,690}} \hat{\mathcal{L}}_{\widehat{37,589}} \hat{\mathcal{L}}_{\widehat{17}, \mathbf{3 5 6}} \\
& \stackrel{\mathcal{T}_{\widehat{5}, \mathbf{3}}}{=} \hat{\mathcal{L}}_{\widehat{23,178}} \hat{\mathcal{L}}_{\widehat{56}, \mathbf{6 9 0}} \hat{\mathcal{L}}_{\widehat{35}, 479} \hat{\mathcal{L}}_{\widehat{15}, \mathbf{2 4 6}} \hat{\mathcal{L}}_{\widehat{37}, \mathbf{5 8 9}} \hat{\mathcal{L}}_{\widehat{17}, \mathbf{3 5 6}} \\
& \stackrel{\mathcal{P}_{\mathbf{2}} \mathcal{P}_{\mathbf{5}}}{=} \hat{\mathcal{L}}_{\widehat{56}, \mathbf{6 9 0}} \hat{\mathcal{L}}_{\widehat{23,178}} \hat{\mathcal{L}}_{\widehat{35}, 479} \hat{\mathcal{L}}_{\widehat{37}, 589} \hat{\mathcal{L}}_{\widehat{15}, \mathbf{2 4 6}} \hat{\mathcal{L}}_{\widehat{17}, \mathbf{3 5 6}} \\
& \stackrel{\mathcal{T}_{\hat{3}, \mathbf{3}}}{=} \hat{\mathcal{L}}_{\widehat{56}, \mathbf{6 9 0}} \hat{\mathcal{L}}_{\widehat{36}, \mathbf{5 8 9}} \hat{\mathcal{L}}_{\widehat{34}, \mathbf{4 7 8}} \hat{\mathcal{L}}_{\widehat{13}, \mathbf{1 4 5}} \hat{\mathcal{L}}_{\widehat{15}, \mathbf{2 4 6}} \hat{\mathcal{L}}_{\widehat{17}, \mathbf{3 5 6}} \\
& \stackrel{\mathcal{T}_{\hat{1}, \mathbf{1}}}{=} \hat{\mathcal{L}}_{\widehat{56}, \mathbf{6 9 0}} \hat{\mathcal{L}}_{\widehat{36}, \mathbf{5 8 9}} \hat{\mathcal{L}}_{\widehat{34}, \mathbf{4 7 8}} \hat{\mathcal{L}}_{\widehat{16}, \mathbf{3 5 6}} \hat{\mathcal{L}}_{\widehat{14}, \mathbf{2 4 5}} \hat{\mathcal{L}}_{\widehat{12}, \mathbf{1 2 3}},
\end{aligned}
$$

which is $\hat{\mathcal{L}}_{\bar{\alpha}}=\hat{\mathcal{L}}_{\bar{\omega}} \circ \mathcal{T}_{\tilde{\mathcal{C}}_{o}}$, and

$$
\begin{aligned}
& \hat{\mathcal{L}}_{\bar{\alpha}}=\hat{\mathcal{L}}_{\widehat{23,178}} \hat{\mathcal{L}}_{\widehat{25}, \mathbf{2 7 9} 9} \hat{\mathcal{L}}_{\widehat{27}, \mathbf{3 8 9}} \hat{\mathcal{L}}_{\widehat{45}, \mathbf{4 7 0}} \hat{\mathcal{L}}_{\widehat{47}, \mathbf{5 8 0}} \hat{\mathcal{L}}_{\widehat{67}, \mathbf{6 9 0}} \\
& \stackrel{\mathcal{T}_{\hat{2}, \mathbf{4}}}{=} \hat{\mathcal{L}}_{\widehat{26}, \mathbf{3 8 9}} \hat{\mathcal{L}}_{\widehat{24,278}} \hat{\mathcal{L}}_{\widehat{12}, \mathbf{1 2 3}} \hat{\mathcal{L}}_{\widehat{45}, 470} \hat{\mathcal{L}}_{\widehat{47}, \mathbf{5 8 0}} \hat{\mathcal{L}}_{\widehat{67}, \mathbf{6 9 0}} \\
& \stackrel{\mathcal{P}_{\mathbf{2}} \mathcal{P}_{\mathbf{3}}}{=} \hat{\mathcal{L}}_{\widehat{26}, \mathbf{3 8 9}} \hat{\mathcal{L}}_{\overparen{24,278}} \hat{\mathcal{L}}_{\widehat{45}, 470} \hat{\mathcal{L}}_{\overparen{47}, 580} \hat{\mathcal{L}}_{\widehat{12}, \mathbf{1 2 3}} \hat{\mathcal{L}}_{\widehat{67}, \mathbf{6 9 0}} \\
& \stackrel{\mathcal{T}_{\widehat{4}, \mathbf{3}}}{=} \hat{\mathcal{L}}_{\widehat{26}, \mathbf{3 8 9}} \hat{\mathcal{L}}_{\widehat{46}, \mathbf{5 8 0}} \hat{\mathcal{L}}_{\widehat{34}, \mathbf{4 7 8}} \hat{\mathcal{L}}_{\widehat{14}, \mathbf{2 4 5}} \hat{\mathcal{L}}_{\widehat{12}, \mathbf{1 2 3}} \hat{\mathcal{L}}_{\widehat{67}, 690}
\end{aligned}
$$

\begin{tabular}{|c|c|c|c|c|c|c|c|c|c|c|}
\hline & $\widehat{156}$ & & $\widehat{156}$ & & $\widehat{156}$ & & $\widehat{126}$ & & $\widehat{126}$ & \\
\hline$\widehat{56}$ & $\widehat{356}$ & $\widehat{36}$ & $\widehat{136}$ & $\widehat{34}$ & $\widehat{136}$ & $\widehat{16}$ & $\widehat{146}$ & & $\overline{124}$ & $\widehat{12}$ \\
\hline 12 & $\widehat{236}$ & 23 & $\widehat{346}$ & 34 & $\widehat{134}$ & 12 & $\widehat{134}$ & 23 & $\widehat{145}$ & 12 \\
\hline & 34 & & 234 & & 345 & & 345 & & 345 & \\
\hline
\end{tabular}

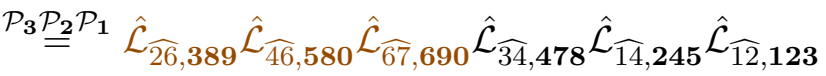

$$
\begin{aligned}
& \stackrel{\mathcal{T}_{\hat{6}, 4}}{=} \hat{\mathcal{L}}_{\widehat{56}, \mathbf{6 9 0}} \hat{\mathcal{L}}_{\widehat{36}, \mathbf{5 8 9}} \hat{\mathcal{L}}_{\widehat{16}, \mathbf{3 5 6}} \hat{\mathcal{L}}_{\widehat{34}, 478} \hat{\mathcal{L}}_{\widehat{14}, \mathbf{2 4 5}} \hat{\mathcal{L}}_{\widehat{12}, \mathbf{1 2 3}} \\
& \stackrel{\mathcal{P}_{3}}{=} \hat{\mathcal{L}}_{\overline{56}, \mathbf{6 9 0}} \hat{\mathcal{L}}_{\widehat{36}, \mathbf{5 8 9}} \hat{\mathcal{L}}_{\widehat{34}, \mathbf{4 7 8}} \hat{\mathcal{L}}_{\widehat{16}, \mathbf{3 5 6}} \hat{\mathcal{L}}_{\widehat{14}, \mathbf{2 4 5}} \hat{\mathcal{L}}_{\widehat{12}, \mathbf{1 2 3}},
\end{aligned}
$$

which is $\hat{\mathcal{L}}_{\bar{\alpha}}=\hat{\mathcal{L}}_{\bar{\omega}} \circ \mathcal{T}_{\tilde{\mathcal{C}}_{e}}$. Hence

$$
\mathcal{T}_{\tilde{\mathcal{C}}_{o}}=\mathcal{T}_{\hat{1}, \mathbf{1}} \mathcal{T}_{\hat{3}, \mathbf{3}} \mathcal{P}_{\mathbf{2}} \mathcal{P}_{\mathbf{5}} \mathcal{T}_{\hat{5}, \mathbf{3}} \mathcal{T}_{\hat{\gamma}, \mathbf{1}} \mathcal{P}_{\mathbf{3}}=\mathcal{P}_{\mathbf{3}} \mathcal{T}_{\hat{6}, \mathbf{4}} \mathcal{P}_{\mathbf{3}} \mathcal{P}_{\mathbf{2}} \mathcal{P}_{\mathbf{1}} \mathcal{T}_{\hat{4}, \mathbf{3}} \mathcal{P}_{\mathbf{2}} \mathcal{P}_{\mathbf{3}} \mathcal{T}_{\hat{2}, \mathbf{4}}=\mathcal{T}_{\tilde{\mathcal{C}}_{e}}
$$

which is the heptagon equation.

Example 4.4. Let $N=5$. Then $\bar{\alpha}=(\widehat{56}, \widehat{36}, \widehat{34}, \widehat{16}, \widehat{14}, \widehat{12}) \in A^{(b)}(6,4)$ and $\bar{\eta}=(\widehat{456}, \widehat{256}, \widehat{236}$, $\widehat{234}) \in A^{(b)}(6,3)$. We thus have the chain

from which we can read off the position indices in the expression

$$
\mathcal{L}_{\bar{\alpha}}=\mathcal{L}_{\widehat{12}, 1} \mathcal{L}_{\widehat{14}, 2} \mathcal{L}_{\widehat{16}, 1} \mathcal{L}_{\widehat{34}, 3} \mathcal{L}_{\widehat{36}, 2} \mathcal{L}_{\widehat{56}, 1}
$$

The Lax system (4.8) consists of the localized pentagon equations

$$
\mathcal{L}_{\widehat{k k_{1}}, \boldsymbol{a}} \mathcal{L}_{\widehat{k k_{3}}, \boldsymbol{a}+\mathbf{1}} \mathcal{L}_{\widehat{k k_{5}}, \boldsymbol{a}}=\left(\mathcal{L}_{\widehat{k k_{4}}, \boldsymbol{a}+1} \mathcal{P}_{\boldsymbol{a}} \mathcal{L}_{\widehat{k k_{2}}, \boldsymbol{a}+1}\right) \circ \mathcal{T}_{\hat{k}},
$$

where $k \in[6], \hat{k}=\left\{k_{1}, \ldots, k_{5}\right\}$ with $k_{1}<\cdots<k_{5}$. The consistency condition is now obtained as follows. We have

$$
\mathcal{L}_{\bar{\alpha}}=\mathcal{L}_{\widehat{12}, 1} \mathcal{L}_{\widehat{14}, \mathbf{2}} \mathcal{L}_{\widehat{16}, 1} \mathcal{L}_{\widehat{34,3}} \mathcal{L}_{\widehat{36}, 2} \mathcal{L}_{\overline{56}, 1} \stackrel{\mathcal{P}_{3}}{=} \mathcal{L}_{\widehat{12}, 1} \mathcal{L}_{\widehat{14}, \mathbf{2}} \mathcal{L}_{\widehat{34}, \mathbf{3}} \mathcal{L}_{\widehat{16}, 1} \mathcal{L}_{\widehat{36}, 2} \mathcal{L}_{\overline{56}, 1}
$$



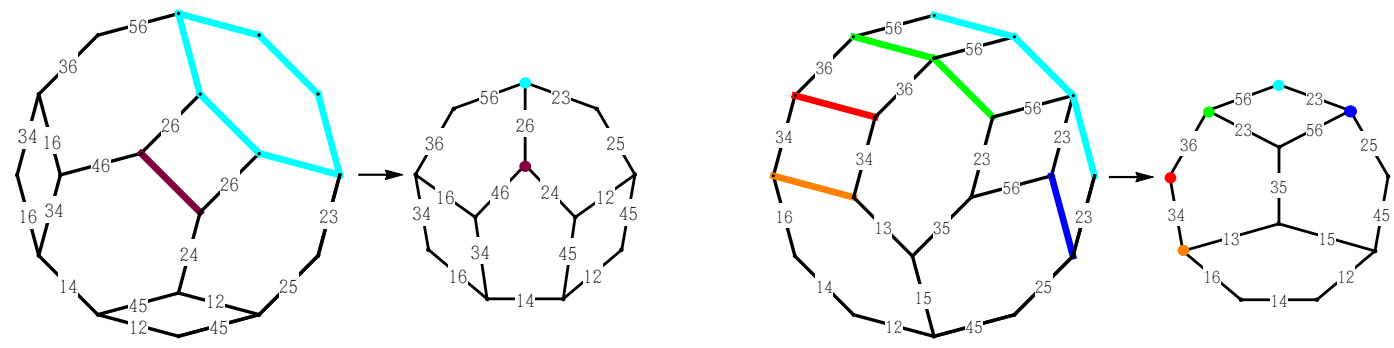

Figure 23. Projection of $T(7,4)$ (Edelman-Reiner polyhedron) to $T(6,3)$ (associahedron). We use complementary labeling.

$$
\begin{aligned}
& \stackrel{\mathcal{T}_{\hat{6}, 1}}{=} \mathcal{L}_{\widehat{12,}, \mathbf{1}} \mathcal{L}_{\widehat{14}, 2} \mathcal{L}_{\widehat{34,3}, 3} \mathcal{L}_{\widehat{46}, 2} \mathcal{P}_{\mathbf{1}} \mathcal{L}_{\widehat{26}, \mathbf{2}} \stackrel{\mathcal{T}_{\widehat{4}, \mathbf{2}}}{=} \mathcal{L}_{\widehat{12,1}, 1} \mathcal{L}_{\widehat{45}, \mathbf{3}} \mathcal{P}_{\mathbf{2}} \mathcal{L}_{\widehat{24}, 3} \mathcal{P}_{\mathbf{1}} \mathcal{L}_{\widehat{26,2}} \\
& \stackrel{\mathcal{P}_{3}}{=} \mathcal{L}_{\widehat{45,3}} \mathcal{L}_{\widehat{12,1}, 1} \mathcal{P}_{\mathbf{2}} \mathcal{L}_{\widehat{24,}, 3} \mathcal{P}_{\mathbf{1}} \mathcal{L}_{\widehat{26,2}}=\mathcal{L}_{\widehat{45,3}, 3} \mathcal{L}_{\widehat{12,1}, 1} \mathcal{P}_{\mathbf{2}} \mathcal{P}_{\mathbf{1}} \mathcal{L}_{\widehat{24,3}, 3} \mathcal{L}_{\widehat{26}, 2} \\
& =\mathcal{L}_{\widehat{45,3}} \mathcal{P}_{\mathbf{2}} \mathcal{P}_{\mathbf{1}} \mathcal{L}_{\widehat{12,2}, 2} \mathcal{L}_{\widehat{24,3}} \mathcal{L}_{\widehat{26,2}} \stackrel{\mathcal{T}_{\hat{2}, \mathbf{1}}}{=} \mathcal{L}_{\widehat{45,3}} \mathcal{P}_{\mathbf{2}} \mathcal{P}_{\mathbf{1}} \mathcal{L}_{\widehat{25,3}} \mathcal{P}_{\mathbf{2}} \mathcal{L}_{\widehat{23,}, \mathbf{3}},
\end{aligned}
$$

which is $\mathcal{L}_{\bar{\alpha}}=\mathcal{L}_{\bar{\omega}} \circ \mathcal{T}_{\tilde{\mathcal{C}}_{o}}$. This yields the left hand side of the hexagon equation (4.4). The right hand side of (4.4) is obtained if we proceed according to the chain $\mathcal{C}_{e}$. The full compositions of maps appearing in this computation can be visualized as maximal chains of $T(6,2)$, which forms a 4-hypercube. For larger values of $N$, the corresponding computation, based on (4.8), becomes very complicated. This is in contrast to the derivation of the consistency condition using the hatted version of the Lax system.

Remark 4.5. The derivation of the hexagon equation for the maps $\mathcal{T}_{\hat{j}}$ in Example 4.4 still works if $\mathcal{P}$ is any braiding map (solution of the Yang-Baxter equation), provided that the following relations hold,

$$
\begin{aligned}
& \mathcal{L}_{a} \mathcal{L}_{a+1} \mathcal{L}_{a}=\mathcal{L}_{a+1} \mathcal{P}_{a} \mathcal{L}_{a+1}, \quad \mathcal{P}_{\boldsymbol{a}} \mathcal{P}_{\boldsymbol{a}+1} \mathcal{P}_{\boldsymbol{a}}=\mathcal{P}_{\boldsymbol{a}+1} \mathcal{P}_{\boldsymbol{a}} \mathcal{P}_{\boldsymbol{a}+1}, \\
& \mathcal{L}_{\boldsymbol{a}} \mathcal{P}_{\boldsymbol{a}+1} \mathcal{P}_{\boldsymbol{a}}=\mathcal{P}_{\boldsymbol{a}+1} \mathcal{P}_{\boldsymbol{a}} \mathcal{L}_{\boldsymbol{a}+1}, \quad \mathcal{P}_{\boldsymbol{a}} \mathcal{P}_{\boldsymbol{a}+1} \mathcal{L}_{\boldsymbol{a}}=\mathcal{L}_{\boldsymbol{a}+1} \mathcal{P}_{\boldsymbol{a}} \mathcal{P}_{\boldsymbol{a}+1}, \\
& \mathcal{P}_{\boldsymbol{a}} \mathcal{L}_{\boldsymbol{b}}=\mathcal{L}_{\boldsymbol{b}} \mathcal{P}_{\boldsymbol{a}} \quad \text { for } \quad|\boldsymbol{a}-\boldsymbol{b}|>1,
\end{aligned}
$$

which determine an extension of the braid group. If $\mathcal{P}$ is the transposition map, as assumed in this work outside of this remark, these relations become identities, with the exception of the first, the pentagon equation.

\subsection{Reductions of polygon equations}

The relation between the Tamari order $T(N, N-2)$ and the $N$-gon equation, together with the projection of Tamari orders defined in Remark 2.17, induces a relation between neighboring polygon equations:

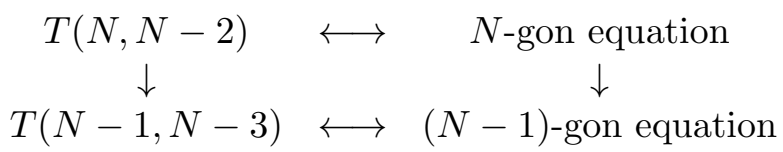

But we have to consider the projection $T(N, N-3) \rightarrow T(N-1, N-4)$ (for $N>4$ ) in order to display the full structure of the corresponding polygon equations. Examples are shown in Figs. 23 and 24. We use the set $\{0,1,2,3,4,5, N-1\}$ instead of $\{1,2,3,4,5,6, N\}$. Unlike the case of simplex equations, there is a substantial difference between odd and even polygon equations. 

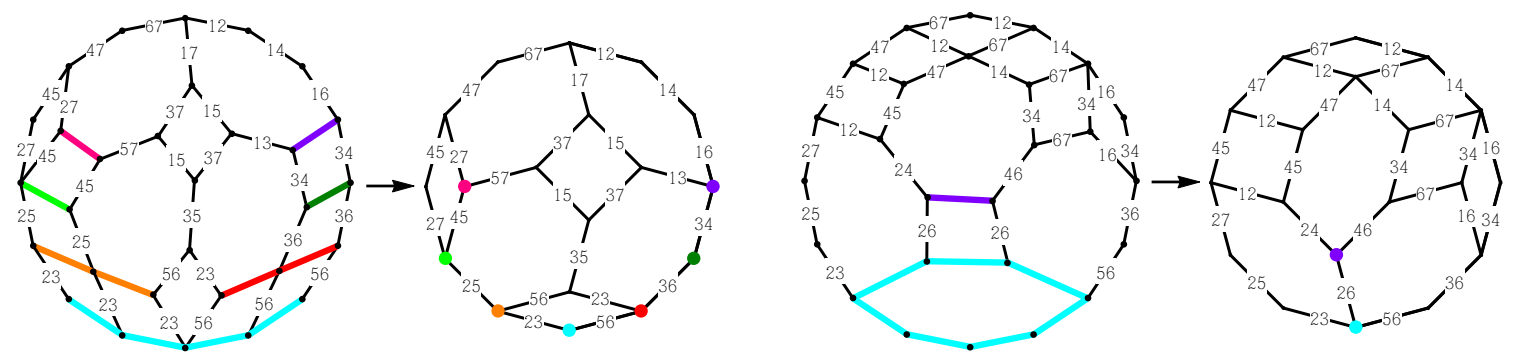

Figure 24. Projection of $T(8,5)$ to $T(7,4)$. As in Fig. 23, the coloring marks those parts of the two Tamari orders that are related by the projection.

Let $N$ be odd, i.e., $N=2 n+1$. Setting

$$
\hat{\mathcal{T}}_{\hat{j}}^{(2 n+1)}:=\hat{\mathcal{T}}_{\hat{j}}^{(2 n)}, \quad j=1, \ldots, 2 n,
$$

and choosing for

$$
\hat{\mathcal{T}}_{\hat{0}}^{(2 n+1)}: \mathcal{U}_{\widehat{02}} \times \mathcal{U}_{\widehat{04}} \times \cdots \times \mathcal{U}_{\widehat{0(2 n)}} \longrightarrow \mathcal{U}_{\widehat{01}} \times \mathcal{U}_{\widehat{03}} \times \cdots \mathcal{U}_{0(\widehat{2 n-1})}
$$

the identity map ${ }^{19}$, it follows that $\hat{\mathcal{T}}_{\hat{j}}^{(2 n+1)}, j=0, \ldots, 2 n$, satisfy the $(2 n+1)$-gon equation.

Example 4.6. The heptagon equation (with labels $0,1, \ldots, 6)$ reads

$$
\hat{\mathcal{T}}_{\hat{0}, \mathbf{1 2 3}}^{(7)} \hat{\mathcal{T}}_{\hat{2}, \mathbf{1 4 5}}^{(7)} \hat{\mathcal{T}}_{\hat{4}, \mathbf{2 4 6}}^{(7)} \hat{\mathcal{T}}_{\hat{6}, \mathbf{3 5 6}}^{(7)}=\hat{\mathcal{T}}_{\hat{5}, \mathbf{3 5 6}}^{(7)} \hat{\mathcal{T}}_{\hat{3}, \mathbf{2 4 5}}^{(7)} \hat{\mathcal{T}}_{\hat{1}, \mathbf{1 2 3}}^{(7)} .
$$

If $\hat{\mathcal{T}}_{\hat{0}}^{(7)}$ is the identity map, this reduces to the hexagon equation

$$
\hat{\mathcal{T}}_{\hat{2}, \mathbf{1 4 5}}^{(6)} \hat{\mathcal{T}}_{\hat{4}, \mathbf{2 4 6}}^{(6)} \hat{\mathcal{T}}_{\hat{6}, \mathbf{3 5 6}}^{(6)}=\hat{\mathcal{T}}_{\hat{5}, \mathbf{3 5 6}}^{(6)} \hat{\mathcal{T}}_{\hat{3}, \mathbf{2 4 5}}^{(6)} \hat{\mathcal{T}}_{\hat{1}, \mathbf{1 2 3}}^{(6)} .
$$

Let now $N$ be even, i.e., $N=2 n$. For $j \in[2 n], j \neq 1$, we have

$$
\hat{\mathcal{T}}_{\hat{j}}^{(2 n)}=\left(u_{\widehat{0 j}}, \mathcal{T}_{\hat{j}}^{(2 n)} \mathcal{P}_{\hat{j}}\right)
$$

where $\hat{j}=\left\{1, j_{2}, \ldots, j_{2 n-1}\right\}$ with $1<j_{2}<\cdots<j_{2 n-1}$, and $u_{\widehat{0 j}} \in \mathcal{U}_{\widehat{0 j}}$. These are maps

$$
\hat{\mathcal{T}}_{\hat{j}}^{(2 n)}: \mathcal{U}_{\widehat{1} j} \times \mathcal{U}_{\widehat{j j_{3}}} \times \cdots \times \mathcal{U}_{\widehat{j j_{2 n-1}}} \longrightarrow \mathcal{U}_{\widehat{0 j}} \times \mathcal{U}_{\widehat{j j_{2}}} \times \mathcal{U}_{\widehat{j j_{4}}} \times \cdots \times \mathcal{U}_{\widehat{j j_{2 n-2}}}
$$

Ignoring the first argument of these maps, the $2 n$-gon equation implies that the resulting maps have to satisfy the $(2 n-1)$-gon equation. Conversely, let $\hat{\mathcal{T}}_{\hat{j}}^{(2 n-1)}, j=2,3, \ldots, 2 n$, satisfy the $(2 n-1)$-gon equation. We extend these maps trivially via

$$
\hat{\mathcal{T}}_{\hat{j}}^{(2 n)}\left(u_{\widehat{1} j}, u_{\widehat{j j_{3}}}, \ldots, u_{\widehat{j j_{2 n-1}}}\right):=\hat{\mathcal{T}}_{\hat{j}}^{(2 n-1)}\left(u_{\widehat{j j_{3}}}, \ldots, u_{\widehat{j j_{2 n-1}}}\right) .
$$

Furthermore, we assume that $\mathcal{U}_{\widehat{01}}=\mathcal{U}_{\widehat{12}}$ and $\mathcal{U}_{\widehat{1(2 j-1)}}=\mathcal{U}_{\widehat{1(2 j)}}, j=2,3, \ldots, n$, and we choose for $\hat{\mathcal{T}}_{\hat{1}}^{(2 n)}$ the identity map. Then, after dropping the first position index in all terms, we find that $\hat{\mathcal{T}}_{\hat{j}}^{(2 n)}, j=1, \ldots, 2 n$, solve the $2 n$-gon equation, with hatted indices shifted by 1 , and with position indices shifted by $n$. In this way, the $2 n$-gon equation reduces to the $(2 n-1)$-gon equation.

\footnotetext{
${ }^{19}$ Recall the identifications made in the definition of $\hat{\mathcal{T}}^{(2 n)}$.
} 
Example 4.7. The octagon equation

$$
\hat{\mathcal{T}}_{\hat{2}, \mathbf{1}, \mathbf{5 , 6 , 7}}^{(8)} \hat{\mathcal{T}}_{\hat{4}, \mathbf{2}, \mathbf{5}, \mathbf{8}, \mathbf{9}}^{(8)} \hat{\mathcal{T}}_{\hat{6}, \mathbf{3}, \mathbf{6}, \mathbf{8}, \mathbf{1 0}}^{(8)} \hat{\mathcal{T}}_{\hat{8}, \mathbf{4}, \mathbf{7}, \mathbf{9}, \mathbf{1 0}}^{(8)}=\hat{\mathcal{T}}_{\hat{7}, \mathbf{4}, \mathbf{7}, \mathbf{9}, \mathbf{1 0}}^{(8)} \hat{\mathcal{T}}_{\hat{5}, \mathbf{3}, \mathbf{6}, \mathbf{8}, \mathbf{9}}^{(8)} \hat{\mathcal{T}}_{\hat{3}, \mathbf{2}, \mathbf{5}, \mathbf{6}, \mathbf{7}}^{(8)} \hat{\mathcal{T}}_{\hat{1}, \mathbf{1 , 2 , 3}, \mathbf{4}}^{(8)}
$$

reduces in the way described above to the heptagon equation (with shifted labels)

$$
\hat{\mathcal{T}}_{\hat{2}, \mathbf{5}, \mathbf{6}, \mathbf{7}}^{(7)} \hat{\mathcal{T}}_{\hat{4}, \mathbf{5 , 8 , 9}}^{(7)} \hat{\mathcal{T}}_{\hat{6}, \mathbf{6 , 8 , 1 0}}^{(7)} \hat{\mathcal{T}}_{\hat{8}, \mathbf{7}, \mathbf{9}, \mathbf{1 0}}^{(7)}=\hat{\mathcal{T}}_{\hat{7}, \mathbf{7 , 9 , 1 0}}^{(7)} \hat{\mathcal{T}}_{\hat{5}, \mathbf{6 , 8 , 9}}^{(7)} \hat{\mathcal{T}}_{\hat{3}, \mathbf{5}, \mathbf{6 , 7}}^{(7)} .
$$

\section{Three color decomposition of simplex equations}

The existence of a decomposition of a Bruhat order into a Tamari order, the corresponding dual Tamari order, and a mixed order, suggests that there should be a way to construct solutions of a simplex equation from solutions of the respective polygon equation and its dual, provided a compatibility condition, associated with the mixed order, is fulfilled. As in Section 4, we associate with $K \in\left(\begin{array}{c}{[N]} \\ N-1\end{array}\right)$ a map $\mathcal{T}_{K}$ and a dual map $\mathcal{S}_{K}$. We set

$$
\mathcal{R}_{K}:=\mathcal{P}^{\prime \prime}\left(\mathcal{T}_{K} \times \mathcal{S}_{K}\right) \mathcal{P}^{\prime}
$$

where $\mathcal{P}^{\prime}, \mathcal{P}^{\prime \prime}$ are compositions of transposition maps achieving the necessary shuffling of $\mathcal{U}_{\vec{P}_{o}(K)}$ and $\mathcal{U}_{\vec{P}_{e}(K)}$, respectively of $\mathcal{U}_{\overleftarrow{P_{o}}(K)}$ and $\mathcal{U}_{\overleftarrow{P_{e}}(K)}$, so that $\mathcal{R}_{K}$ has the correct structure of a simplex $\operatorname{map} \mathcal{U}_{\vec{P}(K)} \rightarrow \mathcal{U}_{\overleftarrow{P}(K)}$. The $(N-1)$-simplex equation then indeed reduces to the $N$-gon equation for $\mathcal{T}_{K}$ and the dual $N$-gon equation for $\mathcal{S}_{K}$, and an additional compatibility condition. This includes one of the results in [57]: special solutions of the 4-simplex equation can be constructed from solutions of the pentagon equation and its dual. The corresponding compatibility condition is $(1.7)$ in [57].

2-simplex and trigon equation. If $N=3$, we consider maps $\mathcal{R}_{i j}: \mathcal{U}_{i} \times \mathcal{U}_{j} \rightarrow \mathcal{U}_{j} \times \mathcal{U}_{i}$, $\mathcal{T}_{i j}: \mathcal{U}_{i} \rightarrow \mathcal{U}_{j}, \mathcal{S}_{i j}: \mathcal{U}_{j} \rightarrow \mathcal{U}_{i}$. In complementary notation, we have, for example, $\mathcal{R}_{\hat{3}}: \mathcal{U}_{\widehat{23}} \times \mathcal{U}_{\widehat{13}} \rightarrow$ $\mathcal{U}_{\widehat{13}} \times \mathcal{U}_{\widehat{23}}, \mathcal{T}_{\hat{3}}: \mathcal{U}_{\widehat{23}} \rightarrow \mathcal{U}_{\widehat{13}}$, and $\mathcal{S}_{\hat{3}}: \mathcal{U}_{\widehat{13}} \rightarrow \mathcal{U}_{\widehat{23}}$. We set $\mathcal{R}_{\hat{k}}=\mathcal{T}_{\hat{k}} \times \mathcal{S}_{\hat{k}}$, hence $\mathcal{R}_{\hat{k}, \boldsymbol{a}}=\mathcal{T}_{\hat{k}, \boldsymbol{a}} \mathcal{S}_{\hat{k}, \boldsymbol{a}+1}$. The 2-simplex equation $\mathcal{R}_{\hat{1}, \mathbf{1}} \mathcal{R}_{\hat{2}, \mathbf{2}} \mathcal{R}_{\hat{3}, \mathbf{1}}=\mathcal{R}_{\hat{3}, \mathbf{2}} \mathcal{R}_{\hat{2}, \mathbf{1}} \mathcal{R}_{\hat{1}, \mathbf{2}}$ then becomes

$$
\mathcal{T}_{\hat{1}, \mathbf{1}} \mathcal{S}_{\hat{1}, \mathbf{2}} \mathcal{T}_{\hat{2}, \mathbf{2}} \mathcal{S}_{\hat{2}, \mathbf{3}} \mathcal{T}_{\hat{3}, \mathbf{1}} \mathcal{S}_{\hat{3}, \mathbf{2}}=\mathcal{T}_{\hat{3}, \mathbf{2}} \mathcal{S}_{\hat{3}, \mathbf{3}} \mathcal{T}_{\hat{2}, \mathbf{1}} \mathcal{S}_{\hat{2}, \mathbf{2}} \mathcal{T}_{\hat{1}, \mathbf{2}} \mathcal{S}_{\hat{1}, \mathbf{3}},
$$

which splits into

$$
\mathcal{T}_{\hat{1}} \mathcal{T}_{\hat{3}}=\mathcal{T}_{\hat{2}}, \quad \mathcal{S}_{\hat{2}}=\mathcal{S}_{\hat{3}} \mathcal{S}_{\hat{1}}, \quad \mathcal{S}_{\hat{1}} \mathcal{T}_{\hat{2}} \mathcal{S}_{\hat{3}}=\mathcal{T}_{\hat{3}} \mathcal{S}_{\hat{2}} \mathcal{T}_{\hat{1}} .
$$

The first two are the trigon equation and its dual. The last equation is an additional condition. A graphical representation of this "decomposition" of the Yang-Baxter equation is shown in Fig. 25.

3-simplex and tetragon equation. For $N=4$, we have $\mathcal{R}_{\hat{1}}: \mathcal{U}_{\widehat{14}} \times \mathcal{U}_{\widehat{13}} \times \mathcal{U}_{\widehat{12}} \rightarrow \mathcal{U}_{\widehat{12}} \times \mathcal{U}_{\widehat{13}} \times \mathcal{U}_{\widehat{14}}$, $\mathcal{T}_{\hat{1}}: \mathcal{U}_{\widehat{14}} \times \mathcal{U}_{\widehat{12}} \rightarrow \mathcal{U}_{\widehat{13}}, \mathcal{S}_{\hat{1}}: \mathcal{U}_{\widehat{13}} \rightarrow \mathcal{U}_{\widehat{12}} \times \mathcal{U}_{\widehat{14}}$, etc. ${ }^{20}$ According to (5.1), we have to set $\mathcal{R}_{\hat{k}}=\mathcal{P}_{\mathbf{1}}\left(\mathcal{T}_{\hat{k}} \times\right.$ $\left.\mathcal{S}_{\hat{k}}\right) \mathcal{P}_{\mathbf{2}}$. This means $\mathcal{R}_{\hat{k}}(u, v, w)=\left(\mathcal{S}_{\hat{k}}(v)_{1}, \mathcal{T}_{\hat{k}}(u, w), \mathcal{S}_{\hat{k}}(v)_{2}\right)$, where $\mathcal{S}_{\hat{k}}(v)=:\left(\mathcal{S}_{\hat{k}}(v)_{1}, \mathcal{S}_{\hat{k}}(v)_{2}\right)$. The 3-simplex equation (3.5) then leads to

$$
\mathcal{T}_{\hat{2}} \mathcal{T}_{\hat{4}, \mathbf{1}}=\mathcal{T}_{\hat{3}} \mathcal{T}_{\hat{1}, \mathbf{2}}, \quad \mathcal{S}_{\hat{1}, \mathbf{1}} \mathcal{S}_{\hat{3}}=\mathcal{S}_{\hat{4}, \mathbf{2}} \mathcal{S}_{\hat{2}}, \quad \mathcal{T}_{\hat{1}, \mathbf{1}} \mathcal{S}_{\hat{2}, \mathbf{2}} \mathcal{T}_{\hat{3}, \mathbf{2}} \mathcal{S}_{\hat{4}, \mathbf{1}}=\mathcal{T}_{\hat{4}, \mathbf{2}} \mathcal{S}_{\hat{3}, \mathbf{1}} \mathcal{T}_{\hat{2}, \mathbf{1}} \mathcal{S}_{\hat{1}, \mathbf{2}} .
$$

The first two equations are the tetragon equation and its dual. The three equations correspond to $B^{(b)}(4,2), B^{(r)}(4,2)$ and $B^{(g)}(4,2)$, respectively, cf. Example 2.14. Also see Fig. 26.

\footnotetext{
${ }^{20}$ In the setting of linear spaces, $\mathcal{T}_{\hat{k}}$ will be a product and $\mathcal{S}_{\hat{k}}$ a coproduct.
} 

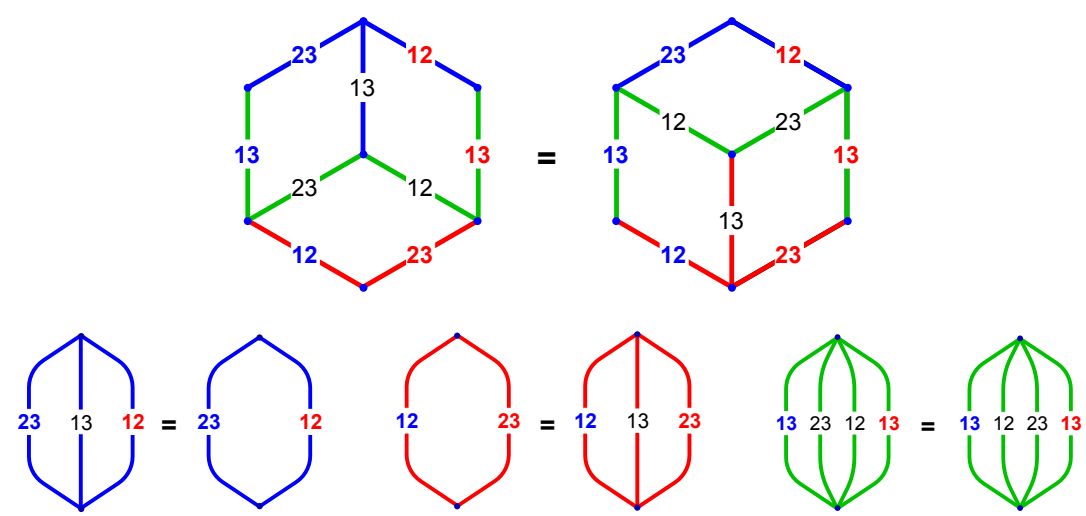

Figure 25. Decomposition of the Yang-Baxter equation viewed on the cube $B(3,0)$ (with complementary edge labeling, but hats omitted). Following the action of the composition of Yang-Baxter maps on the left and the right-hand side of the Yang-Baxter equation, we observe that the action splits as indicated by the three different colors. A set of edges having the same color corresponds to one of three equations, represented by the graphs in the second row (where all vertices connected by edges having a different color have been identified). Blue: trigon equation, red: dual trigon equation, green: compatibility condition. Here, and also in some of the following figures, labels of edges of an initial (final) maximal chain are marked blue (red), which has no further meaning.
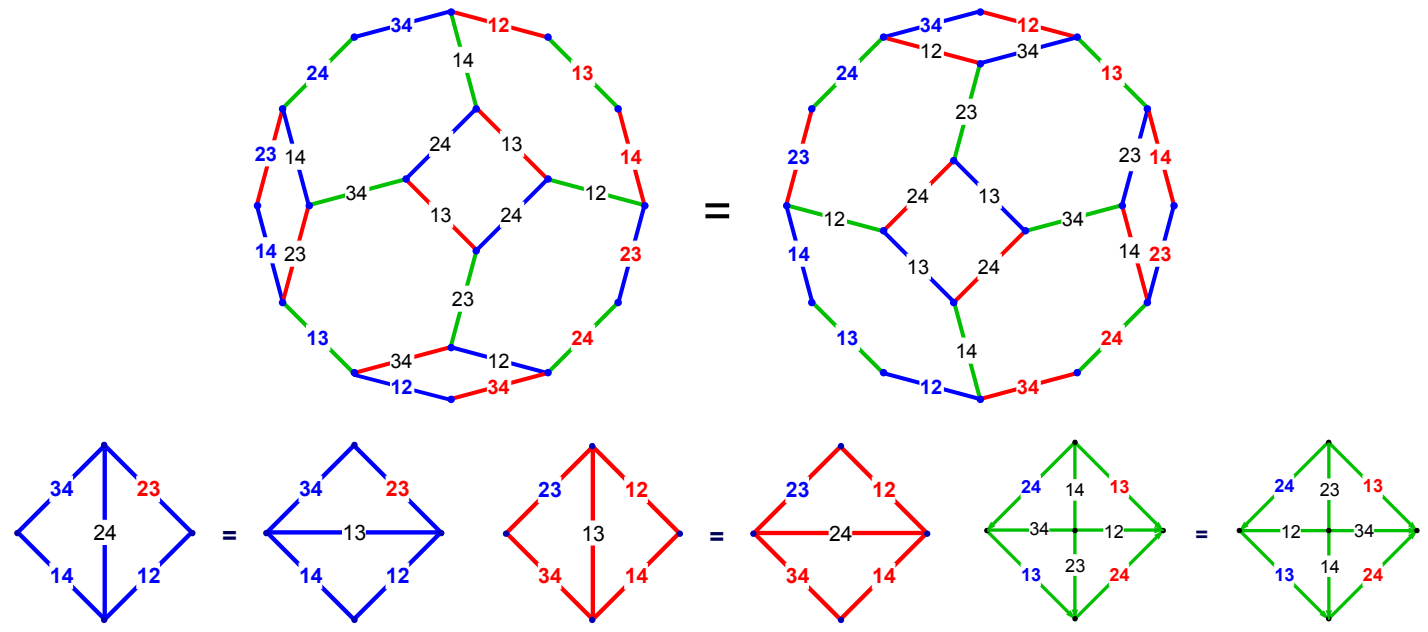

Figure 26. Decomposition of the 3-simplex (tetrahedron, Zamolodchikov) equation, viewed on the permutahedron formed by $B(4,1)$. The second row of figures graphically represents the resulting tetragon equation, dual tetragon equation, and the compatibility condition.

Remark 5.1. By drawing a line through the midpoints of parallel edges, the half-polytopes of $B(4,1)$ in Fig. 26 are mapped to the diagrams in Fig. 5, presented in Fig. 27 in a slightly different way. A tetragon is mapped in this way to a crossing, a hexagon to a node with six legs and a number $\hat{k}$ that associates it with the map $\hat{\mathcal{T}}_{\hat{k}}$. The tetragon equation, its dual and the compatibility equation are then represented by the further graphs in Fig. 27.

4-simplex and pentagon equation. For $N=5$, we have $\mathcal{R}_{\widehat{1}}: \mathcal{U}_{\widehat{15}} \times \mathcal{U}_{\widehat{14}} \times \mathcal{U}_{\widehat{13}} \times \mathcal{U}_{\widehat{12}} \rightarrow$ $\mathcal{U}_{\widehat{12}} \times \mathcal{U}_{\widehat{13}} \times \mathcal{U}_{\widehat{14}} \times \mathcal{U}_{\widehat{15}}, \mathcal{T}_{\hat{1}}: \mathcal{U}_{\widehat{15}} \times \mathcal{U}_{\widehat{13}} \rightarrow \mathcal{U}_{\widehat{12}} \times \mathcal{U}_{\widehat{14}}, \mathcal{S}_{\hat{1}}: \mathcal{U}_{\widehat{14}} \times \mathcal{U}_{\widehat{12}} \rightarrow \mathcal{U}_{\widehat{13}} \times \mathcal{U}_{\widehat{15}}$, etc. We have to set $\mathcal{R}_{\hat{1}}=\mathcal{P}_{\mathbf{2}} \mathcal{T}_{\hat{1}, \mathbf{3}} \mathcal{S}_{\hat{1}, \mathbf{1}} \mathcal{P}_{\mathbf{2}}$, etc., hence $\hat{\mathcal{R}}_{\hat{k}, \mathbf{1 2 3 4}}=\hat{\mathcal{T}}_{\hat{k}, \mathbf{1 3}} \hat{\mathcal{S}}_{\hat{k}, \mathbf{2 4}} \mathcal{P}_{\mathbf{1}, \mathbf{2}} \mathcal{P}_{\mathbf{3 4}}=\hat{\mathcal{T}}_{\hat{k}, \mathbf{1 3}} \mathcal{P}_{\mathbf{1}, \mathbf{2}} \mathcal{P}_{\mathbf{3 4}} \hat{\mathcal{S}}_{\hat{k}, \mathbf{1 3}}$. The 


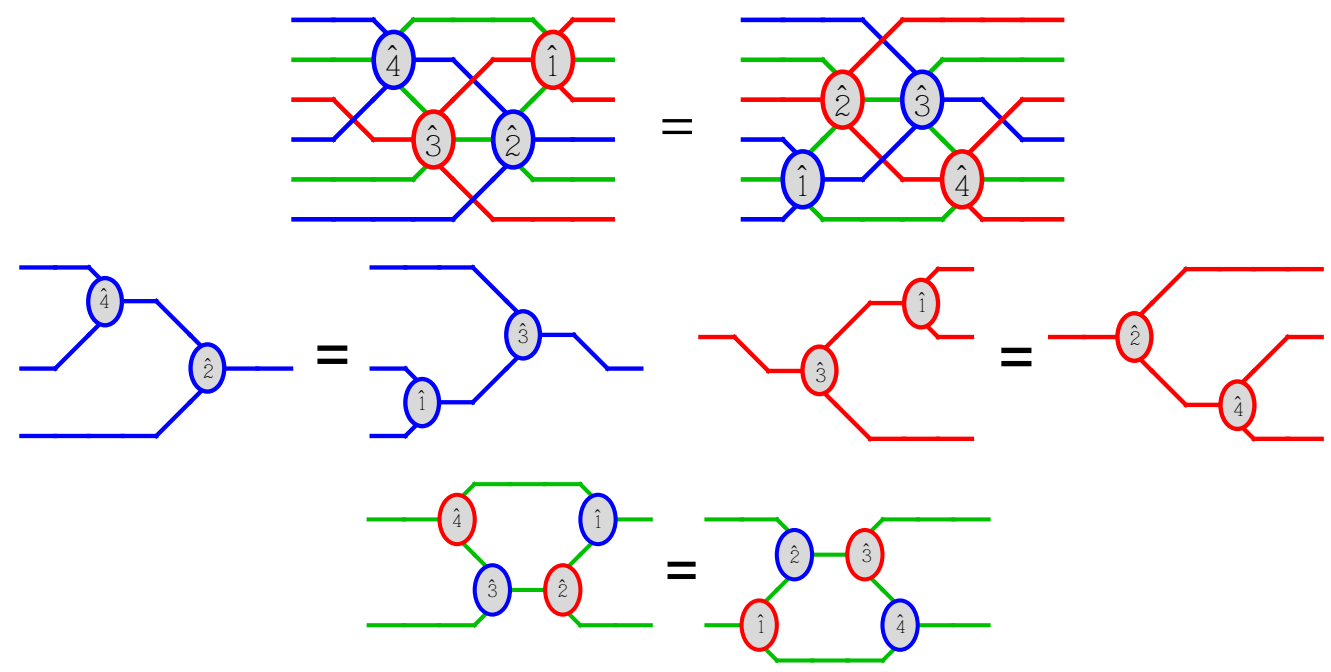

Figure 27. The first line shows the dual of the permutahedron equality in Fig. 26, in the sense of Remark 5.1. The further diagrams represent the three parts that arise from the "decomposition" of the 3 -simplex equation (3.5).

4-simplex equation (3.7) then splits into the pentagon equation (4.3), its dual

$$
\hat{\mathcal{S}}_{\hat{2}, \mathbf{1 2}} \hat{\mathcal{S}}_{\hat{4}, \mathbf{2 3}}=\hat{\mathcal{S}}_{\hat{5}, \mathbf{2 3}} \hat{\mathcal{S}}_{\hat{3}, \mathbf{1 3}} \hat{\mathcal{S}}_{\hat{1}, \mathbf{1 2}}
$$

and the additional condition

$$
\hat{\mathcal{S}}_{\hat{1}, \mathbf{1 2}} \hat{\mathcal{T}}_{\hat{2}, \mathbf{1 3}} \hat{\mathcal{S}}_{\hat{3}, \mathbf{1 4}} \hat{\mathcal{T}}_{\hat{4}, \mathbf{2 4}} \hat{\mathcal{S}}_{\hat{5}, \mathbf{3 4}}=\hat{\mathcal{T}}_{\hat{\bar{S}}, \mathbf{2 4}} \hat{\mathcal{S}}_{\hat{4}, \mathbf{3 4}} \hat{\mathcal{T}}_{\hat{3}, \mathbf{1 4}} \hat{\mathcal{S}}_{\hat{2}, \mathbf{1 2}} \hat{\mathcal{T}}_{\hat{1}, \mathbf{1 3}}
$$

(cf. (1.7) in [57]). In terms of $\mathcal{T}$ and $\mathcal{S}$, we have (4.2) and

$$
\mathcal{S}_{\hat{2}, \mathbf{1}} \mathcal{P}_{\mathbf{2}} \mathcal{S}_{\hat{4}, \mathbf{1}}=\mathcal{S}_{\hat{5}, \mathbf{2}} \mathcal{S}_{\hat{3}, \mathbf{1}} \mathcal{S}_{\hat{1}, \mathbf{2}}, \quad \mathcal{S}_{\hat{1}, \mathbf{1}} \mathcal{T}_{\hat{\mathrm{2}}, \mathbf{2}} \mathcal{S}_{\hat{3}, \mathbf{3}} \mathcal{P}_{\mathbf{1}} \mathcal{T}_{\hat{4}, \mathbf{2}} \mathcal{S}_{\hat{5}, \mathbf{1}} \mathcal{P}_{\mathbf{2}}=\mathcal{P}_{\mathbf{2}} \mathcal{T}_{\hat{5}, \mathbf{3}} \mathcal{S}_{\hat{4}, \mathbf{2}} \mathcal{P}_{\mathbf{3}} \mathcal{T}_{\hat{3}, \mathbf{1}} \mathcal{S}_{\hat{2}, \mathbf{2}} \mathcal{T}_{\hat{1}, \mathbf{3}}
$$

Also see Fig. 28.

5-simplex and hexagon equation. For $N=6$, we have $\mathcal{R}_{\hat{1}}: \mathcal{U}_{\widehat{16}} \times \mathcal{U}_{\widehat{15}} \times \mathcal{U}_{\widehat{14}} \times \mathcal{U}_{\widehat{13}} \times \mathcal{U}_{\widehat{12}} \rightarrow$ $\mathcal{U}_{\widehat{12}} \times \mathcal{U}_{\widehat{13}} \times \mathcal{U}_{\widehat{14}} \times \mathcal{U}_{\widehat{15}} \times \mathcal{U}_{\widehat{16}}, \mathcal{T}_{\hat{1}}: \mathcal{U}_{\widehat{16}} \times \mathcal{U}_{\widehat{14}} \times \mathcal{U}_{\widehat{12}} \rightarrow \mathcal{U}_{\widehat{13}} \times \mathcal{U}_{\widehat{15}}, \mathcal{S}_{\hat{1}}: \mathcal{U}_{\widehat{15}} \times \mathcal{U}_{\widehat{13}} \rightarrow \mathcal{U}_{\widehat{12}} \times \mathcal{U}_{\widehat{14}} \times \mathcal{U}_{\widehat{16}}$ etc. We set $\mathcal{R}_{\hat{k}}=\mathcal{P}_{\mathbf{3}} \mathcal{P}_{\mathbf{1}} \mathcal{P}_{\mathbf{2}}\left(\mathcal{T}_{\hat{k}} \times \mathcal{S}_{\hat{k}}\right) \mathcal{P}_{\mathbf{4}} \mathcal{P}_{\mathbf{2}} \mathcal{P}_{\mathbf{3}}$. The 5 -simplex equation (3.8) then decomposes into the hexagon equation (4.4) for $\mathcal{T}$, the dual hexagon equation

$$
\mathcal{S}_{\hat{1}, \mathbf{1}} \mathcal{S}_{\hat{3}, \mathbf{2}} \mathcal{P}_{\mathbf{3}} \mathcal{S}_{\hat{5}, \mathbf{1}}=\mathcal{P}_{\mathbf{3}} \mathcal{S}_{\hat{6}, 4} \mathcal{S}_{\hat{4}, \mathbf{2}} \mathcal{P}_{\mathbf{1}} \mathcal{S}_{\hat{2}, \mathbf{2}}
$$

and

$$
\mathcal{T}_{\hat{1}, \mathbf{1}} \mathcal{S}_{\hat{2}, \mathbf{3}} \mathcal{T}_{\hat{3}, \mathbf{4}} \mathcal{P}_{\mathbf{6}} \mathcal{P}_{\mathbf{2}} \mathcal{P}_{\mathbf{3}} \mathcal{P}_{\mathbf{2}} \mathcal{S}_{\hat{4}, \mathbf{4}} \mathcal{T}_{\hat{5}, \mathbf{3}} \mathcal{S}_{\hat{6}, \mathbf{1}} \mathcal{P}_{\mathbf{4}} \mathcal{P}_{\mathbf{2}}=\mathcal{P}_{\mathbf{4}} \mathcal{P}_{\mathbf{2}} \mathcal{T}_{\hat{6}, \mathbf{5}} \mathcal{S}_{\hat{5}, \mathbf{3}} \mathcal{T}_{\hat{4}, \mathbf{2}} \mathcal{P}_{\mathbf{5}} \mathcal{P}_{\mathbf{4}} \mathcal{P}_{\mathbf{5}} \mathcal{P}_{\mathbf{1}} \mathcal{S}_{\hat{3}, \mathbf{2}} \mathcal{T}_{\hat{2}, \mathbf{3}} \mathcal{S}_{\hat{1}, \mathbf{5}}
$$

See Fig. 29.

6-simplex and heptagon equation. For $N=7$, we have $\mathcal{R}_{\hat{1}}: \mathcal{U}_{\widehat{17}} \times \mathcal{U}_{\widehat{16}} \times \mathcal{U}_{\widehat{15}} \times \mathcal{U}_{\widehat{14}} \times \mathcal{U}_{\widehat{13}} \times$ $\mathcal{U}_{\widehat{12}} \rightarrow \mathcal{U}_{\widehat{12}} \times \mathcal{U}_{\widehat{13}} \times \mathcal{U}_{\widehat{14}} \times \mathcal{U}_{\widehat{15}} \times \mathcal{U}_{\widehat{16}} \times \mathcal{U}_{\widehat{17}}, \mathcal{T}_{\hat{1}}: \mathcal{U}_{\widehat{17}} \times \mathcal{U}_{\widehat{15}} \times \mathcal{U}_{\widehat{13}} \rightarrow \mathcal{U}_{\widehat{12}} \times \mathcal{U}_{\widehat{14}} \times \mathcal{U}_{\widehat{16}}, \mathcal{S}_{\hat{1}}: \mathcal{U}_{\widehat{16}} \times \mathcal{U}_{\widehat{14}} \times \mathcal{U}_{\widehat{12}} \rightarrow$ $\mathcal{U}_{\widehat{13}} \times \mathcal{U}_{\widehat{15}} \times \mathcal{U}_{\widehat{17}}$, etc. We set $\mathcal{R}_{\hat{k}}=\mathcal{P}_{\mathbf{4}} \mathcal{P}_{\mathbf{2}} \mathcal{P}_{\mathbf{3}}\left(\mathcal{T}_{\hat{k}} \times \mathcal{S}_{\hat{k}}\right) \mathcal{P}_{\mathbf{3}} \mathcal{P}_{\mathbf{2}} \mathcal{P}_{\mathbf{4}}=\mathcal{P}_{\mathbf{4}} \mathcal{P}_{\mathbf{2}} \mathcal{P}_{\mathbf{3}} \mathcal{T}_{\hat{k}, \mathbf{1}} \mathcal{S}_{\hat{k}, \mathbf{4}} \mathcal{P}_{\mathbf{3}} \mathcal{P}_{\mathbf{2}} \mathcal{P}_{\mathbf{4}}$. The 6 -simplex equation (3.9) then decomposes into the heptagon equation (4.6), respectively (4.7), and the dual heptagon equation

$$
\mathcal{S}_{\hat{2}, \mathbf{1}} \mathcal{P}_{\mathbf{3}} \mathcal{P}_{\mathbf{4}} \mathcal{P}_{5} \mathcal{S}_{\hat{4}, \mathbf{2}} \mathcal{P}_{\mathbf{4}} \mathcal{P}_{\mathbf{3}} \mathcal{S}_{\hat{6}, \mathbf{1}} \mathcal{P}_{\mathbf{3}}=\mathcal{P}_{\mathbf{3}} \mathcal{S}_{\hat{7}, \mathbf{4}} \mathcal{S}_{\hat{5}, \mathbf{2}} \mathcal{P}_{4} \mathcal{P}_{\mathbf{1}} \mathcal{S}_{\hat{3}, \mathbf{2}} \mathcal{S}_{\hat{1}, \mathbf{4}}
$$



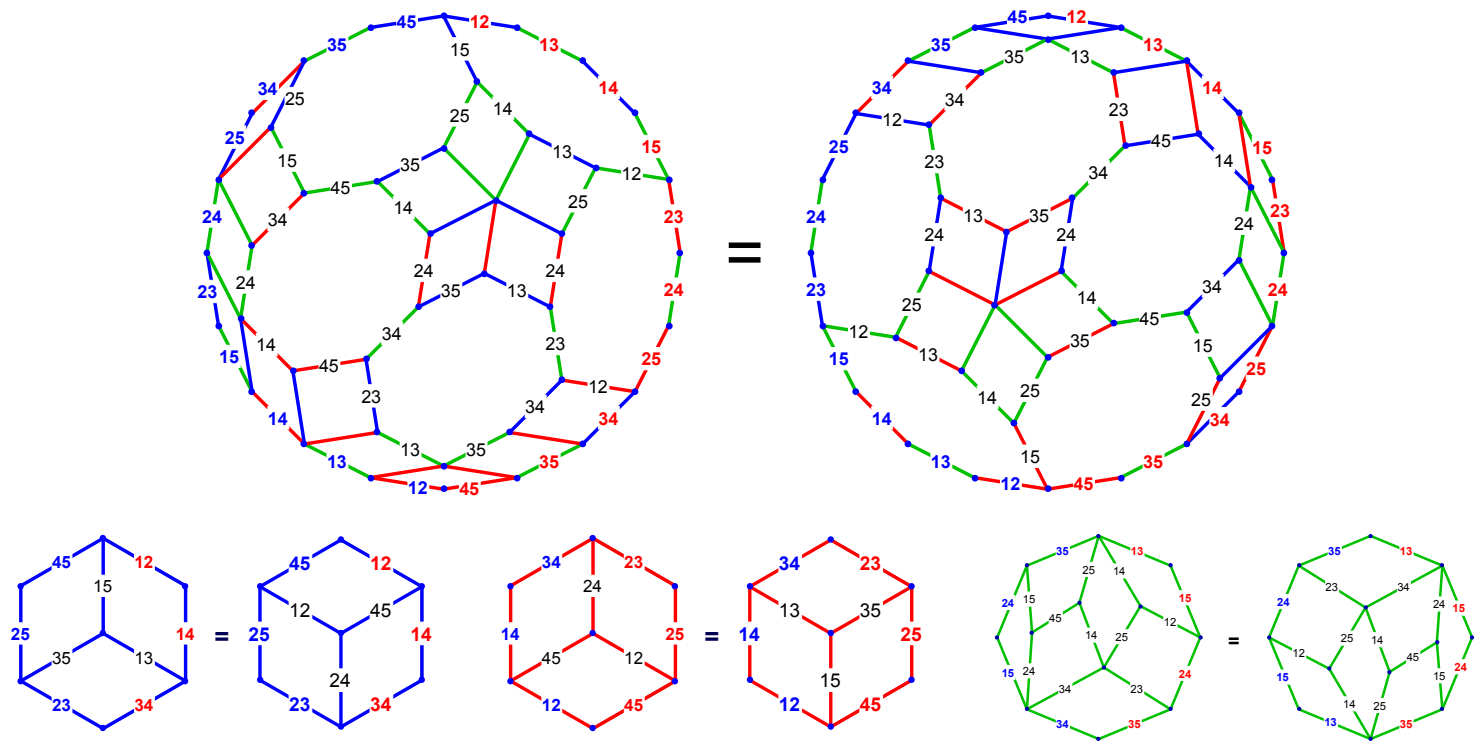

Figure 28. Decomposition of the 4-simplex (Bazhanov-Stroganov) equation, viewed on the FelsnerZiegler polyhedron formed by $B(5,2)$. The resulting pentagon, dual pentagon and compatibility equations are represented by the graphs in the second row.
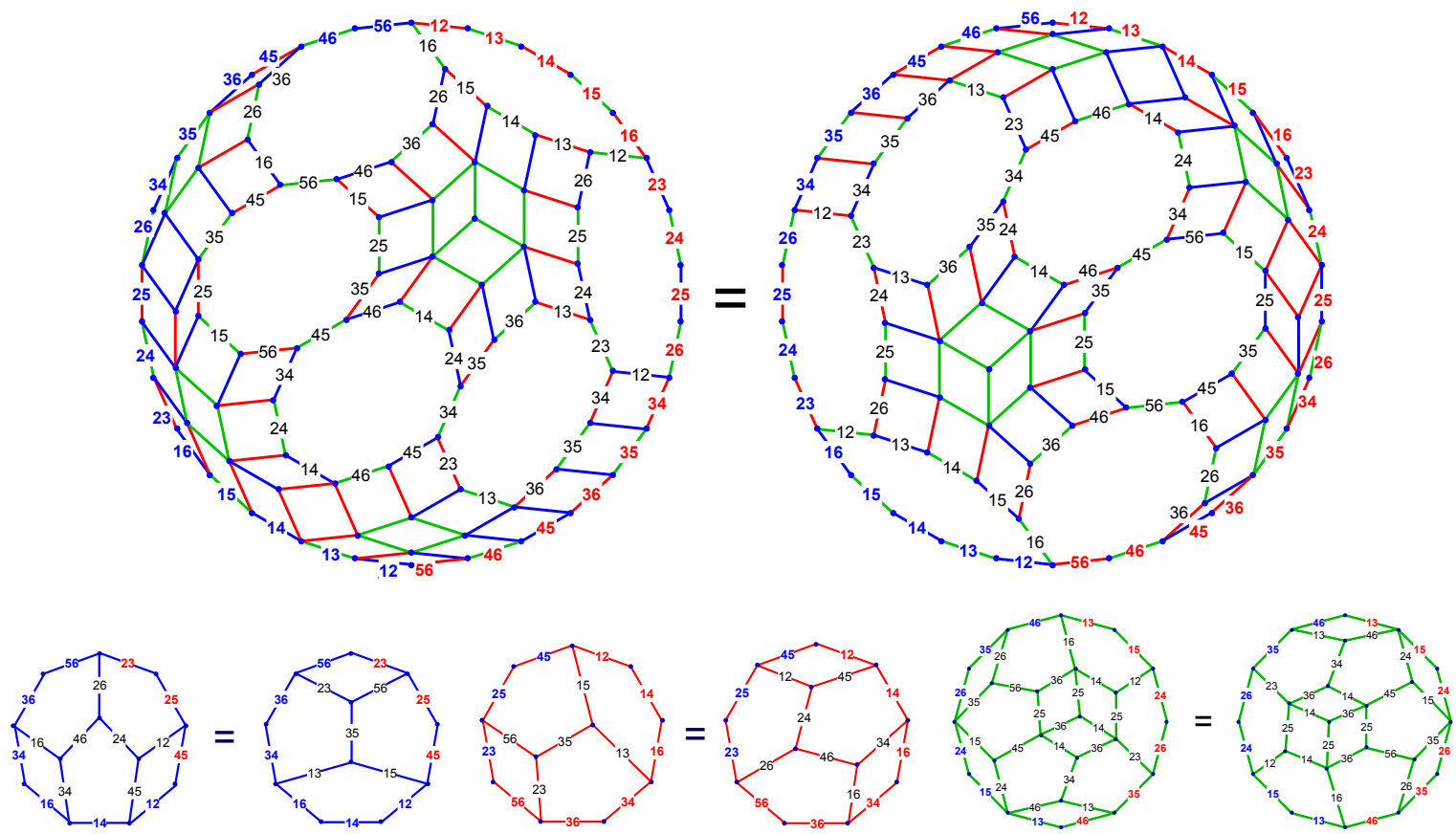

Figure 29. Decomposition of the 5 -simplex equation on the polyhedron formed by $B(6,3)$. The resulting hexagon, dual hexagon and compatibility equations are graphically represented in the second row.

respectively,

$$
\hat{\mathcal{S}}_{\hat{2}, \mathbf{1 2 3}} \hat{\mathcal{S}}_{\hat{4}, \mathbf{2 4 5}} \hat{\mathcal{S}}_{\hat{6}, \mathbf{3 5 6}}=\hat{\mathcal{S}}_{\hat{7}, \mathbf{3 5 6}} \hat{\mathcal{S}}_{\hat{5}, \mathbf{2 4 6}} \hat{\mathcal{S}}_{\hat{3}, \mathbf{1 4 5}} \hat{\mathcal{S}}_{\hat{1}, \mathbf{1 2 3}},
$$

and the compatibility condition

$$
\begin{aligned}
& \mathcal{T}_{\hat{1}, \mathbf{1}} \mathcal{S}_{\hat{2}, \mathbf{3}} \mathcal{P}_{\mathbf{2}} \mathcal{P}_{\mathbf{1}} \mathcal{P}_{\mathbf{3}} \mathcal{T}_{\hat{3}, \mathbf{5}} \mathcal{P}_{\mathbf{7}} \mathcal{P}_{\mathbf{8}} \mathcal{P}_{\mathbf{4}} \mathcal{P}_{\mathbf{3}} \mathcal{S}_{\hat{4}, \mathbf{5}} \mathcal{P}_{\mathbf{7}} \mathcal{P}_{\mathbf{4}} \mathcal{P}_{\mathbf{3}} \mathcal{P}_{\mathbf{2}} \mathcal{T}_{\hat{5}, \mathbf{5}} \mathcal{P}_{\mathbf{7}} \mathcal{S}_{\hat{6}, \mathbf{3}} \mathcal{P}_{\mathbf{5}} \mathcal{T}_{\hat{7}, \mathbf{1}} \mathcal{P}_{\mathbf{3}} \mathcal{P}_{\mathbf{4}} \mathcal{P}_{\mathbf{5}} \mathcal{P}_{\mathbf{6}} \mathcal{P}_{\mathbf{2}} \\
& =\mathcal{P}_{\mathbf{7}} \mathcal{P}_{\mathbf{5}} \mathcal{P}_{\mathbf{3}} \mathcal{P}_{\mathbf{4}} \mathcal{P}_{\mathbf{5}} \mathcal{P}_{\mathbf{6}} \mathcal{P}_{\mathbf{2}} \mathcal{S}_{\hat{7}, \mathbf{7}} \mathcal{T}_{\hat{6}, \mathbf{5}} \mathcal{P}_{\mathbf{7}} \mathcal{P}_{\mathbf{8}} \mathcal{P}_{\mathbf{6}} \mathcal{S}_{\hat{\mathrm{5}}, \mathbf{3}} \mathcal{P}_{\mathbf{5}} \mathcal{P}_{\mathbf{6}} \mathcal{P}_{\mathbf{2}} \mathcal{P}_{\mathbf{1}} \mathcal{T}_{\hat{4}, \mathbf{3}} \mathcal{P}_{\mathbf{5}} \mathcal{P}_{\mathbf{6}} \mathcal{P}_{\mathbf{7}} \mathcal{P}_{\mathbf{2}} \mathcal{S}_{\hat{3}, \mathbf{3}} \mathcal{T}_{\hat{2}, \mathbf{5}} \mathcal{S}_{\hat{1}, \mathbf{7}}
\end{aligned}
$$



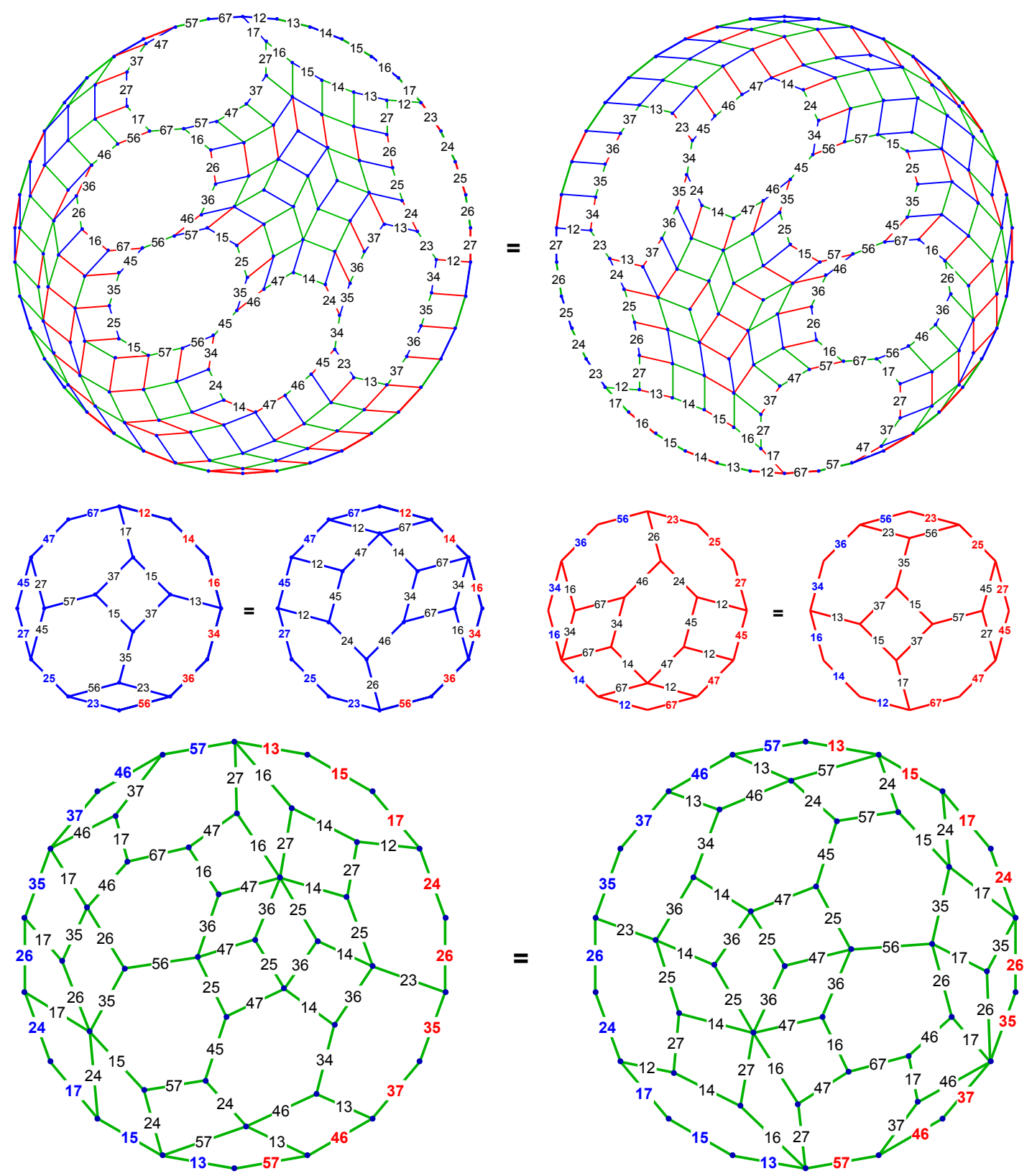

Figure 30. Decomposition of the 6 -simplex equation on the polyhedron formed by $B(7,4)$. The resulting heptagon, dual heptagon and compatibility equations are represented by the graphs in the last two rows.

respectively

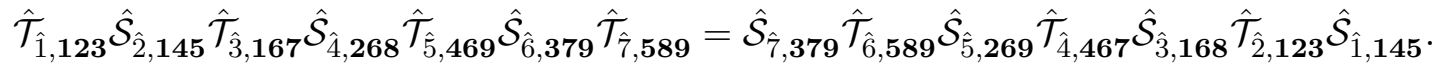

See Fig. 30.

\section{Conclusions}

The main result of this work is the existence of an infinite family of "polygon equations" that generalize the pentagon equation in very much the same way as the simplex equations generalize the Yang-Baxter equation. The underlying combinatorial structure in case of simplex equations 
is given by (higher) Bruhat orders [78, 79, 80]. Underlying the polygon equations are (higher) Tamari orders.

We also introduced a visualization of simplex as well as polygon equations as deformations of maximal chains of posets forming 1-skeletons of polyhedra. This geometrical representation revealed various deep relations between such equations.

An intermediate result, worth to highlight, is the (three color) decomposition in Section 2.2 of any (higher) Bruhat order into a (higher) Tamari order, the corresponding dual Tamari order, and a "mixed order". From this we recovered a relation between the pentagon and the 4-simplex equation, observed in [57]. Moreover, we showed that this is just a special case of a relation between any simplex equation and its associated polygon equation. Another (more profound) observation made in [57] concerns a relation between the pentagon equation and the 3 -simplex equation. This seems not to have a corresponding generalization.

Further exploration of the higher polygon equations is required. We expect that they will play a role in similarly diverse problems as the pentagon equation does. A major task will be the search for relevant solutions in suitable frameworks. Such a framework could be the KP hierarchy, since a subclass of its soliton solutions realizes higher Tamari orders [24, 25].

\section{Appendix A: A different view of simplex and polygon equations}

Let $\mathcal{B}$ be a monoid and $N>2$ an integer. With each $J \in\left(\begin{array}{c}{[N]} \\ N-2\end{array}\right)$, we associate an invertible element $L_{J}$ of $\mathcal{B}$, and with each $K \in\left(\begin{array}{c}{[N]} \\ N-1\end{array}\right)$, we associate elements $R_{K}, R_{K}^{\prime}$. They shall be subject to the conditions ([25], also see [20] for a related structure)

$$
\begin{aligned}
& L_{J} L_{J^{\prime}}=L_{J^{\prime}} L_{J} \quad \text { if } \quad E(J) \cap E\left(J^{\prime}\right)=\varnothing, \\
& L_{J} R_{K}=R_{K} L_{J}, \quad L_{J} R_{K}^{\prime}=R_{K}^{\prime} L_{J} \quad \text { if } \quad J \notin P(K), \\
& L_{\vec{P}(K)} R_{K}^{\prime}=R_{K} L_{\overleftarrow{P}(K)},
\end{aligned}
$$

where

$$
L_{\vec{P}(K)}=L_{K \backslash\left\{k_{N-1}\right\}} \cdots L_{K \backslash\left\{k_{1}\right\}}, \quad L_{\overleftarrow{P}(K)}=L_{K \backslash\left\{k_{1}\right\}} \cdots L_{K \backslash\left\{k_{N-1}\right\}}
$$

and $K=\left\{k_{1}, \ldots, k_{N-1}\right\}$ with $k_{1}<k_{2}<\cdots<k_{N-1}$. For any sequence $\rho=\left(J_{1}, \ldots, J_{r}\right)$, let $L_{\rho}=L_{J_{1}} \cdots L_{J_{r}}$. If $\rho \in A(N, N-2)$, then $L_{\rho^{\prime}}=L_{\rho}$ for any $\rho^{\prime} \in[\rho]$, according to (A.1). Hence $L_{\rho}$ represents $[\rho]$.

Proposition A.1.

$$
R_{\vec{P}([N])}=R_{\overleftarrow{P}([N])} \quad \Longleftrightarrow \quad R_{\vec{P}([N])}^{\prime}=R_{\overleftarrow{P}([N])}^{\prime}
$$

Proof. We follow the two maximal chains (2.1) of $B(N, N-2)$. Let $\alpha$ be the lexicographical order on $\left({ }_{N-2}^{[N]}\right)$, and $\omega$ the reverse lexicographical order. Let us start with $L_{\alpha} R_{\vec{P}([N])}^{\prime}=$ $L_{\alpha} R_{\hat{N}}^{\prime} \cdots R_{\hat{1}}^{\prime}$ and move all $L_{J}, J \in P(\hat{N})$, to consecutive positions in $L_{\alpha}$, using (A.1). Using (A.2), we commute $R_{\hat{N}}^{\prime}$ to the left until we have the substring $L_{\vec{P}(\hat{N})} R_{\hat{N}}^{\prime}$. Then we use (A.3) to replace this by $R_{\hat{N}} L \overleftarrow{P}(\hat{N})$. Using (A.2) again, we commute $R_{\hat{N}}$ to the left of all $L$ 's, thus obtaining $R_{\hat{N}} L_{\rho_{1}} R_{\widehat{N-1}}^{\prime} \cdots R_{\hat{1}}^{\prime}$. Continuing in this way, we finally get $L_{\alpha} R_{\vec{P}([N])}^{\prime}=R_{\vec{P}([N])} L_{\omega}$. For the second maximal chain of $B(N, N-2)$, we obtain $L_{\alpha} R_{\overleftarrow{P}([N])}^{\prime}=R_{\overleftarrow{P}([N])} L_{\omega}$ correspondingly. Since $L_{\alpha}$ and $L_{\omega}$ are invertible (since we assume that the $L$ 's are invertible), the statement of the proposition follows. 
The proposition says that the elements $R_{K}, K \in\left(\begin{array}{c}{[N]} \\ N-1\end{array}\right)$, satisfy (the algebraic version of) the $(N-1)$-simplex equation if and only if this is so for $R_{K}^{\prime}, K \in\left(\begin{array}{c}{[N]} \\ N-1\end{array}\right)$. Choosing for all $R_{K}^{\prime}$ the identity element of $\mathcal{B},\left(\right.$ A.3) becomes the Lax system $L_{\vec{P}(K)}=R_{K} L \overleftarrow{P}(K)$.

Example A.2. For $N=3$, the Lax system reads $L_{i} L_{j}=R_{i j} L_{j} L_{i}, 1 \leq i<j \leq 3$, so that $R_{i j}=L_{i} L_{j} L_{i}^{-1} L_{j}^{-1}=:\left[L_{i}, L_{j}\right]$ is a commutator in a group. The condition (A.1) is empty and (A.2) requires $\left[\left[L_{i}, L_{j}\right], L_{k}\right]=e$ for $i<j, k \neq i, j$, where $e$ is the identity element. Hence, if $G$ is the group $\left\langle g_{1}, g_{2}, g_{3} \mid\left[\left[g_{1}, g_{2}\right], g_{3}\right]=\left[\left[g_{2}, g_{3}\right], g_{1}\right]=\left[\left[g_{1}, g_{3}\right], g_{2}\right]=e\right\rangle$, then Proposition A.1 implies that $R_{i j}:=\left[g_{i}, g_{j}\right], i<j$, satisfy the Yang-Baxter equation. If $G$ is abelian, then $R_{i j}=e$.

We are led to the following by the three color decomposition. Let us keep (A.1), but replace (A.2) and (A.3) by

$$
\begin{aligned}
& L_{J} T_{K}=T_{K} L_{J}, \quad L_{J} T_{K}^{\prime}=T_{K}^{\prime} L_{J} \quad \text { if } \quad J \notin P(K) \\
& L_{\overrightarrow{P_{o}}(K)} T_{K}^{\prime}=T_{K} L_{\overleftarrow{P_{e}}(K)} .
\end{aligned}
$$

Then we have

$$
T_{\vec{P}_{o}([N])}=T_{\overleftarrow{P}_{e}([N])} \quad \Longleftrightarrow \quad T_{\vec{P}_{o}([N])}^{\prime}=T_{\overleftarrow{P}_{e}([N])}^{\prime}
$$

The proof is analogous to that of Proposition A.1, but here we start with $L_{\alpha^{(b)}} T_{\vec{P}_{o}([N])}^{\prime}$. Choosing for $T_{K}^{\prime}$ the identity element, we have the Lax system $L_{\overrightarrow{P_{o}}(K)}=T_{K} L_{\overleftarrow{P_{e}}(K)}$ for (an algebraic version of) the $N$-gon equation.

Let us now keep (A.1), but replace (A.2) and (A.3) by

$$
\begin{aligned}
& L_{J} S_{K}=S_{K} L_{J}, \quad L_{J} S_{K}^{\prime}=S_{K}^{\prime} L_{J} \quad \text { if } \quad J \notin P(K), \\
& L_{\vec{P}_{e}(K)} S_{K}^{\prime}=S_{K} L_{\overleftarrow{P}_{o}(K)}
\end{aligned}
$$

Then we have

$$
S_{\vec{P}_{e}([N])}=S_{\overleftarrow{P_{o}}([N])} \quad \Longleftrightarrow \quad S_{\vec{P}_{e}([N])}^{\prime}=S_{\overleftarrow{P_{o}}([N])}^{\prime}
$$

Here the proof starts with $L_{\alpha^{(r)}} S_{\vec{P}_{e}([N])}^{\prime}$. Choosing for $T_{K}^{\prime}$ the identity element, we have the Lax system $L_{\vec{P}_{e}(K)}=S_{K} L_{\overleftarrow{P}_{o}(K)}$ for (an algebraic version of) the dual $N$-gon equation.

Next, let (A.1), (A.4) and (A.5) hold, and in addition

$$
L_{\vec{P}_{e}(K)} T_{K}^{\prime}=T_{K} L_{\overleftarrow{P}_{o}(K)}, \quad L_{\vec{P}_{o}(K)} S_{K}^{\prime}=S_{K} L_{\overleftarrow{P_{e}}(K)}
$$

For odd $N$, the mixed equation reads

$$
S_{\hat{N}} T_{\widehat{N-1}} \cdots T_{\hat{2}} S_{\hat{1}}=T_{\hat{1}} S_{\hat{2}} \cdots S_{\widehat{N-1}} T_{\hat{N}},
$$

while for even $N$ is has the form

$$
S_{\hat{N}} T_{\widehat{N-1}} \cdots S_{\hat{2}} T_{\hat{1}}=S_{\hat{1}} T_{\hat{2}} \cdots S_{\widehat{N-1}} T_{\hat{N}}
$$

We find that the mixed equation holds for $S_{K}, T_{K}$ if and only if it holds for $S_{K}^{\prime}, T_{K}^{\prime}$. The proof starts with $L_{\alpha(g)} S_{\hat{N}}^{\prime} T_{\widehat{N-1}}^{\prime} \cdots T_{\hat{2}}^{\prime} S_{\hat{1}}^{\prime}$ for odd $N$, and correspondingly for even $N$. 
Remark A.3. In the present framework, the pentagon equation reads ${ }^{21}$

$$
T_{1,2,3,4} T_{1,2,4,5} T_{2,3,4,5}=T_{1,3,4,5} T_{1,2,3,5}
$$

Here we inserted commas, which we mostly omitted before. We translate the labels as follows. If a label $i_{1}, i_{2}, i_{3}, i_{4}$ contains a pair with $i_{r+1}=i_{r}+2$ (higher shifts do not appear), then we make the substitution $i_{r}, i_{r+1} \mapsto i_{r}\left(i_{r}+1\right), i_{r+1}$, where $i_{r}\left(i_{r}+1\right)$ is understood as a two-digit expression. Finally we drop the very last index of the resulting new label. If there is no index pair of the above kind in a label, we keep the label, but also drop the very last index. This translates the above pentagon equation to

$$
T_{1,2,3} T_{1,23,4} T_{2,3,4}=T_{12,3,4} T_{1,2,34}
$$

In this form the pentagon equation shows up in Drinfeld's theory of associators (see, e.g., [4, 36]). In the same way, the associated (tetragon) Lax equation $L_{1,2,3} L_{1,3,4} T_{1,2,3,4}^{\prime}=L_{2,3,4} L_{1,2,4}$ becomes

$$
L_{1,2} L_{12,3} T_{1,2,3}^{\prime}=L_{2,3} L_{1,23},
$$

which becomes the twist equation in the context of associators (see, e.g., equation (2) in [4]). Furthermore, the 3 -simplex equation in the present framework is $R_{1,2,3} R_{1,2,4} R_{1,3,4} R_{2,3,4}=$ $R_{2,3,4} R_{1,3,4} R_{1,2,4} R_{1,2,3}$, which translates to

$$
R_{1,2} R_{1,23} R_{12,3} R_{2,3}=R_{2,3} R_{12,3} R_{1,23} R_{1,2}
$$

and the hexagon equation $T_{1,2,3,4,5} T_{1,2,3,5,6} T_{1,3,4,5,6}=T_{2,3,4,5,6} T_{1,2,4,5,6} T_{1,2,3,4,6}$ becomes

$$
T_{1,2,3,4} T_{1,2,34,5} T_{12,3,4,5}=T_{2,3,4,5} T_{1,23,4,5} T_{1,2,3,45}
$$

and so forth.

\section{Acknowledgments}

We have to thank an anonymous referee for comments that led to some corrections in our previous version of Section 2.2.

\section{References}

[1] Adler V.E., Bobenko A.I., Suris Yu.B., Geometry of Yang-Baxter maps: pencils of conics and quadrirational mappings, Comm. Anal. Geom. 12 (2004), 967-1007, math.QA/0307009.

[2] Aitchison I.R., The geometry of oriented cubes, arXiv:1008.1714.

[3] Alekseev A., Enriquez B., Torossian C., Drinfeld associators, braid groups and explicit solutions of the Kashiwara-Vergne equations, Publ. Math. Inst. Hautes Études Sci. (2010), 143-189, arXiv:0903.4067.

[4] Alekseev A., Torossian C., The Kashiwara-Vergne conjecture and Drinfeld's associators, Ann. of Math. 175 (2012), 415-463, arXiv:0802.4300.

[5] Andersen J.E., Kashaev R., A TQFT from quantum Teichmüller theory, Comm. Math. Phys. 330 (2014), 887-934, arXiv:1109.6295.

[6] Baaj S., Skandalis G., Unitaires multiplicatifs et dualité pour les produits croisés de $C^{*}$-algèbres, Ann. Sci. École Norm. Sup. (4) 26 (1993), 425-488.

[7] Baaj S., Skandalis G., Transformations pentagonales, C. R. Acad. Sci. Paris Sér. I Math. 327 (1998), 623-628.

\footnotetext{
${ }^{21}$ This corresponds to the pentagon equation (4.2) without the position indices and with reversion of the order on both sides.
} 
[8] Baaj S., Skandalis G., Unitaires multiplicatifs commutatifs, C. R. Math. Acad. Sci. Paris 336 (2003), 299-304.

[9] Baez J.C., Neuchl M., Higher-dimensional algebra. I. Braided monoidal 2-categories, Adv. Math. 121 (1996), 196-244, q-alg/9511013.

[10] Bar-Natan D., On associators and the Grothendieck-Teichmuller group. I, Selecta Math. (N.S.) 4 (1998), 183-212, q-alg/9606021.

[11] Baxter R.J., Partition function of the eight-vertex lattice model, Ann. Physics 70 (1972), 193-228.

[12] Baxter R.J., Solvable eight-vertex model on an arbitrary planar lattice, Philos. Trans. Roy. Soc. London Ser. A 289 (1978), 315-346.

[13] Baxter R.J., On Zamolodchikov's solution of the tetrahedron equations, Comm. Math. Phys. 88 (1983), $185-205$.

[14] Baxter R.J., The Yang-Baxter equations and the Zamolodchikov model, Phys. D 18 (1986), 321-347.

[15] Bazhanov V.V., Lukyanov S.L., Zamolodchikov A.B., Integrable structure of conformal field theory. III. The Yang-Baxter relation, Comm. Math. Phys. 200 (1999), 297-324, hep-th/9805008.

[16] Bazhanov V.V., Mangazeev V.V., Okada Y., Sergeev S.B., An elliptic parameterisation of the Zamolodchikov model, Nuclear Phys. B 871 (2013), 127-144, arXiv:1212.6800.

[17] Bazhanov V.V., Reshetikhin N.Yu., Remarks on the quantum dilogarithm, J. Phys. A: Math. Gen. 28 (1995), 2217-2226.

[18] Bazhanov V.V., Stroganov Yu.G., Conditions of commutativity of transfer matrices on a multidimensional lattice, Theoret. and Math. Phys. 52 (1982), 685-691.

[19] Biedenharn L.C., Louck J.D., Angular momentum in quantum physics, Encyclopedia of Mathematics and its Applications, Vol. 8, Addison-Wesley, Reading, MA, 1981.

[20] Bytsko A., Volkov A., Tetrahedron equation and cyclic quantum dilogarithm identities, Int. Math. Res. Not. 2015 (2015), 1075-1100, arXiv:1304.1641.

[21] Carter J.S., Saito M., Knotted surfaces and their diagrams, Mathematical Surveys and Monographs, Vol. 55, Amer. Math. Soc., Providence, RI, 1998.

[22] Cartier P., Développements récents sur les groupes de tresses. Applications à la topologie et à l'algèbre, Astérisque (1990), exp. no. 716, 17-67.

[23] Cherednik I.V., Factorizing particles on a half-line and root systems, Theoret. and Math. Phys. 61 (1984), 977-983.

[24] Dimakis A., Müller-Hoissen F., KP line solitons and Tamari lattices, J. Phys. A: Math. Theor. 44 (2011), 025203, 49 pages, arXiv:1009.1886.

[25] Dimakis A., Müller-Hoissen F., KP solitons, higher Bruhat and Tamari orders, in Associahedra, Tamari Lattices and Related Structures, Prog. Math. Phys., Vol. 299, Editors F. Müller-Hoissen, J. Pallo, J. Stasheff, Birkhäuser/Springer, Basel, 2012, 391-423, arXiv:1110.3507.

[26] Doliwa A., Sergeev S.M., The pentagon relation and incidence geometry, arXiv:1208.3339.

[27] Drinfeld V.G., Quasi-Hopf algebras, Leningrad Math. J. 1 (1989), 1419-1457.

[28] Drinfeld V.G., On quasitriangular quasi-Hopf algebras and on a group that is closely connected with $\operatorname{Gal}(\overline{\mathbf{Q}} / \mathbf{Q})$, Leningrad Math. J. 2 (1990), 829-860.

[29] Drinfeld V.G., On some unsolved problems in quantum group theory, in Quantum Groups (Leningrad, 1990), Lecture Notes in Math., Vol. 1510, Editor P.P. Kulish, Springer, Berlin, 1992, 1-8.

[30] Edelman P.H., Reiner V., The higher Stasheff-Tamari posets, Mathematika 43 (1996), 127-154.

[31] Elias B., Williamson G., Soergel calculus, arXiv:1309.0865.

[32] Etingof P., Geometric crystals and set-theoretical solutions to the quantum Yang-Baxter equation, Comm. Algebra 31 (2003), 1961-1973, math.QA/0112278.

[33] Etingof P., Kazhdan D., Quantization of Lie bialgebras. I, Selecta Math. (N.S.) 2 (1996), 1-41, q-alg/9506005.

[34] Etingof P., Latour F., The dynamical Yang-Baxter equation, representation theory, and quantum integrable systems, Oxford Lecture Series in Mathematics and its Applications, Vol. 29, Oxford University Press, Oxford, 2005. 
[35] Etingof P., Schedler T., Soloviev A., Set-theoretical solutions to the quantum Yang-Baxter equation, Duke Math. J. 100 (1999), 169-209, math.QA/9801047.

[36] Etingof P., Schiffmann O., Lectures on quantum groups, Lectures in Mathematical Physics, International Press, Boston, MA, 1998.

[37] Faddeev L.D., Kashaev R.M., Quantum dilogarithm, Modern Phys. Lett. A 9 (1994), 427-434.

[38] Felsner S., Ziegler G.M., Zonotopes associated with higher Bruhat orders, Discrete Math. 241 (2001), 301312 .

[39] Frenkel I., Moore G., Simplex equations and their solutions, Comm. Math. Phys. 138 (1991), 259-271.

[40] Furusho H., Pentagon and hexagon equations, Ann. of Math. 171 (2010), 545-556, math.QA/0702128.

[41] Furusho H., Around associators, in Automorphic Forms and Galois Representations, London Mathematical Society Lecture Note Series, Vol. 415, Editors M. Kim, F. Diamond, P.L. Kassaei, Cambridge University Press, Cambridge, 2014, 105-117.

[42] Goncharenko V.M., Veselov A.P., Yang-Baxter maps and matrix solitons, in New Trends in Integrability and Partial Solvability, NATO Science Series II: Math. Phys. Chem., Vol. 132, Editors A.B. Shabat, A. González-López, M. Mañas, L. Martínez Alonso, M.A. Rodríguez, Kluwer, Dordrecht, 2004, 191-197, math-ph/0303032.

[43] Hietarinta J., Labelling schemes for tetrahedron equations and dualities between them, J. Phys. A: Math. Gen. 27 (1994), 5727-5748, hep-th/9402139.

[44] Hietarinta J., Nijhoff F., The eight tetrahedron equations, J. Math. Phys. 38 (1997), 3603-3615, q-alg/9706001.

[45] Ip I.C.-H., Representation of the quantum plane, its quantum double, and harmonic analysis on $G L_{q}^{+}(2, \mathbb{R})$, Selecta Math. (N.S.) 19 (2013), 987-1082, arXiv:1108.5365.

[46] Izumi M., Kosaki H., Kac algebras arising from composition of subfactors: general theory and classification, Mem. Amer. Math. Soc. 158 (2002), no. 750, 198 pages.

[47] Jimbo M., Introduction to the Yang-Baxter equation, Internat. J. Modern Phys. A 4 (1989), 3759-3777.

[48] Jimbo M. (Editor), Yang-Baxter equation in integrable systems, Advanced Series in Mathematical Physics, Vol. 10, World Scientific Publishing Co. Inc., Singapore, 1990.

[49] Kapranov M.M., Voevodsky V.A., Combinatorial-geometric aspects of polycategory theory: pasting schemes and higher Bruhat orders (list of results), Cahiers Topologie Géom. Différentielle Catég. 32 (1991), 11-27.

[50] Kapranov M.M., Voevodsky V.A., 2-categories and Zamolodchikov tetrahedra equations, in Algebraic Groups and their Generalizations: Quantum and Infinite-Dimensional Methods (University Park, PA, 1991), Proc. Sympos. Pure Math., Vol. 56, Amer. Math. Soc., Providence, RI, 1994, 177-259.

[51] Kapranov M.M., Voevodsky V.A., Braided monoidal 2-categories and Manin-Schechtman higher braid groups, J. Pure Appl. Algebra 92 (1994), 241-267.

[52] Kashaev R.M., The Heisenberg double and the pentagon relation, St. Petersbg. Math. J. 8 (1997), 585-592, q-alg/9503005.

[53] Kashaev R.M., On matrix generalizations of the dilogarithm, Theoret. and Math. Phys. 118 (1999), 314-318.

[54] Kashaev R.M., A simple model of 4D-TQFT, arXiv:1405.5763.

[55] Kashaev R.M., Nakanishi T., Classical and quantum dilogarithm identities, SIGMA 7 (2011), 102, 29 pages, arXiv:1104.4630.

[56] Kashaev R.M., Reshetikhin N., Symmetrically factorizable groups and self-theoretical solutions of the pentagon equation, in Quantum Groups, Contemp. Math., Vol. 433, Amer. Math. Soc., Providence, RI, 2007, 267-279, math.QA/0111171.

[57] Kashaev R.M., Sergeev S.M., On pentagon, ten-term, and tetrahedron relations, Comm. Math. Phys. 195 (1998), 309-319, q-alg/9607032.

[58] Kazhdan D., Soibelman Y., Representations of the quantized function algebras, 2-categories and Zamolodchikov tetrahedra equation, in The Gel'fand Mathematical Seminars, 1990-1992, Birkhäuser Boston, Boston, MA, 1993, 163-171.

[59] Korepanov I.G., Tetrahedral Zamolodchikov algebras corresponding to Baxter's L-operators, Comm. Math. Phys. 154 (1993), 85-97.

[60] Korepanov I.G., SL(2)-solution of the pentagon equation and invariants of three-dimensional manifolds, Theoret. and Math. Phys. 138 (2004), 18-27, math.AG/0304149. 
[61] Korepanov I.G., Relations in Grassmann algebra corresponding to three- and four-dimensional Pachner moves, SIGMA 7 (2011), 117, 23 pages, arXiv:1105.0782.

[62] Korepanov I.G., Deformation of a $3 \rightarrow 3$ Pachner move relation capturing exotic second homologies, arXiv:1201.4762.

[63] Korepanov I.G., Special 2-cocycles and 3-3 Pachner move relations in Grassmann algebra, arXiv:1301.5581.

[64] Korepanov I.G., Two-cocycles give a full nonlinear parameterization of the simplest 3-3 relation, Lett. Math. Phys. 104 (2014), 1235-1261, arXiv:1310.4075.

[65] Korepanov I.G., Multiplicative expression for the coefficient in fermionic 3-3 relation, arXiv:1503.02272.

[66] Korepanov I.G., Sadykov N.M., Parameterizing the simplest Grassmann-Gaussian relations for Pachner move 3-3, SIGMA 9 (2013), 053, 19 pages, arXiv:1305.3246.

[67] Korepanov I.G., Saĭto S., Finite-dimensional analogues of the string $s \leftrightarrow t$ duality, and the pentagon equation, Theoret. and Math. Phys. 120 (1999), 862-869.

[68] Korepanov I.G., Sharygin G.I., Talalaev D.V., Cohomologies of $n$-simplex relations, arXiv:1409.3127.

[69] Kulish P.P., Representation of the Zamolodchikov-Faddeev algebra, J. Sov. Math. 24 (1984), $208-215$.

[70] Lawrence R.J., Algebras and triangle relations, J. Pure Appl. Algebra 100 (1995), 43-72.

[71] Mac Lane S., Natural associativity and commutativity, Rice Univ. Studies 49 (1963), 28-46.

[72] Maillet J.-M., On pentagon and tetrahedron equations, St. Petersburg Math. J. 6 (1995), 375-383, hep-th/9312037.

[73] Maillet J.-M., Nijhoff F., Multidimensional integrable lattice models, quantum groups, and the $d$-simplex equations, Report CERN.TH-5595/89, 1989.

[74] Maillet J.-M., Nijhoff F., Integrability for multidimensional lattice models, Phys. Lett. B 224 (1989), 389396.

[75] Maillet J.-M., Nijhoff F., On the algebraic structure of integrable systems in multidimensions, in XVIIth International Colloquium on Group Theoretical Methods in Physics (Sainte-Adèle, PQ, 1988), Editors Y. Saint-Aubin, L. Vinet, World Sci. Publ., Teaneck, NJ, 1989, 504-507.

[76] Maillet J.-M., Nijhoff F., The tetrahedron equation and the four-simplex equation, Phys. Lett. A 134 (1989), 221-228.

[77] Maillet J.-M., Nijhoff F., Multidimensional lattice integrability and the simplex equations, in Nonlinear Evolution Equations: Integrability and Spectral Methods, Editors A. Degasperis, A.P. Fordy, M. Lakshmanan, Manchester University Press, Manchester, 1990, 537-548.

[78] Manin Yu.I., Schechtman V.V., Arrangements of real hyperplanes and Zamolodchikov equations, in Group Theoretical Methods in Physics, Vol. I (Yurmala, 1985), Editors M.A. Markov, V.I. Man'ko, V.V. Dodonov, VNU Sci. Press, Utrecht, 1986, 151-165.

[79] Manin Yu.I., Shechtman V.V., Higher Bruhat orders, related to the symmetric group, Funct. Anal. Appl. 20 (1986), 148-150.

[80] Manin Yu.I., Schechtman V.V., Arrangements of hyperplanes, higher braid groups and higher Bruhat orders, in Algebraic Number Theory - in Honor of K. Iwasawa, Adv. Stud. Pure Math., Vol. 17, Editors J. Coates, R. Greenberg, B. Mazur, I. Satake, Academic Press, Boston, MA, 1989, 289-308.

[81] Masuda T., Nakagami Y., A von Neumann algebra framework for the duality of the quantum groups, Publ. Res. Inst. Math. Sci. 30 (1994), 799-850.

[82] Michielsen F.P., Nijhoff F.W., $d$-algebras, the $d$-simplex equations, and multidimensional integrability, in Quantum Topology, Ser. Knots Everything, Vol. 3, Editors L.H. Kauffman, R.A. Baadhio, World Sci. Publ., River Edge, NJ, 1993, 230-243.

[83] Militaru G., Heisenberg double, pentagon equation, structure and classification of finite-dimensional Hopf algebras, J. London Math. Soc. 69 (2004), 44-64.

[84] Moore G., Seiberg N., Classical and quantum conformal field theory, Comm. Math. Phys. 123 (1989), 177-254.

[85] Müller-Hoissen F., Pallo J.M., Stasheff J. (Editors), Associahedra, Tamari lattices and related structures, Prog. Math. Phys., Vol. 299, Birkhäuser/Springer, Basel, 2012.

[86] Rambau J., Reiner V., A survey of the higher Stasheff-Tamari orders, in Associahedra, Tamari Lattices and Related Structures, Prog. Math. Phys., Vol. 299, Editors F. Müller-Hoissen, J.M. Pallo, J. Stasheff, Birkhäuser/Springer, Basel, 2012, 351-390. 
[87] Rogers L.J., On function sum theorems connected with the series formula, Proc. London Math. Soc. S2-4 (1907), 169-189.

[88] Sergeev S.M., On a two dimensional system associated with the complex of the solutions of the tetrahedron equation, solv-int/9709013.

[89] Sergeev S.M., Mathematics of quantum integrable systems in multidimensional discrete space-time, Preliminary draft, version 4, 2009, available at http://ise.canberra.edu.au/mathphysics/files/2009/09/ book5.pdf.

[90] Skandalis G., Operator algebras and duality, in Proceedings of the International Congress of Mathematicians, Vols. I, II (Kyoto, 1990), Math. Soc. Japan, Tokyo, 1991, 997-1009.

[91] Sklyanin E.K., Classical limits of SU(2)-invariant solutions of the Yang-Baxter equation, J. Sov. Math. 40 (1988), 93-107.

[92] Stasheff J.D., Homotopy associativity of H-spaces. I, Trans. Amer. Math. Soc. 108 (1963), 275-292.

[93] Street R., Higher categories, strings, cubes and simplex equations, Appl. Categ. Structures 3 (1995), 29-77.

[94] Street R., Fusion operators and cocycloids in monoidal categories, Appl. Categ. Structures 6 (1998), $177-191$.

[95] Stroganov Yu.G., The tetrahedron equation and spin integrable models on the cubic lattice, Theoret. and Math. Phys. 110 (1997), 141-167.

[96] Suris Yu.B., Veselov A.P., Lax matrices for Yang-Baxter maps, J. Nonlinear Math. Phys. 10 (2003), suppl. 2, 223-230, math.QA/0304122.

[97] Tamari D., Monoides préordonnés et chaînes de Malcev, Ph.D. Thesis, Paris, 1951.

[98] Veselov A.P., Yang-Baxter maps and integrable dynamics, Phys. Lett. A 314 (2003), 214-221, math.QA/0205335.

[99] Veselov A.P., Yang-Baxter maps: dynamical point of view, in Combinatorial Aspect of Integrable Systems, MSJ Mem., Vol. 17, Editors A. Berenstein, D. Kazhdan, C. Lecouvey, M. Okado, A. Schilling, T. Takagi, A. Veselov, Math. Soc. Japan, Tokyo, 2007, 145-167, math.QA/0612814.

[100] Volkov A.Yu., Beyond the "pentagon identity", Lett. Math. Phys. 39 (1997), 393-397, q-alg/9603003.

[101] Woronowicz S.L., From multiplicative unitaries to quantum groups, Internat. J. Math. 7 (1996), $127-149$.

[102] Woronowicz S.L., Zakrzewski S., Quantum ' $a x+b$ ' group, Rev. Math. Phys. 14 (2002), 797-828.

[103] Yang C.N., Some exact results for the many-body problem in one dimension with repulsive delta-function interaction, Phys. Rev. Lett. 19 (1967), 1312-1315.

[104] Zakrzewski S., Poisson Lie groups and pentagonal transformations, Lett. Math. Phys. 24 (1992), 13-19.

[105] Zamolodchikov A.B., Tetrahedra equations and integrable systems in three-dimensional space, Sov. Phys. JETP 52 (1980), 325-336.

[106] Zamolodchikov A.B., Tetrahedron equations and the relativistic $S$-matrix of straight-strings in $2+1-$ dimensions, Comm. Math. Phys. 79 (1981), 489-505.

[107] Ziegler G.M., Higher Bruhat orders and cyclic hyperplane arrangements, Topology 32 (1993), $259-279$. 